\title{
Search for electroweak production of a vector-like $T$ quark using fully hadronic final states
}

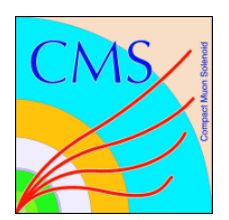

\section{The CMS collaboration}

E-mail: cms-publication-committee-chair@cern.ch

ABSTRACT: A search is performed for electroweak production of a vector-like top quark partner $\mathrm{T}$ of charge $2 / 3$ in association with a top or bottom quark, using proton-proton collision data at $\sqrt{s}=13 \mathrm{TeV}$ collected by the CMS experiment at the LHC in 2016. The data sample corresponds to an integrated luminosity of $35.9 \mathrm{fb}^{-1}$. The search targets $\mathrm{T}$ quarks over a wide range of masses and fractional widths, decaying to a top quark and either a Higgs boson or a Z boson in fully hadronic final states. The search is performed using two experimentally distinct signatures that depend on whether or not each quark from the decays of the top quark, Higgs boson, or $\mathrm{Z}$ boson produces an individual resolved jet. Jet substructure, $b$ tagging, and kinematic variables are used to identify the top quark and boson jets, and also to suppress the standard model backgrounds. The data are found to be consistent with the expected backgrounds. Upper limits at $95 \%$ confidence level are set on the cross sections for T quark-mediated production of tHQq, tZQq, and their sum, where $\mathrm{Q}$ is the associated top or bottom heavy quark and $\mathrm{q}$ is another associated quark. The limits are given for each search signature for various T quark widths up to $30 \%$ of the T quark mass, and are between $2 \mathrm{pb}$ and $20 \mathrm{fb}$ for T quark masses in the range $0.6-2.6 \mathrm{TeV}$. These results are significantly more sensitive than prior searches for electroweak single production of $\mathrm{T} \rightarrow \mathrm{tH}$ and represent the first constraints on $\mathrm{T} \rightarrow \mathrm{tZ}$ using hadronic decays of the $\mathrm{Z}$ boson with this production mode.

KEYwORDS: Hadron-Hadron scattering (experiments), vector-like quarks

ARXIV EPRINT: 1909.04721 


\section{Contents}

1 Introduction 1

2 The CMS detector and event reconstruction 4

$\begin{array}{lll}3 & \text { Data and modeling of signals and backgrounds } & 6\end{array}$

4 Reconstruction methods and primary selection $\quad 7$

5 Low-mass search $\quad 8$

5.1 Event selection 8

$\begin{array}{lll}5.2 & \text { Background estimation and validation } & 11\end{array}$

5.3 Low-mass search results 12

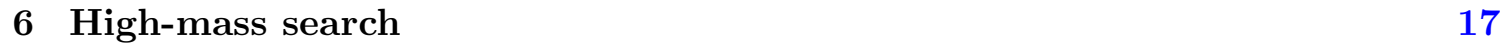

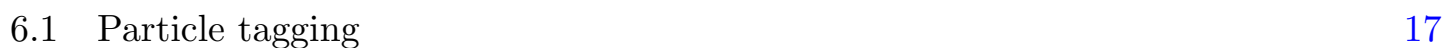

$\begin{array}{lll}6.2 & \text { Event selection } & 18\end{array}$

$\begin{array}{lll}6.3 & \text { Background estimation } & 19\end{array}$

6.4 High-mass search results 23

7 Systematic uncertainties $\quad 24$

$\begin{array}{llr}8 & \text { Search results } & 28\end{array}$

$\begin{array}{llr}9 & \text { Summary } & 29\end{array}$

A Low-mass and high-mass search limits $\quad 36$

The CMS collaboration $\quad 44$

\section{Introduction}

We report on a search for electroweak production of a new heavy quark of charge $2 / 3$ with nonchiral couplings, referred to as a vector-like quark. Unlike the standard model (SM) chiral fermions, such particles do not acquire their mass from a Yukawa coupling to the Higgs boson (H). Many proposed extensions of the SM contain vector-like quarks, which usually mix with the top quark (t). Such particles could have a role in stabilizing the Higgs boson mass, and thus offer a potential solution to the hierarchy problem. Vector-like quarks are discussed in detail in refs. [1-4] and have been the subject of phenomenological studies in various frameworks including those of refs. [5-8]. 

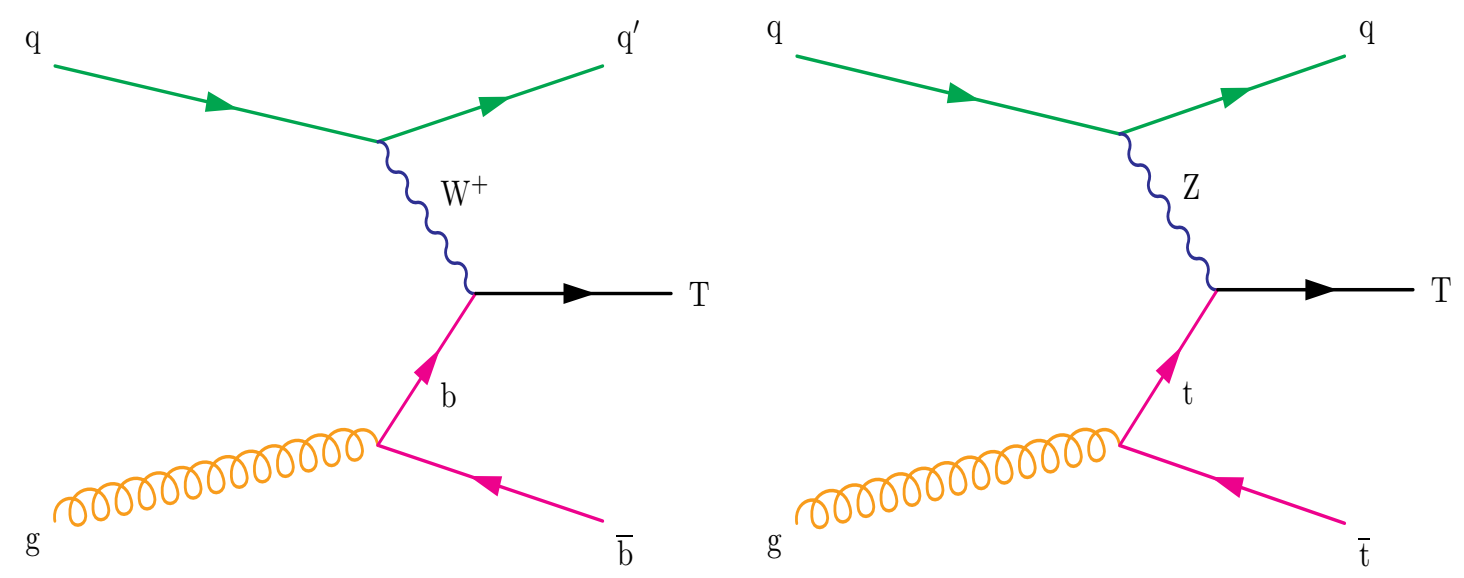

Figure 1. Example Feynman diagrams for electroweak production of vector-like $\mathrm{T}$ quarks. Charged-current (left) and neutral current (right).

Much like the top quark, a vector-like top quark partner (T) could be produced either in pairs, dominantly through the strong interaction, or singly, in association with additional quarks through the electroweak interaction. The $\mathrm{T}$ quark could couple to $\mathrm{bW}$, tZ, or $\mathrm{tH}$; this leads to the corresponding $\mathrm{T}$ quark decays and to the associated electroweak production from processes such as those depicted in figure 1. The branching fractions and dominant electroweak production processes depend on the particular model; many models have substantial branching fractions to $\mathrm{tZ}$ or $\mathrm{tH}$ resulting in signatures that are of primary relevance to this paper. Neglecting the corrections due to decay particle masses, the branching fractions for the T singlet model of ref. [5] are $50 \%$ (bW), $25 \%(\mathrm{tZ}), 25 \%$ $(\mathrm{tH})$, while for the (TB) doublet model of ref. [5], the $\mathrm{tZ}$ and $\mathrm{tH}$ branching fractions tend to be approximately equal and depend on two mixing angles, $\theta_{R}^{u}$ and $\theta_{R}^{d}$, with each branching fraction ranging from zero to $50 \%$. Therefore specific models can have branching fractions as large as $50 \%$ for $\mathrm{tZ}$ and $50 \%$ for $\mathrm{tH}$.

We perform a search targeting the electroweak production of a vector-like top quark partner $\mathrm{T}$ in fully hadronic final states in proton-proton (pp) collisions at $\sqrt{s}=13 \mathrm{TeV}$ with the CMS detector at the CERN LHC. We use two searches that target separately lower and higher mass values for the $\mathrm{T}$ quark. Both searches are designed to be sensitive to the decay to a top quark and a Higgs boson $(\mathrm{T} \rightarrow \mathrm{tH})$, and to the decay to a top quark and a $\mathrm{Z}$ boson $(\mathrm{T} \rightarrow \mathrm{tZ})$ with subsequent hadronic decays of $\mathrm{X}(\mathrm{X}=\mathrm{H}, \mathrm{Z})$. Both also consider a wide range of widths of the $\mathrm{T}$ quark, ranging from narrow, defined as small compared to the experimental mass resolution, to as much as $30 \%$ of the $\mathrm{T}$ quark mass. The event selections primarily require $\mathrm{b}$ tagging for the Higgs and $\mathrm{Z}$ boson candidates and so are most sensitive to $X \rightarrow b \bar{b}$. The experimental signature is a resonant peak in the $t X$ invariant mass spectrum.

The searches are designed to seek evidence of $\mathrm{T}$ quarks produced in association with a bottom quark, dominated by the $\mathrm{qg} \rightarrow \mathrm{T} \overline{\mathrm{b}} \mathrm{q}^{\prime}$ process, and, separately, associated production with a top quark dominated by the qg $\rightarrow T \overline{\mathrm{t}} \mathrm{q}$ process, where the charge conjugate processes are also implied. These are electroweak production modes, with the production of 
only a single $\mathrm{T}$, that rely on a nonzero TbW coupling for the charged-current production, and a nonzero TtZ coupling for the neutral-current production. In order to be produced with an observable cross section, one needs a substantial partial width for the coupling to the initial state. As a consequence, currently accessible production cross sections in electroweak single production are associated with particle widths exceeding about $5 \%$, which would affect the experimentally observable invariant mass distributions. The total width could also be enlarged if additional decay modes were present.

As a result of the lower requirement on the constituent center-of-mass energy and the larger available phase space, single production via the electroweak mechanism allows a search for vector-like top quarks with masses beyond those already tested with pair production. The $\mathrm{qg} \rightarrow \mathrm{T} \overline{\mathrm{b}} \mathrm{q}^{\prime} \rightarrow \mathrm{tX} \overline{\mathrm{b}} \mathrm{q}^{\prime}$ process, with the top quark from the $\mathrm{T}$ quark decaying hadronically and $\mathrm{X}$ decaying to two $\mathrm{b}$ quarks, results in up to seven jets, four of which are $\mathrm{b}$ jets. The seven jets are associated with the production of seven fermions, namely qg $\rightarrow\left(\mathrm{q}_{\overline{\mathrm{q}}}^{\prime} \mathrm{b}\right)(\mathrm{b} \overline{\mathrm{b}}) \overline{\mathrm{b}} \mathrm{q}^{\prime}$. Similarly the $\mathrm{qg} \rightarrow \mathrm{T} \overline{\mathrm{t}} \mathrm{q} \rightarrow \mathrm{tX} \overline{\mathrm{t}} \mathrm{q}$ process results in at least nine fermions. In each case the other associated quark ( $\mathrm{q}^{\prime}$ or $\mathrm{q}$ ) often results in a forward jet at high absolute pseudorapidity. The $\mathrm{qg} \rightarrow \mathrm{T} \overline{\mathrm{b}} \mathrm{q}^{\prime}$ process is expected to have a higher cross section than $\mathrm{qg} \rightarrow \mathrm{T} \overline{\mathrm{tq}}$ from kinematic and coupling considerations.

Recent searches at the LHC for pair production of vector-like quarks have severely constrained the possible existence of lower-mass vector-like quarks that couple to heavy quarks [9-18]. These searches use several final states arising from the bW, tZ, and tH decay channels and usually model pair production under the assumption of a narrow width. In particular, for the $\mathrm{T}$ singlet model, the most stringent expected lower mass limit from pair production to date is $1.2 \mathrm{TeV}$ [16] at 95\% confidence level (CL). Pair production is based on the assumed universal strong coupling and so the quantum chromodynamics (QCD) pair production cross sections are known and model independent, and depend only on the $\mathrm{T}$ quark mass, $m_{\mathrm{T}}$. On the other hand, electroweak production depends on the strength of the $\mathrm{T}$ quark coupling at the production vertex, either $\mathrm{TbW}$ or $\mathrm{TtZ}$, and therefore the production cross sections are model dependent. In some models, such as that of ref. [2], the couplings are constrained by precision observables to be quite small. In other models, such as that of ref. [7], cross sections two orders of magnitude higher than in ref. [2] may be feasible. The first direct experimental constraints on electroweak production of vector-like quarks were published in ref. [19]. Search results at $\sqrt{s}=13 \mathrm{TeV}$ include the search already performed by CMS for electroweak production of $\mathrm{T}$ with $\mathrm{T} \rightarrow \mathrm{tH}$ for both semileptonically decaying top quarks and hadronically decaying top quarks using the 2015 data set $[20,21]$. Other results at $\sqrt{s}=13 \mathrm{TeV}$ targeting electroweak production of $\mathrm{T}$ are described in refs. $[22,23]$ for $\mathrm{T} \rightarrow \mathrm{bW}$, and for $\mathrm{T} \rightarrow \mathrm{tZ}$ with dielectron and dimuon decays of the $\mathrm{Z}$ in refs. [15, 24, 25], and using a missing energy signature for $\mathrm{Z} \rightarrow \nu \bar{\nu}$ decays in ref. [26]. The search reported here uses the 2016 data set to study fully hadronic final states with both merged and resolved jets resulting from electroweak production of a $\mathrm{T}$ quark with $\mathrm{T} \rightarrow \mathrm{tH}$ and $\mathrm{T} \rightarrow \mathrm{tZ}$. This search represents a significant advance over prior electroweak production searches for $\mathrm{T} \rightarrow \mathrm{tH}$, with expected 95\% CL cross section upper limits typically $5-10$ times lower than those reported in ref. [21], and is competitive with other searches for $\mathrm{T} \rightarrow \mathrm{tZ}$ in this production mode. 
For low values of the $\mathrm{T}$ quark mass, the quarks resulting from the top quark decay and from the Higgs or Z boson decay can be resolved as individual jets. However, as the $\mathrm{T}$ quark mass increases, the larger Lorentz boost from the decay will lead to the decay products of the top quark and the quarks from the Higgs or $\mathrm{Z}$ boson becoming progressively less and less resolved as separate jets. The jet multiplicity, correspondingly, is reduced. Furthermore, one can reconstruct both the top quark and the Higgs or Z boson by using large-area jet and substructure techniques, where area refers to the jet's extent in $\eta-\phi$ space. Consequently, two search signatures are defined as follows:

- Low-mass search: reconstruction of a five-jet invariant mass signature for the $\mathrm{T} \rightarrow \mathrm{tH}$ and $\mathrm{T} \rightarrow \mathrm{tZ}$ decay modes. This search signature is based on multijet triggers with $\mathrm{b}$ tagging and is effective for low $\mathrm{T}$ masses $(0.6-1.2 \mathrm{TeV})$ where the individual jets from the decays can be resolved.

- High-mass search: reconstruction of an invariant mass signature from two large-area jets for both the $\mathrm{T} \rightarrow \mathrm{tH}$ and $\mathrm{T} \rightarrow \mathrm{tZ}$ decay modes. This search signature is based on triggers using high transverse momentum jets and is effective for high $\mathrm{T}$ mass $(>1.0 \mathrm{TeV})$. In this mass range, the final state particles from the decays of each of the two daughter particles resulting from the $\mathrm{T}$ quark decay (the $\mathrm{t}$ and the $\mathrm{H}$ or $\mathrm{Z}$ ) produce a single large-area jet. This leads to events with two large-area jets.

Each search is designed to be sensitive to $\mathrm{T}$ quark production in association with either a bottom quark or a top quark. Besides the primary motivation of exploring the possibility of electroweak production of a vector-like quark, this analysis can be viewed more broadly as two independent searches for high mass signatures of physics beyond the SM at the LHC. As such they provide potential for discovery of new physics, independent of the specific models discussed here.

The paper is organized as follows: this section has given the motivation to search for the singly produced $\mathrm{T}$ quark with two distinct signatures and two decay modes. The CMS detector and event reconstruction are described in section 2. The data set and the modeling of signal and background processes are described in section 3. Reconstruction methods common to the two searches are discussed in section 4 . The event selection criteria, background estimation, and results are described for the low-mass search in section 5 , and for the high-mass search in section 6 . Systematic uncertainties for both signatures are discussed in section 7 . The overall results are presented in section 8 and summarized in section 9 .

\section{The CMS detector and event reconstruction}

The central feature of the CMS apparatus is a superconducting solenoid of $6 \mathrm{~m}$ internal diameter, providing a magnetic field of $3.8 \mathrm{~T}$. Within the solenoid volume are a silicon pixel and strip tracker, a lead tungstate crystal electromagnetic calorimeter (ECAL), and a brass and scintillator hadron calorimeter (HCAL), each composed of a barrel and two endcap sections. Forward calorimeters extend the pseudorapidity coverage provided by the 
barrel and endcap detectors. Muons are detected in gas-ionization chambers embedded in the steel flux-return yoke outside the solenoid.

The silicon tracker measures charged particles within the pseudorapidity range $|\eta|<$ 2.5. For nonisolated particles with transverse momentum, $p_{\mathrm{T}}$, in the range $1<p_{\mathrm{T}}<10 \mathrm{GeV}$ and $|\eta|<1.4$, the track resolutions are typically $1.5 \%$ in $p_{\mathrm{T}}$ and $25-90(45-150) \mu \mathrm{m}$ in the transverse (longitudinal) impact parameter [27].

The ECAL consists of 75848 crystals covering $|\eta|<3.00$. The HCAL cells have widths of 0.087 in pseudorapidity and 0.087 in azimuth $(\phi)$ in the region $|\eta|<1.74$. In the $\eta$ - $\phi$ plane, and for $|\eta|<1.48$, the HCAL cells map onto $5 \times 5$ arrays of ECAL crystals to form calorimeter towers projecting radially outwards from close to the nominal interaction point. For $|\eta|>1.74$, the coverage of the towers increases progressively with $|\eta|$ to a maximum of 0.174 in $\Delta \eta$ and $\Delta \phi$. The forward calorimeters extend the calorimetric coverage for hadronic jets to $|\eta|=5.0$.

Events of interest are selected using a two-tiered trigger system [28]. The first level, composed of custom hardware processors, uses information from the calorimeters and muon detectors to select events at a rate of around $100 \mathrm{kHz}$ within a time interval of less than $4 \mu \mathrm{s}$. The second level, known as the high-level trigger, consists of a farm of processors running a version of the full event reconstruction software optimized for fast processing, and reduces the event rate to around $1 \mathrm{kHz}$ before data storage.

In the reconstruction, the vertex with the largest value of summed physics-object $p_{\mathrm{T}}^{2}$ is taken to be the primary pp interaction vertex. The physics objects are the jets, clustered using the anti- $k_{\mathrm{T}}$ jet finding algorithm [29, 30], with the tracks assigned to the vertex as inputs.

A particle-flow algorithm [31] aims to reconstruct and identify each individual particle in an event, with an optimized combination of information from the various elements of the CMS detector. The energy of photons is directly obtained from the ECAL measurement, corrected for zero-suppression effects. The energy of electrons is determined from a combination of the electron momentum at the primary interaction vertex as determined by the tracker, the energy of the corresponding ECAL cluster, and the energy sum of all bremsstrahlung photons spatially compatible with originating from the electron track. The momentum of muons is obtained from the fitted trajectory of the corresponding track reconstructed from the tracker and the muon detectors. The energy of charged hadrons is determined from a combination of their momentum measured in the tracker and the matching ECAL and HCAL energy deposits, corrected for zero-suppression effects and for the response function of the calorimeters to hadronic showers. Finally, the energy of neutral hadrons is obtained from the corresponding corrected ECAL and HCAL energies.

Jet momentum is determined as the vectorial sum of all particle momenta in the jet, and is found from simulation to be within 5 to $10 \%$ of the true momentum over the whole $p_{\mathrm{T}}$ spectrum and detector acceptance. Additional pp interactions within the same or nearby bunch crossings (pileup) can contribute additional tracks and calorimetric energy depositions to the jet momentum. To mitigate this effect, tracks identified to be originating from pileup vertices are discarded, and an offset correction is applied to correct for remaining contributions. Jet energy corrections are derived from simulation 
to bring the measured response of jets to that of particle-level jets on average. In situ measurements of the momentum balance in dijet, photon + jet, $\mathrm{Z}+$ jet, and multijet events are used to estimate any residual differences in jet energy scale in data and simulation [32]. Additional selection criteria are applied to each jet to remove jets potentially dominated by anomalous contributions from various subdetector components or reconstruction failures. The jet energy resolution amounts typically to $15 \%$ at $10 \mathrm{GeV}, 8 \%$ at $100 \mathrm{GeV}$, and $4 \%$ at $1 \mathrm{TeV}$, to be compared to about 40,12 , and $5 \%$ obtained when the calorimeters alone are used for jet clustering [31].

A more detailed description of the CMS detector, together with a definition of the coordinate system used and the relevant kinematic variables, can be found in ref. [33].

\section{Data and modeling of signals and backgrounds}

This analysis uses proton-proton collision data collected at a center-of-mass energy of $\sqrt{s}=13 \mathrm{TeV}$, recorded in 2016, amounting to a total integrated luminosity of $35.9 \mathrm{fb}^{-1}$.

Simulated samples for the $2 \rightarrow 3$ signal processes, $\mathrm{pp} \rightarrow \mathrm{Tbq}$ and $\mathrm{pp} \rightarrow \mathrm{Ttq}$, were generated at leading order using the Monte Carlo (MC) event generator MADGRAPH5_aMC@NLO 2.3.3 [34] for various masses of the T quark and for the decays T $\rightarrow$ tH and $\mathrm{T} \rightarrow \mathrm{tZ}$. The signal generation for these "narrow width" $2 \rightarrow 3$ process samples has the width set to $10 \mathrm{GeV}$, which is small on the scale of the experimental resolution of about $6 \%$. Separate samples were generated for both left- and right-handed chiralities of the T quark for each decay mode, for these narrow-width cases. In addition, MADGRAPH5_aMC@NLO 2.4.2 at leading order was used to simulate the large width $2 \rightarrow 4$ processes, $\mathrm{pp} \rightarrow \mathrm{tHbq}$, $\mathrm{pp} \rightarrow \mathrm{tHtq}, \mathrm{pp} \rightarrow \mathrm{tZbq}$ and $\mathrm{pp} \rightarrow \mathrm{tZtq}$, with fractional widths $\Gamma / m_{\mathrm{T}}$ of 10,20 , and $30 \%$. All of the large-width samples assume left- (right-)handed $\mathrm{T}$ chiralities for the $\mathrm{pp} \rightarrow \mathrm{Tbq}$ $(\mathrm{pp} \rightarrow \mathrm{Ttq})$ case, as expected in the singlet (doublet) model.

The benchmark $\mathrm{T}$ quark masses used for the results range from 0.6 to $2.6 \mathrm{TeV}$. The NNPDF3.0 parton distribution function (PDF) set [35] was used. The samples are generated with both the top quark and the Higgs boson decaying inclusively. The masses of the Higgs boson and top quark are set to 125 and $172.5 \mathrm{GeV}$, respectively. In the samples, the SM Higgs boson branching fraction $\mathcal{B}(\mathrm{H} \rightarrow \mathrm{b} \overline{\mathrm{b}})$ of $58 \%$ is assumed. Similarly the $\mathrm{t}$ and $\mathrm{Z}$ are decayed inclusively in the $\mathrm{T} \rightarrow \mathrm{tZ}$ samples.

The SM background simulation samples include $t \overline{\mathrm{t}}+$ jets, $W+$ jets, $Z+$ jets, single top quark, $\mathrm{tHq}, \mathrm{t} \overline{\mathrm{t}} \mathrm{H}, \mathrm{t} \overline{\mathrm{t}} \mathrm{W}, \mathrm{t} \overline{\mathrm{t} Z}, \mathrm{WW}, \mathrm{ZZ}, \mathrm{WZ}, \mathrm{WH}$, and $\mathrm{ZH}$. These processes are generated at next-to-leading order with MADGRAPH5_aMC@NLO 2.3.3 unless otherwise specified. The parton-level MC simulations for SM backgrounds and signal are interfaced with PYTHiA 8.212 [36]. The large-width signal samples and the main backgrounds involving top quarks use the CUETP8M2T4 tune [37]. The other samples use the earlier CUETP8M1 tune [38].

The $t \bar{t}+$ jets events are inclusive, and are simulated using POWHEG 2.0 [39-42]. The $\mathrm{W}+$ jets and $\mathrm{Z}+$ jets samples include only hadronic $\mathrm{W}$ or $\mathrm{Z}$ boson decays and contain an $H_{\mathrm{T}}>600 \mathrm{GeV}$ requirement, where $H_{\mathrm{T}}$ is the scalar sum of jet transverse momenta. The single top quark tW process was generated using the POWHEG 2.0+PYTHIA 8 generator 
combination. The SM tHq process simulation included all decay modes for the top quark and Higgs boson. The tet $\mathrm{H}$ sample was generated with the decay $\mathrm{H} \rightarrow \mathrm{b} \overline{\mathrm{b}}$ with a Higgs boson mass of $125 \mathrm{GeV}$ using POWHEG 2.0. The WW sample was generated with hadronic decays using POWHEG 2.0. The ZZ sample was generated with hadronic decays using MADSPIN [43] and applying the FxFx merging procedure [44] for matching jets from the matrix element calculation with those from the parton shower. The WZ sample was inclusive and generated with PYTHIA 8. The $\mathrm{ZH}$ sample was generated with $\mathrm{Z} \rightarrow \mathrm{b} \overline{\mathrm{b}}$ with POWHEG 2.0.

The SM background events comprised uniquely of jets produced through the strong interaction, referred to as QCD multijet events, were also considered in the design of the analyses. Two sets of simulated samples were used: a sample generated using PYTHIA 8 that is binned in the invariant $p_{\mathrm{T}}$ associated with the hard process and an $H_{\mathrm{T}}$-binned sample using MADGRAPH5_aMC@NLO at leading order with up to four partons in the matrix element calculations, using the MLM jet matching scheme [45] with PYTHIA fragmentation and showering. All simulated event samples were generated using the NNPDF3.0 PDF set except that based on PYTHIA 8 which used the NNPDF2.3 PDF set [46].

\section{Reconstruction methods and primary selection}

Particle-flow anti- $k_{\mathrm{T}}$ jets are used. Small-area jets, denoted AK4 jets, are defined using a distance parameter of 0.4 , whereas large-area jets are defined with a distance parameter of 0.8 and are denoted as AK8 jets.

For both searches, $\mathrm{b}$ tagging is used to identify jets that contain b-flavored hadrons (b jets). The $\mathrm{b}$ tagging is applied to AK4 jets and also subjets reconstructed as part of an AK8 jet. Depending on the search, $b$ tagging involves several secondary vertexing algorithms: the online and offline CSVv2 discriminators and the DeepCSV discriminators [47]. Offline $\mathrm{b}$ tagging working points are defined with light-flavor jet mistag rates of approximately $0.1 \%$ (tight), $1 \%$ (medium), and 10\% (loose). For AK4 jets, the tight CSVv2 b tagging working point has an efficiency of $41 \%$ while the medium $\mathrm{b}$ tagging working points have CSVv2 and DeepCSV efficiencies of 63 and $68 \%$, respectively. In the case of AK8 jets, a grooming algorithm [48] looks for jet substructure and $\mathrm{b}$ tagging is applied to the resulting subjets. For AK8 jets with $p_{\mathrm{T}}$ of around $400 \mathrm{GeV}$, the medium b tagging working point has an efficiency per subjet of about $51 \%$, whereas the loose $\mathrm{b}$ tagging working point has an efficiency per subjet of about $75 \%$. The mistag rates of jets originating from c-flavored hadrons for the tight, medium, and loose $\mathrm{b}$ tagging working points are about 2, 12, and $37 \%$, respectively.

The event selection requires that the events have at least one satisfied trigger condition among a set of unprescaled high-level trigger algorithms. The set is specific to each search strategy. The trigger conditions for the low-mass search strategy rely on online jet information and, for some trigger conditions, also on b tagging information. The event selection is dominated by the trigger condition with the lowest jet multiplicity and the lowest $p_{\mathrm{T}}$ threshold. This condition requires at least six jets with $p_{\mathrm{T}}>30 \mathrm{GeV}$ with two of them passing the $\mathrm{b}$ tagging online criteria. The trigger conditions of the high-mass search strategy consist of a scalar $p_{\mathrm{T}}$ sum trigger formed from all jets with a summed $p_{\mathrm{T}}$ 
threshold of $900 \mathrm{GeV}$ and an inclusive large-area single jet trigger with a $p_{\mathrm{T}}$ threshold of $450 \mathrm{GeV}$. The high-mass search uses three other trigger conditions that include some loose jet substructure requirements and lower thresholds on either the scalar $p_{\mathrm{T}}$ sum $(700 \mathrm{GeV})$, the inclusive single-jet $p_{\mathrm{T}}(360 \mathrm{GeV})$, or on two jets, with the higher (lower) jet $p_{\mathrm{T}}$ required to exceed 300 (200) GeV.

At least one primary vertex must be found within $24 \mathrm{~cm}$ longitudinally and within $2 \mathrm{~cm}$ radially of the center of the luminous region.

\section{Low-mass search}

The low-mass search strategy uses the invariant mass reconstructed from five AK4 jets as the main discriminating variable. The event selection requires at least six jets to conform with the trigger requirements. The selection criteria are based on the properties of the signal final state, in the cases of $\mathrm{t} \rightarrow \mathrm{bW}$ and $\mathrm{H} / \mathrm{Z} \rightarrow \mathrm{b} \overline{\mathrm{b}}$. The final state is composed of two jets coming from the $\mathrm{W}$ decay and three $\mathrm{b}$ jets (two coming from the $\mathrm{H} / \mathrm{Z}$ and one from the top quark decay). The main background processes consist of QCD multijet production and top quark pair production. These backgrounds are not expected to result in a resonance in the five-jet invariant mass variable. A reduction of the QCD multijet background is achieved by imposing $\mathrm{b}$ tagging, and by requiring events to be consistent with the presence of all of the relevant states (W, H/Z, and top quark). The presence of only one top quark candidate from the selected jets is used to reduce the t $\bar{t}$ background.

\subsection{Event selection}

The following criteria define the first part of the selection:

- Small-area jet multiplicity. The event should have at least six AK4 jets with $p_{\mathrm{T}}>$ $40 \mathrm{GeV}$ within $|\eta|<4.5$.

- Leading jets. The jets with the highest $p_{\mathrm{T}}$ (leading jets) have larger $p_{\mathrm{T}}$ in the signal than in most of the backgrounds. Therefore, the leading jets must have $p_{\mathrm{T}}>170$, 130 , and $80 \mathrm{GeV}$, for the leading, second-leading, and third-leading jets, respectively.

- $b$-tagged jets. The considered $\mathrm{T}$ quark decay leads to three $\mathrm{b}$ quarks while most backgrounds have at most two b quarks. In the signal region labeled as $3 T$, at least three b-tagged jets using the tight DeepCSV working point are required for jets with $|\eta|<2$.4. Other $\mathrm{b}$ tagging working points are used to estimate the background using control samples in data.

- $T$ candidate identification. The correct identification of a $\mathrm{H} / \mathrm{Z}$ boson and a top quark in the five-jet final state relies on a $\chi^{2}$ sorting algorithm. Here the presence of all three states, namely the $\mathrm{H} / \mathrm{Z}$, the $\mathrm{W}$, and the top quark, is exploited. The algorithm loops over jet combinations and considers two b-tagged jets for the $\mathrm{H} / \mathrm{Z}$ candidate, two jets (potentially b-tagged) for the $\mathrm{W}$ candidate, and a combination of the dijet 
$\mathrm{W}$ candidate and a b-tagged jet for the top quark candidate. These combinations of jets are used to construct the variables defined in eqs. (5.1)-(5.4):

$$
\begin{aligned}
\chi_{\mathrm{H} / \mathrm{Z}}^{2} & =\left(\frac{m_{\mathrm{H} / \mathrm{Z}}^{\mathrm{meas}}-m_{\mathrm{H} / \mathrm{Z}}^{\mathrm{MC}}}{\sigma_{\mathrm{H} / \mathrm{Z}}^{\mathrm{MC}}}\right)^{2}, \\
\chi_{\mathrm{W}}^{2} & =\left(\frac{m_{\mathrm{W}}^{\text {meas }}-m_{\mathrm{W}}^{\mathrm{MC}}}{\sigma_{\mathrm{W}}^{\mathrm{MC}}}\right)^{2}, \\
\chi_{\mathrm{t}}^{2} & =\left(\frac{m_{\mathrm{t}}^{\text {meas }}-m_{\mathrm{t}}^{\mathrm{MC}}}{\sigma_{\mathrm{t}}^{\mathrm{MC}}}\right)^{2}, \\
\chi^{2} & =\chi_{\mathrm{H} / \mathrm{Z}}^{2}+\chi_{\mathrm{W}}^{2}+\chi_{\mathrm{t}}^{2},
\end{aligned}
$$

where $m^{\text {meas }}$ denotes the measured mass quantities reconstructed from the considered combination of jets, and $m^{\mathrm{MC}}$ and $\sigma^{\mathrm{MC}}$ denote the expected mass values and standard deviations from Gaussian fits to simulated signal samples. The mass values fitted for each particle are: $m_{\mathrm{H}}^{\mathrm{MC}}=121.9 \mathrm{GeV}, m_{\mathrm{Z}}^{\mathrm{MC}}=90.9 \mathrm{GeV}, m_{\mathrm{W}}^{\mathrm{MC}}=83.8 \mathrm{GeV}$ and $m_{\mathrm{t}}^{\mathrm{MC}}=173.8 \mathrm{GeV}$; these values differ only slightly from the input world-average values [49]. For the $b \bar{b}$ decays of the Higgs and $\mathrm{Z}$ bosons, the fitted standard deviations are $\sigma_{\mathrm{H}}^{\mathrm{MC}}=13.5 \mathrm{GeV}$ and $\sigma_{\mathrm{Z}}^{\mathrm{MC}}=11.4 \mathrm{GeV}$, and for the fully hadronic decays of the $\mathrm{W}$ and top quark, they are $\sigma_{\mathrm{W}}^{\mathrm{MC}}=10.0 \mathrm{GeV}$ and $\sigma_{\mathrm{t}}^{\mathrm{MC}}=16.0 \mathrm{GeV}$. One first chooses the lowest $\chi_{\mathrm{H} / \mathrm{Z}}^{2}$ b-tagged jet pair as the $\mathrm{H} / \mathrm{Z}$ candidate and then selects the other jets making up the $\mathrm{W}$ and top quark candidates by minimizing the total $\chi^{2}$. This procedure is found to improve the signal-to-background ratio by $30 \%$ compared to simply choosing the combination with the best total $\chi^{2}$. Finally, the total $\chi^{2}$ must not exceed 15 in order to ensure good quality of the $\mathrm{H} / \mathrm{Z}, \mathrm{W}$, and top quark candidates. It is found that the five jets are correctly identified about 73 and $64 \%$ of the time for the narrow width tHbq and tZbq cases, respectively.

- Second top quark mass. A large fraction of $t \bar{t}$ events survive the requirement on at least three b-tagged jets. These originate from incorrect $b$ tagging of the jet arising from a charm quark from the $\mathrm{W}$ boson part of one of the top quark decays. In order to reduce this background, we define the second top quark mass as the invariant mass formed by the $\mathrm{H} / \mathrm{Z}$ candidate and the remaining highest $p_{\mathrm{T}}$ jet not used in the $\chi^{2}$ calculation. For t't events, the second top quark mass has a peak around $172 \mathrm{GeV}$ and there are nearly no signal events expected in that region. Therefore we require that the second top quark mass is greater than $250 \mathrm{GeV}$. This leads to about a factor of two reduction in the $t \overline{\mathrm{t}}$ background.

- $b \bar{b}$ mass. Finally, the reconstructed boson from the $\mathrm{T}$ candidate must have a mass larger than $100 \mathrm{GeV}$, if looking for a $\mathrm{H}$, and the mass must be smaller than $100 \mathrm{GeV}$, if looking for a Z . This ensures that there is no overlap between the two channels.

The second part of the selection uses the presence of a top quark and a Higgs or Z boson in the event. The variables are chosen to be as model independent as possible and 
the selection criteria are optimized using the figure of merit described in [50]. The selection criteria are described below.

- Relative $H_{\mathrm{T}}$. The relative $H_{\mathrm{T}}$ variable is defined as $\left(p_{\mathrm{T}}\left(\mathrm{H} / \mathrm{Z}_{\text {cand }}\right)+p_{\mathrm{T}}\left(\mathrm{t}_{\text {cand }}\right)\right) / H_{\mathrm{T}}$. In single $\mathrm{T}$ quark production, most of the momentum should be carried by the top quark and $\mathrm{H} / \mathrm{Z}$ candidates, therefore the relative $H_{\mathrm{T}}$ is an extremely good discriminator against $\mathrm{t} \overline{\mathrm{t}}$ and multijet events. The $\mathrm{H} / \mathrm{Z}$ and $\mathrm{t}$ candidates from the $\mathrm{T}$ candidate decay must have a relative $H_{\mathrm{T}}$ greater than 0.40 .

- $\operatorname{Max}\left(\chi^{2}\right)$. The maximum among the $\chi^{2}$ values defined in eqs. (5.1)-(5.3) is examined and is required to be less than 3.0. This criterion is highly correlated to the $\chi^{2}$ criteria but represents a tighter condition that ensures that each mass is identified with high quality. It is equivalent to requiring a mass window of at most $\pm \sqrt{3} \sigma$ for each candidate.

- $\Delta R$ of jets from $H / Z$ decay. Because of the large mass of the $\mathrm{T}$ quark (above $0.6 \mathrm{TeV}$ ), the $\mathrm{H} / \mathrm{Z}$ decay tends to be boosted (but the b jets not completely merged). A small spatial separation of $\Delta R\left(\mathrm{~b}_{\mathrm{H} / \mathrm{Z}}, \mathrm{b}_{\mathrm{H} / \mathrm{Z}}\right)<1.1$ between the two b-tagged jets is required, leading to a reduction of the background. The $\Delta R$ is defined as the inter-jet separation in $\eta$ - $\phi$ space $\left(\Delta R=\sqrt{(\Delta \eta)^{2}+(\Delta \phi)^{2}}\right)$, where $\Delta \eta$ and $\Delta \phi$ are the corresponding inter-jet separations in pseudorapidity and azimuth (in radians).

- $H / Z \chi^{2}$. As most of the backgrounds do not contain a genuine Higgs boson, $\chi_{\mathrm{H} / \mathrm{Z}}^{2}$ is a very discriminating criterion for the Higgs boson decay channel. We require $\chi_{\mathrm{H}}^{2}<1.5$ for the $\mathrm{H}$ case and $\chi_{\mathrm{Z}}^{2}<1.0$ for the $\mathrm{Z}$ case. It is equivalent to a mass window of $\pm 16.5 \mathrm{GeV}$ for the Higgs boson and $\pm 11.4 \mathrm{GeV}$ for the $\mathrm{Z}$ boson. A tighter $\chi^{2}$ requirement is made for $\mathrm{Z}$ candidates to avoid background contamination from lower masses and to reduce overlap with $\mathrm{H}$ candidates.

- $\Delta R$ of jets from $W$ decay. Given the Lorentz boost of the $\mathrm{W}$ in signal events, a requirement of $\Delta R\left(j_{\mathrm{W}}, j_{\mathrm{W}}\right)<1.75$ reduces the QCD multijet background, while retaining most of the signal.

- $\Delta R$ of jets from top quark decay. The top quark decay products tend to be Lorentzboosted (but the jets do not completely merge) for the signal. A spatial separation between the b-tagged jet and the $\mathrm{W}$ candidate that is used to make the top quark candidate of $\Delta R\left(\mathrm{~b}_{\mathrm{t}}, \mathrm{W}\right)<1.2$ is required. This further reduces the QCD multijet background.

The total number of events selected from the data sample in the $3 T$ signal region is 615 (290 for the $\mathrm{tZ}$ selection and 325 for the $\mathrm{tH}$ selection). The number of expected signal events is 7.6 for a $\mathrm{T}$ quark mass of $0.7 \mathrm{TeV}, \Gamma / m_{\mathrm{T}}=0.01$, left-handed chirality, and a $\mathrm{T}$ quark produced in association with a bottom quark with a product of cross section and branching fraction of $89 \mathrm{fb}$ for each channel. For this signal process, the selection efficiency is presented in table 1 together with various simulated background processes for the Higgs and $\mathrm{Z}$ boson decay channels. 


\begin{tabular}{|lcccc|}
\hline Selection for $\mathrm{tH}$ & Signal & QCD Multijet & $\mathrm{t} \overline{\mathrm{t}}$ & Other backgrounds \\
\hline Basic selection $\left(m_{\mathrm{b} \overline{\mathrm{b}}}>100 \mathrm{GeV}\right)$ & $23.1 \pm 0.9$ & $9360 \pm 810$ & $2612 \pm 28$ & $353 \pm 23$ \\
Relative $H_{\mathrm{T}}>0.4$ & $81.4 \%$ & $42.8 \%$ & $51.9 \%$ & $52.9 \%$ \\
$\operatorname{Max}\left(\chi^{2}\right)<3.0$ & $54.3 \%$ & $14.1 \%$ & $25.1 \%$ & $21.8 \%$ \\
$\Delta R\left(\mathrm{~b}_{\mathrm{H}}, \mathrm{b}_{\mathrm{H}}\right)<1.1$ & $44.4 \%$ & $7.5 \%$ & $11.9 \%$ & $8.9 \%$ \\
$\chi_{\mathrm{H}}^{2}<1.5$ & $39.8 \%$ & $4.9 \%$ & $9.3 \%$ & $7.1 \%$ \\
$\Delta R\left(j_{\mathrm{W}}, j_{\mathrm{W}}\right)<1.75$ & $33.7 \%$ & $3.2 \%$ & $7.2 \%$ & $5.6 \%$ \\
$\Delta R\left(\mathrm{~b}_{\mathrm{t}}, \mathrm{W}\right)<1.2$ & $25.7 \%$ & $1.9 \%$ & $4.5 \%$ & $2.5 \%$ \\
Full selection & $5.9 \pm 0.4$ & $181 \pm 52$ & $116.5 \pm 6.1$ & $9.3 \pm 0.6$ \\
Selection for $\mathrm{tZ}$ & Signal & $\mathrm{QCD} \mathrm{Multijet}$ & $\mathrm{t} \overline{\mathrm{t}}$ & Other backgrounds \\
\hline Basic selection $\left(m_{\mathrm{b} \overline{\mathrm{b}}}<100 \mathrm{GeV}\right)$ & $5.7 \pm 0.2$ & $6810 \pm 630$ & $1270 \pm 17$ & $223 \pm 24$ \\
Relative $H_{\mathrm{T}}>0.4$ & $86.9 \%$ & $48.5 \%$ & $47.2 \%$ & $57.5 \%$ \\
Max $\left(\chi^{2}\right)<3.0$ & $53.3 \%$ & $15.9 \%$ & $24.1 \%$ & $28.8 \%$ \\
$\Delta R\left(\mathrm{~b}_{\mathrm{Z}}, \mathrm{b}_{\mathrm{Z}}\right)<1.1$ & $51.1 \%$ & $11.7 \%$ & $16.4 \%$ & $22.7 \%$ \\
$\chi_{\mathrm{Z}}^{2}<1.0$ & $45.0 \%$ & $7.3 \%$ & $11.5 \%$ & $18.4 \%$ \\
$\Delta R\left(j_{\mathrm{W}}, j_{\mathrm{W}}\right)<1.75$ & $37.6 \%$ & $5.2 \%$ & $9.6 \%$ & $9.9 \%$ \\
$\Delta R\left(\mathrm{~b}_{\mathrm{t}}, \mathrm{W}\right)<1.2$ & $28.8 \%$ & $1.5 \%$ & $5.7 \%$ & $5.5 \%$ \\
Full selection & $1.6 \pm 0.1$ & $103 \pm 38$ & $72.7 \pm 4.7$ & $8.1 \pm 3.9$ \\
\hline
\end{tabular}

Table 1. Cumulative efficiencies for the low-mass search after applying event selections for the signal and main backgrounds in the Higgs boson decay channel (upper half) and the Z boson decay channel (lower half). The first and last rows of each section give the expected numbers of events normalized to the integrated luminosity of $35.9 \mathrm{fb}^{-1}$. Uncertainties are statistical only. The signal values are for a mass of $0.7 \mathrm{TeV}, \Gamma / m_{\mathrm{T}}=0.01$, left-handed chirality, and a $\mathrm{T}$ quark produced in association with a bottom quark with a product of cross section and branching fraction of $89 \mathrm{fb}$ for each channel. The "Other backgrounds" column includes $\mathrm{W}+$ jets, $\mathrm{Z}+$ jets, single top quark, and $t \bar{t} H$ processes. It has been checked that the $t \bar{t} H$ process does not present a resonance in the $t H$ channel. The number of expected $t \bar{t} \mathrm{H}$ events is comparable to the expected $\mathrm{T}$ signal.

\subsection{Background estimation and validation}

None of the SM backgrounds are expected to result in a resonance in the five-jet invariant mass, therefore the spectrum of the background five-jet invariant mass distribution should have a monotonically decreasing shape. However, the second part of the selection criteria tends to shape the five-jet invariant mass distribution. In order to evaluate the shape of the five-jet invariant mass distribution for the background in data, two regions that are independent from the main $3 T$ signal region are defined using looser $\mathrm{b}$ tagging criteria. In these two regions it is important to ensure that no bias with respect to the selection criteria is present, as all backgrounds are estimated from data. The extraction of signal is done by fitting the signal and background simultaneously in all three regions.

The $\mathrm{b}$ tagging does not strongly influence the kinematic distributions of objects used to construct the five-jet invariant mass. Therefore we relax the $\mathrm{b}$ tagging criteria required for three of the five jets forming the $\mathrm{T}$ candidate. The first new region is called the $3 M$ signal region; it requires three medium b-tagged jets but excludes events with three tight b-tagged 
jets, bringing information on the background and possible signal shapes. The second new region is denoted as the $2 M 1 L$ signal region; in order to have significant numbers of events in this background-dominated region and to keep events with similar kinematics to the $3 T$ signal region, the $\mathrm{b}$ tagging criteria are relaxed to two medium and one loose $\mathrm{b}$-tagged jets but excluding three medium b-tagged jets.

Two additional samples are defined and used to validate the method; one is enriched in QCD multijet events, the other in t $\bar{t}$ events. In order to define the QCD multijet enriched control sample, the $\chi^{2}$ criterion is relaxed to 50 , the $\operatorname{Max}\left(\chi_{\mathrm{H} / \mathrm{Z}}^{2}, \chi_{\mathrm{W}}^{2}, \chi_{\mathrm{t}}^{2}\right)$ criterion is inverted, and $\chi_{\mathrm{t}}^{2}>1$ is required to reduce the fraction of $\mathrm{t} \overline{\mathrm{t}}$ events. The QCD multijet sample is subdivided into a $3 T$ region and a $2 M 1 L$ region (excluding $3 M$ ) based on the b-tagged jet configurations. For the t $\overline{\mathrm{t}}$ control sample, the $\chi^{2}$ criterion is relaxed to 50 , the $\operatorname{Max}\left(\chi_{\mathrm{H} / \mathrm{Z}}^{2}, \chi_{\mathrm{W}}^{2}, \chi_{\mathrm{t}}^{2}\right)$ and $\chi_{\mathrm{H} / \mathrm{Z}}^{2}$ criteria are inverted, the $\Delta R\left(\mathrm{~b}_{\mathrm{H} / \mathrm{Z}}, \mathrm{b}_{\mathrm{H} / \mathrm{Z}}\right)$ criterion is

relaxed to 1.5 , and $\chi_{\mathrm{t}}^{2}<1.5$ is required. The $\mathrm{t} \overline{\mathrm{t}}$ sample is subdivided into a $2 T 1 L$ (two tight and one loose b-tagged jets) region and a $2 M 1 L$ region (excluding $2 T 1 L$ ) based on the b-tagged jet configurations. For the $2 T 1 L(2 M 1 L)$ region, one of the tight (medium) b-tagged jets must be from the top quark candidate. A summary of the criteria changed to define each region is presented in table 2. The fraction of expected signal events is of the order of $3 \%$ in the QCD multijet region and $1 \%$ in the $t \bar{t} 2 T 1 L$ region (for a cross section times branching fraction of $600 \mathrm{fb}$ ).

Relaxing the $\mathrm{b}$ tagging requirement induces a change in the $\mathrm{b}$ tagging efficiency depending on the $p_{\mathrm{T}}$ and $\eta$ of the jet. As $p_{\mathrm{T}}$ and $\eta$ are two highly correlated variables, a reweighting procedure using $\eta$ and momentum is used. Weights are derived jet-by-jet for each channel and for each $b$ tagging working point using the QCD multijet control region. These weights reflect the differences in the efficiency between loose and medium and between medium and tight b-tagged jets. For each event, the product of the weights for all three b-tagged jets is applied to correct for the change in the $\mathrm{b}$ tagging efficiency going from $3 T$ to $3 M$ and from $3 M$ to $2 M 1 L$.

The validation of the method is done with the QCD multijet and $t \bar{t}$ control regions. The shape of the five-jet invariant mass distribution is compared between the QCD $3 T$ region and the QCD $2 M 1 L$ region reweighted as $3 T$; it is found to be satisfactory using the Kolmogorov-Smirnov test. Similarly, when comparing the shape of the five-jet invariant

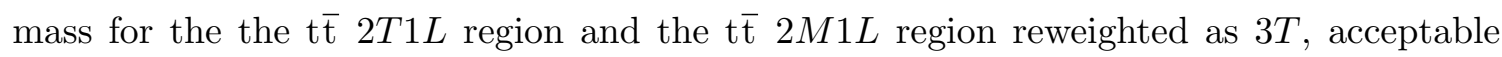
consistency is found.

The five-jet invariant mass distributions for the QCD multijet $3 T$ region, the te $2 T 1 L$ region, and the $3 M$ signal region are presented in figure 2 together with a potential signal for $m_{\mathrm{T}}=0.7 \mathrm{TeV}$ corresponding to a product of cross section times branching fraction of $600 \mathrm{fb}$. The $2 M 1 L$ region distribution is overlaid after applying the $\mathrm{b}$ tagging weight computed with respect to the $3 T, 2 T 1 L$, and $3 M$ regions, respectively. An acceptable agreement is observed in each sample.

\subsection{Low-mass search results}

For each decay channel, three independent regions based on the b-tagged jet requirements are examined: $3 T$ (largest signal over background ratio), $3 M$, and $2 M 1 L$ (background 

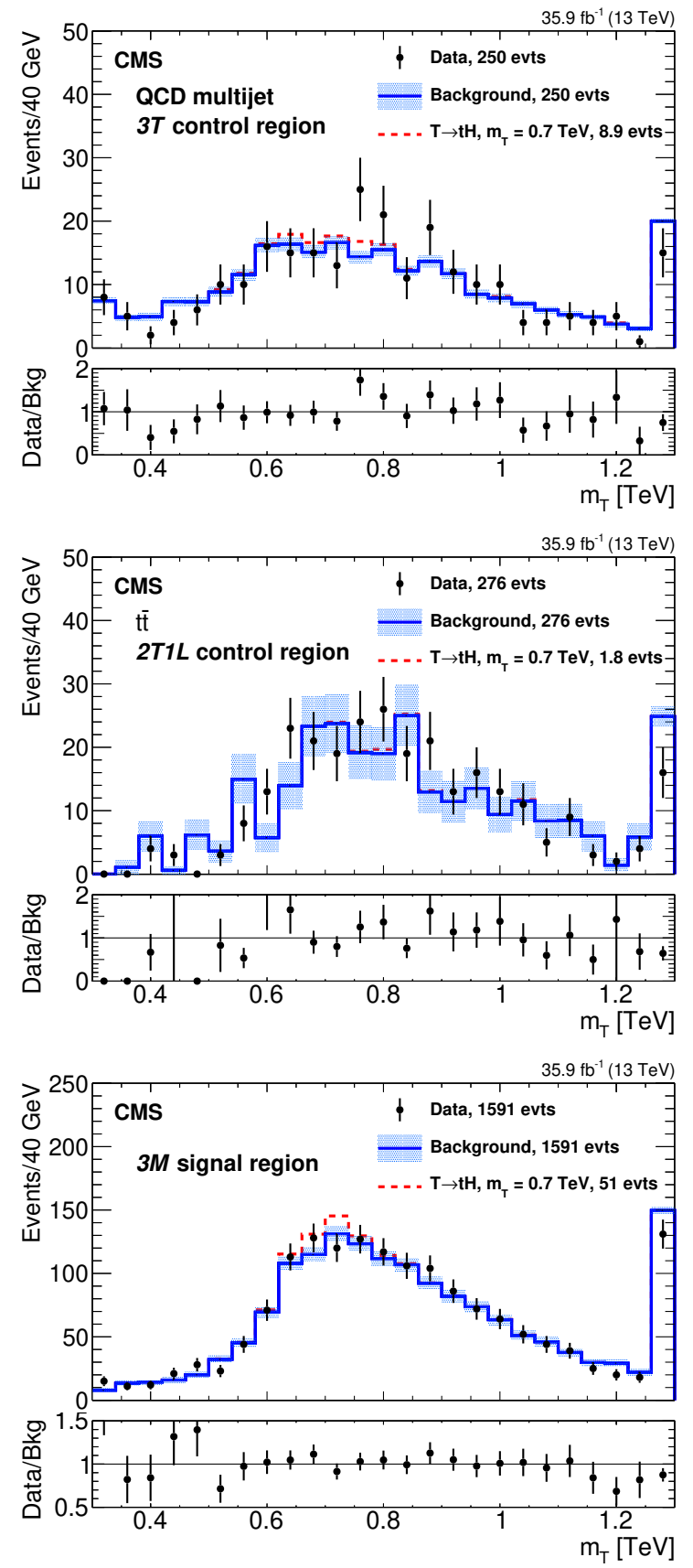

Figure 2. The five-jet invariant mass distribution (black points with error bars) for the tHbq

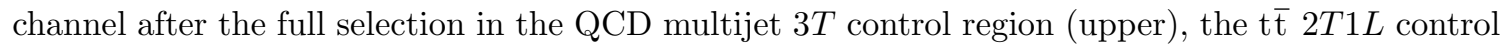
region (middle), and the $3 M$ signal region (lower). The superimposed blue histogram, labeled "background", is the reweighted $2 M 1 L$ region distribution, used as an estimate of the background shape, with its normalization adjusted to match the number of entries observed in each region. A potential narrow-width signal (dashed red histogram) is added on top of the blue histogram for $m_{\mathrm{T}}=0.7 \mathrm{TeV}$ and $\Gamma / m_{\mathrm{T}}=0.01$, for a product of signal cross section and branching fraction of $600 \mathrm{fb}$. The light blue shaded area corresponds to the statistical uncertainties in the corresponding $2 M 1 L$ region. The last bin in each distribution also contains events with masses exceeding $1.3 \mathrm{TeV}$. 


\begin{tabular}{|lll|}
\hline $3 T$ region & $3 M$ region & $2 M 1 L$ region \\
\hline $3 T$ & $*^{*} M$ but not $3 T$ & ${ }^{*} 2 M 1 L$ but not $3 M$ \\
$\chi^{2}<15$ & $\chi^{2}<15$ & $\chi^{2}<15$ \\
Relative $H_{\mathrm{T}}>0.4$ & $\operatorname{Relative~} H_{\mathrm{T}}>0.4$ & $\operatorname{Relative~} H_{\mathrm{T}}>0.4$ \\
$\operatorname{Max}\left(\chi^{2}\right)<3.0$ & $\operatorname{Max}\left(\chi^{2}\right)<3.0$ & $\operatorname{Max}\left(\chi^{2}\right)<3.0$ \\
$\Delta R\left(\mathrm{~b}_{\mathrm{H} / \mathrm{Z}}, \mathrm{b}_{\mathrm{H} / \mathrm{Z}}\right)<1.1$ & $\Delta R\left(\mathrm{~b}_{\mathrm{H} / \mathrm{Z}}, \mathrm{b}_{\mathrm{H} / \mathrm{Z}}\right)<1.1$ & $\Delta R\left(\mathrm{~b}_{\mathrm{H} / \mathrm{Z}}, \mathrm{b}_{\mathrm{H} / \mathrm{Z}}\right)<1.1$ \\
$\chi_{\mathrm{H} / \mathrm{Z}}^{2}<1.5 / 1.0$ & $\chi_{\mathrm{H} / \mathrm{Z}}^{2}<1.5 / 1.0$ & $\chi_{\mathrm{H} / \mathrm{Z}}^{2}<1.5 / 1.0$ \\
$\Delta R\left(j_{\mathrm{W}}, j_{\mathrm{W}}\right)<1.75$ & $\Delta R\left(j_{\mathrm{W}}, j_{\mathrm{W}}\right)<1.75$ & $\Delta R\left(j_{\mathrm{W}}, j_{\mathrm{W}}\right)<1.75$ \\
$\Delta R\left(\mathrm{~b}_{\mathrm{t}}, \mathrm{W}\right)<1.2$ & $\Delta R\left(\mathrm{~b}_{\mathrm{t}}, \mathrm{W}\right)<1.2$ & $\Delta R\left(\mathrm{~b}_{\mathrm{t}}, \mathrm{W}\right)<1.2$ \\
\hline
\end{tabular}

\begin{tabular}{|ll|}
\hline QCD $3 T$ region & QCD $2 M 1 L$ region \\
\hline $3 T$ & $* 2 M 1 L$ but not $3 M$ \\
$* \chi^{2}<50$ & $* \chi^{2}<50$ \\
Relative $H_{\mathrm{T}}>0.4$ & Relative $H_{\mathrm{T}}>0.4$ \\
$* 5<\operatorname{Max}\left(\chi^{2}\right)<20$ and $\chi_{\mathrm{t}}^{2}>1.0$ & $* 5<\operatorname{Max}\left(\chi^{2}\right)<20$ and $\chi_{\mathrm{t}}^{2}>1.0$ \\
$\Delta R\left(\mathrm{~b}_{\mathrm{H} / \mathrm{Z}}, \mathrm{b}_{\mathrm{H} / \mathrm{Z}}\right)<1.1$ & $\Delta R\left(\mathrm{~b}_{\mathrm{H} / \mathrm{Z}}, \mathrm{b}_{\mathrm{H} / \mathrm{Z}}\right)<1.1$ \\
$\chi_{\mathrm{H} / \mathrm{Z}}^{2}<1.5 / 1.0$ & $\chi_{\mathrm{H} / \mathrm{Z}}^{2}<1.5 / 1.0$ \\
$\Delta R\left(j_{\mathrm{W}}, j_{\mathrm{W}}\right)<1.75$ & $\Delta R\left(j_{\mathrm{W}}, j_{\mathrm{W}}\right)<1.75$ \\
$\Delta R\left(\mathrm{~b}_{\mathrm{t}}, \mathrm{W}\right)<1.2$ & $\Delta R\left(\mathrm{~b}_{\mathrm{t}}, \mathrm{W}\right)<1.2$ \\
$\mathrm{t} \overline{\mathrm{t}} 2 T 1 L$ region & $\mathrm{t} \overline{\mathrm{t}} 2 M 1 L$ region \\
\hline$* 2 T 1 L$ & $* 2 M 1 L$ but not $2 T 1 L$ \\
$*$ Top b-tag $T$ & $*$ Top b-tag $M$ \\
$* \chi^{2}<50$ & $* \chi^{2}<50$ \\
$\operatorname{Relative~} H_{\mathrm{T}}>0.4$ & Relative $H_{\mathrm{T}}>0.4$ \\
$* 3<\operatorname{Max}\left(\chi^{2}\right)<5$ & $* 3<\operatorname{Max}\left(\chi^{2}\right)<5$ \\
$* \Delta R\left(\mathrm{~b}_{\mathrm{H} / \mathrm{Z}}, \mathrm{b}_{\mathrm{H} / \mathrm{Z}}\right)<1.5$ & $* \Delta R\left(\mathrm{~b}_{\mathrm{H} / \mathrm{Z}}, \mathrm{b}_{\mathrm{H} / \mathrm{Z}}\right)<1.5$ \\
$* \chi_{\mathrm{t}}^{2}<1.5$ and $\chi_{\mathrm{H} / \mathrm{Z}}^{2}>3$ & $* \chi_{\mathrm{t}}^{2}<1.5$ and $\chi_{\mathrm{H} / \mathrm{Z}}^{2}>3$ \\
$\Delta R\left(j_{\mathrm{W}}, j_{\mathrm{W}}\right)<1.75$ & $\Delta R\left(j_{\mathrm{W}}, j_{\mathrm{W}}\right)<1.75$ \\
$\Delta R\left(\mathrm{~b}_{\mathrm{t}}, \mathrm{W}\right)<1.2$ & $\Delta R\left(\mathrm{~b}_{\mathrm{t}}, \mathrm{W}\right)<1.2$ \\
\hline
\end{tabular}

Table 2. Criteria defining the various signal and control regions. The first line of each section gives the $\mathrm{b}$ tagging requirements. The criteria that differ are preceded by an asterisk " $*$ ". The $\mathrm{b} \overline{\mathrm{b}}$ mass requirements are different for the $\mathrm{H}\left(m_{\mathrm{b} \overline{\mathrm{b}}}>100 \mathrm{GeV}\right)$ and $\mathrm{Z}$ channels $\left(m_{\mathrm{b} \overline{\mathrm{b}}}<100 \mathrm{GeV}\right)$.

dominated). The overall background shape and normalization is driven by the observations in the $2 M 1 L$ region. The background shape is linked between the regions by two transfer functions; these are derived from the $\mathrm{b}$ tagging weights to correct for $\mathrm{b}$ tagging differences between the regions. One transfer function links the $3 T$ region to the $3 M$ region and the other links the $3 M$ region to the $2 M 1 L$ region. The transfer functions, based on simple parametrizations of the dependence of the reweighting values on the five-jet invariant mass, are displayed in figure 3 . 

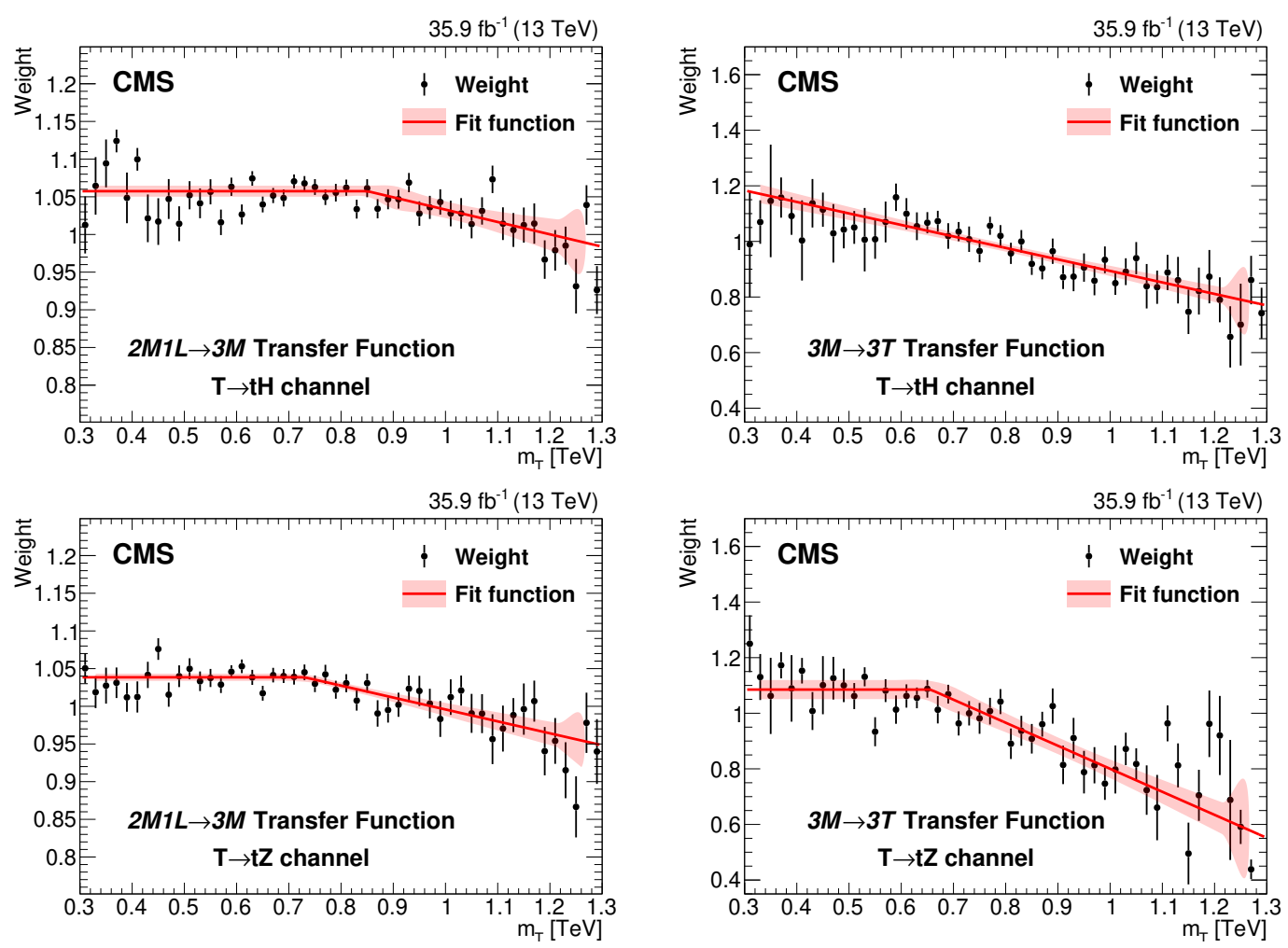

Figure 3. Dependence of the reweighting values (product of all b tagging weights) on the five-jet invariant mass for the $2 M 1 L$ region (left) and for the $3 M$ region (right), in the case of the $\mathrm{tH}$ channel (upper) and the tZ channel (lower). These variations are fitted to obtain the transfer functions (in red) using either a 3-parameter function with a constant term and a slope, or a 2parameter straight line. The light red shaded regions represent the central $68 \%$ CL interval for each fit when taking into account only the statistical uncertainties.

The signal is parametrized as a Gaussian shape following the fit of the T quark reconstructed mass for each of the simulation samples for each region. The variations of the Gaussian fit parameters (mean and standard deviation) with $\mathrm{T}$ quark mass are fitted for each region. The parametrizations for the $\mathrm{tH}$ and $\mathrm{tZ}$ channels are found to be compatible. The systematic uncertainties are discussed in section 7 . Here we note simply that they are all taken as correlated between the channels, except the ones related to the transfer functions and the normalization between regions. For a given channel, the fit procedure adjusts the shape of the background bin by bin based on the data in each of the regions, taking into account the transfer function between regions. The overall fit uses $40 \mathrm{GeV}$ wide bins; it includes three bin-independent fit parameters, namely the signal strength and two relative normalization factors between each region, and fit parameters for the background contribution in each bin of the $2 M 1 L$ region.

The background-only post-fit invariant mass distributions for each of the regions $(2 M 1 L, 3 M$, and $3 T)$ as well as for each channel ( $\mathrm{tZ}$ and $\mathrm{tH})$ are displayed in figure 4. A signal with a mass of $m_{\mathrm{T}}=0.7 \mathrm{TeV}$ and product of the cross section and branching fraction of $600 \mathrm{fb}$ is superimposed. An excess is observed when fitting the three regions for 

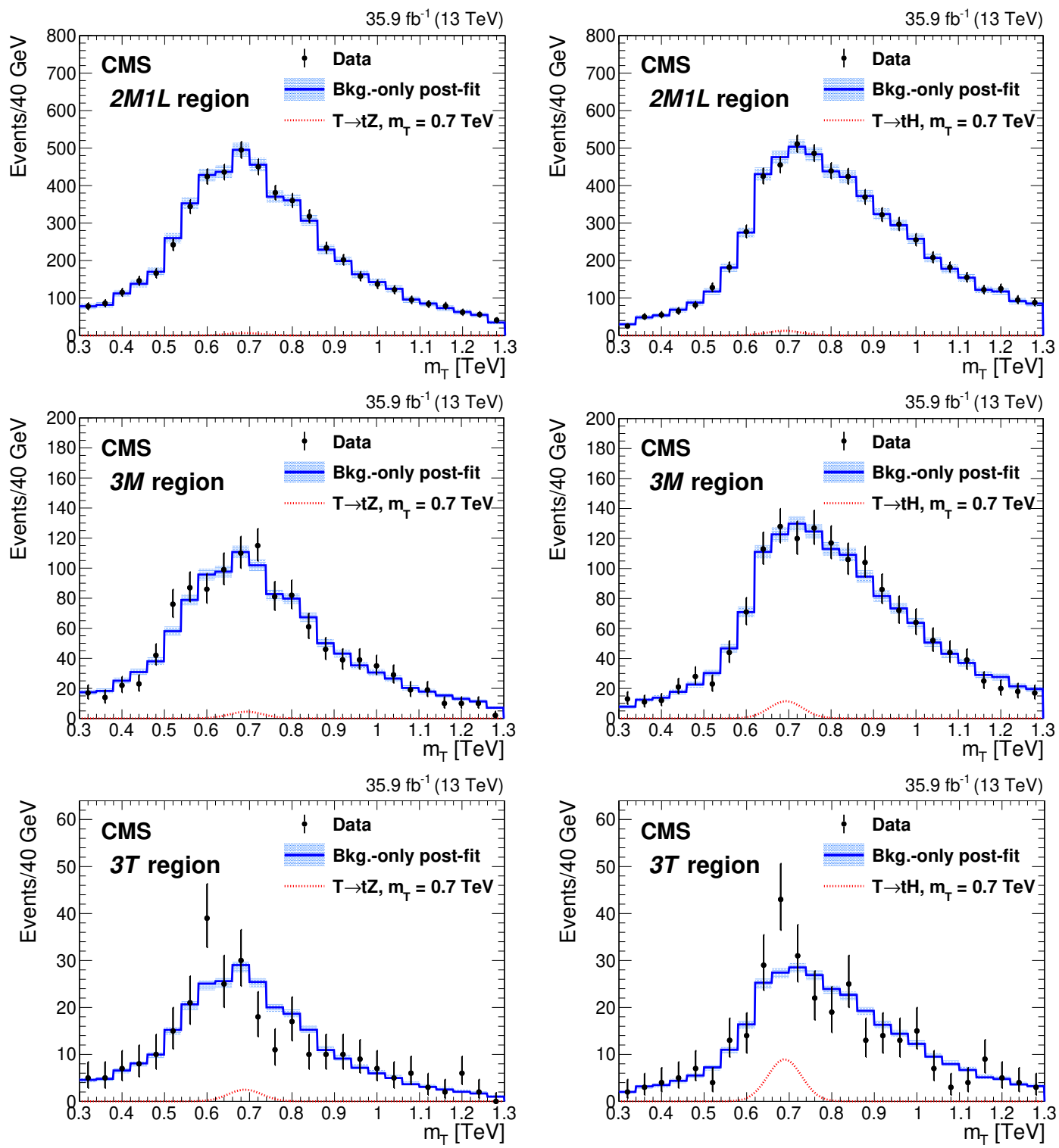

Figure 4. The background-only post-fit invariant mass distributions for the $\mathrm{tZ}$ candidates (left) and $\mathrm{tH}$ candidates (right) for each region fitted: $2 M 1 L$ (upper row), $3 M$ (middle row), and $3 T$ (lower row). The signal hypothesis shown is a $\mathrm{T}$ with a mass of $0.7 \mathrm{TeV}$, narrow width, and a product of the cross section and branching fraction of $600 \mathrm{fb}$ for the tZbq and tHbq channels. The data are represented by the black points with error bars, the signal hypothesis is represented by the red dashed line, the blue histogram gives the fitted background, and the light blue band represents the uncertainty in the background fit.

the tH channel. The local significance is 3.0 standard deviations for a $\mathrm{T}$ quark mass of $0.68 \mathrm{TeV}$. For the same T quark mass the local significance is 0.2 standard deviations in the $\mathrm{tZ}$ channel. In a search for a vector-like quark, one expects similar branching fractions for the $\mathrm{tH}$ and $\mathrm{tZ}$ channels. No overall excess is measured when considering the fit of all six distributions. 
The result of the median value for the limit in terms of the appropriate cross section is calculated using the asymptotic $\mathrm{CL}_{\mathrm{s}}$ framework [51-53].

Similarly, results are obtained in the cases where the T quark has 10, 20, and $30 \%$ widths and in the case of production in association with a top quark. In all cases, the observed results show no evidence for a signal. The studies have also been performed for the case of right-handed chirality. No effect of the chirality is observed, indicating that the low-mass search is insensitive to this property. The resulting limits are reported in section 8 .

\section{High-mass search}

This search strategy focuses on reconstructing the invariant mass of $\mathrm{T} \rightarrow \mathrm{tH}$ and $\mathrm{T} \rightarrow \mathrm{tZ}$ candidates formed from two large-area jets in the fully hadronic channel. The large-area jets are associated with events in which the top quark and the Higgs or Z boson are each highly Lorentz-boosted, and correspondingly the search targets $\mathrm{T}$ masses of $1 \mathrm{TeV}$ and above. The background consists mostly of top quark pair production and QCD multijet production.

\subsection{Particle tagging}

To identify boosted $\mathrm{t} \rightarrow \mathrm{bW} \rightarrow \mathrm{bq} \overline{\mathrm{q}}^{\prime}, \mathrm{H} \rightarrow \mathrm{b} \overline{\mathrm{b}}$, and $\mathrm{Z} \rightarrow \mathrm{b} \overline{\mathrm{b}}$ decays, jet substructure techniques [54] are used, which remove soft and collinear radiation from the clustered jet constituents. Clusters of the remaining constituents are identified with each of the quarks from the decay of the original particle. The soft-drop algorithm [48, 55] is used to groom the jets, using the soft radiation fraction parameter $z=0.1$ and the angular exponent parameter $\beta=0$. The algorithm yields two soft-drop subjets. The jet mass after applying the soft-drop algorithm will be referred to as the soft-drop mass. The pruning grooming algorithm [56] is also used, leading to the pruned jet mass. For pruning, the minimum subjet $p_{\mathrm{T}}$ as a fraction of the parent jet $p_{\mathrm{T}}$ is required to exceed 0.1 and the separation angle in $\eta$ - $\phi$ space between the two subjets must exceed 0.5. Furthermore, the $N$-subjettiness algorithm [57] is used to further select jets with three or two substructures for the top quark jets, and the $\mathrm{H}$ and $\mathrm{Z}$ boson jets, respectively. Flavor tagging is applied to identify b quark subjets using the CSVv2 multivariate discriminator, in order to further enhance the signal purity and suppress backgrounds from non-t $\bar{t}+$ jets multijet processes. The particle tagging criteria for boosted top quark, and $\mathrm{H}$ and $\mathrm{Z}$ boson jets are as follows:

- $H$ jet: an AK8 jet with $p_{\mathrm{T}}>300 \mathrm{GeV}$ must have a pruned jet mass within the range $105-135 \mathrm{GeV}$. The ratio of the $N$-subjettiness variables $\tau_{2} / \tau_{1}$ of the jet is required to be $<0.6$. At least one of the two soft-drop subjets must pass the medium $b$ tag criterion and the other subjet should pass at least the loose $\mathrm{b}$ tag criterion.

- $Z$ jet: an $\mathrm{AK} 8$ jet with $p_{\mathrm{T}}>200 \mathrm{GeV}$ must have a pruned jet mass within the range $65-105 \mathrm{GeV}$. The requirements on the $N$-subjettiness ratio $\tau_{2} / \tau_{1}$ and subjet $\mathrm{b}$ tagging are the same as those for the $\mathrm{H}$ jet. 
- $t$ jet: an AK8 jet with $p_{\mathrm{T}}>400 \mathrm{GeV}$ and a soft-drop mass within the range 105$220 \mathrm{GeV}$ is required. The jet should have an $N$-subjettiness ratio $\tau_{3} / \tau_{2}<0.57$, indicating that the large-area jet is likely to have three subjets. The soft-drop subjet with the highest CSVv2 discriminator value should pass the medium b tag criterion [58].

In addition, reversed-H-tagged, reversed-Z-tagged, and reversed-t-tagged jets are defined, with the same kinematic and $N$-subjettiness requirements as those for their tagged counterparts, but with complementary b tagging criteria, as follows:

- Reversed-H-tagged jet: same criteria as for an H-tagged jet but with one subjet passing the medium $b$ tag criterion and the other subjet failing the loose $b$ tag criterion.

- Reversed-Z-tagged jet: same criteria as for a Z-tagged jet but with both subjets failing the loose $b$ tag criterion.

- Reversed-t-tagged jet: same criteria as for a t-tagged jet but with the highest softdrop subjet $\mathrm{b}$ discriminant failing the medium $\mathrm{b}$ tag criterion.

The reversed- $Z$ tag is defined differently from the reversed- $H$ tag so that sensitivity to a potential $\mathrm{tZ}$ signal, including efficiency from $\mathrm{Z} \rightarrow \mathrm{q} \overline{\mathrm{q}}(\mathrm{q} \neq \mathrm{b})$ decays, can be retained.

\subsection{Event selection}

Only events satisfying the following primary selection criteria are considered further, either as candidates for the signal or for the associated background control regions:

- Small-area jet multiplicity: at least four AK4 jets with $p_{\mathrm{T}}>30 \mathrm{GeV}$ and $|\eta|<5$.

- Large-area jet multiplicity: at least two AK8 jets with $p_{\mathrm{T}}>200 \mathrm{GeV}$ and $|\eta|<2.4$.

- Leading jet: the highest- $p_{\mathrm{T}} \mathrm{AK} 8$ jet should have $p_{\mathrm{T}}>400 \mathrm{GeV}$ and a pruned mass greater than $50 \mathrm{GeV}$.

- $S c a l a r p_{\mathrm{T}}$ sum: the scalar sum of the transverse momenta of the two highest- $p_{\mathrm{T}}$ AK8 jets should exceed $850 \mathrm{GeV}$.

- Extra jet multiplicity: at least two of the AK4 jets should be separated by a distance $\Delta R>1.2$ from the two leading AK8 jets.

- Forward extra jet: at least one of the extra AK4 jets defined above should have $|\eta|>2.4$.

The last two criteria are imposed in order to ensure evidence that the selected events contain a diquark (bq or tq) system that is produced in association with the $\mathrm{T}$ quark, where the quark (q) associated with the vector boson tends to be forward. Events passing the selection requirements above are by design expected to be almost fully efficient for the trigger requirements. 
Signal candidates passing the primary event selection are further categorized according to the following criteria. At this stage, the focus is on choosing the best pair of AK8 jets for constructing the $\mathrm{T}$ candidate mass.

- Double-tag: each of the two highest- $p_{\mathrm{T}}$ selected AK8 jets must have either a t tag or an $\mathrm{H} / \mathrm{Z}$ tag. Furthermore, one of these jets must have a $t$ tag and the other an $\mathrm{H} / \mathrm{Z}$ tag. In the ambiguous case, where both jets are t-tagged and $\mathrm{H} / \mathrm{Z}$-tagged, the higher- $p_{\mathrm{T}}$ jet is assigned as the top quark candidate, and the lower- $p_{\mathrm{T}}$ jet as the Higgs/Z boson candidate.

- $H / Z$ tag isolation: motivated by reducing $\mathrm{t} \overline{\mathrm{t}}$ background, events are rejected if any AK4 jet is separated from the $\mathrm{H} / \mathrm{Z}$ candidate jet by $0.55<\Delta R(j, \mathrm{H} / \mathrm{Z})<0.9$.

Signal regions, $\mathrm{S}_{\mathrm{H}}$ and $\mathrm{S}_{\mathrm{Z}}$, are defined using these criteria for the $\mathrm{tH}$ and $\mathrm{tZ}$ searches respectively. Related background control regions are defined and are described in section 6.3.

We denote the measured AK8 jet four-vectors corresponding to the top quark, Higgs boson and $\mathrm{Z}$ candidates as $P_{\mathrm{t}}=\left(E_{\mathrm{t}}, \vec{p}_{\mathrm{t}}\right), P_{\mathrm{H}}=\left(E_{\mathrm{H}}, \vec{p}_{\mathrm{H}}\right)$ and $P_{\mathrm{Z}}=\left(E_{\mathrm{Z}}, \vec{p}_{\mathrm{Z}}\right)$ and construct a corrected $\mathrm{T}$ mass-sensitive observable, $\widetilde{m}_{\mathrm{T}}$. This observable takes advantage of the knowledge of the top quark and Higgs/Z boson masses to correct the masses of the AK8 jets; it is inspired by a similar variable used in ref. [59] that was based on a suggestion in ref. [60]. The reconstructed mass of the $\mathrm{T}$ candidate from the $\mathrm{tX}$ dijet system $\left(m_{\mathrm{tX}}^{j}\right)$, with $\mathrm{X}=\mathrm{H} / \mathrm{Z}$, is adjusted for deviations of the reconstructed top quark and Higgs/Z boson AK8 masses $\left(m_{\mathrm{t}}^{j}, m_{\mathrm{X}}^{j}\right)$ from the known $\mathrm{t}$ and $\mathrm{H} / \mathrm{Z}$ masses [49], as follows:

$$
\widetilde{m}_{\mathrm{T}}=\sqrt{\left(P_{\mathrm{t}}+P_{\mathrm{X}}\right)^{2}}-\sqrt{P_{\mathrm{t}}^{2}}-\sqrt{P_{\mathrm{X}}^{2}}+m_{\mathrm{t}}+m_{\mathrm{X}}=m_{\mathrm{tX}}^{j}-\left(m_{\mathrm{t}}^{j}-m_{\mathrm{t}}\right)-\left(m_{\mathrm{X}}^{j}-m_{\mathrm{X}}\right),
$$

where the $j$ superscripts denote jet-based measured mass quantities. This estimator is found to have better performance in terms of mass resolution by about $10 \%$ compared to the uncorrected mass estimator. It has also been verified that it is accompanied by a commensurate reduction in background acceptance.

Example distributions of $\widetilde{m}_{\mathrm{T}}$ in the signal regions, $\mathrm{S}_{\mathrm{H}}$ and $\mathrm{S}_{\mathrm{Z}}$, are shown in figure 5 for $\mathrm{T}$ masses of 1.2 and $1.8 \mathrm{TeV}$ for both narrow and large widths. It can be seen that in the $\mathrm{S}_{\mathrm{Z}}$ region, in addition to the expected efficiency for the tZbq process, there is also substantial efficiency for the tHbq process; the reverse is not true for the $\mathrm{S}_{\mathrm{H}}$ region.

\subsection{Background estimation}

The $\widetilde{m}_{\mathrm{T}}$ distribution is used to determine the amount of signal potentially present in the data. A fit is performed that takes advantage of the relatively narrow signal shape in $\widetilde{m}_{\mathrm{T}}$ compared to the broader shape expected from the backgrounds. After the primary event selection criteria, the main backgrounds are $t \overline{\mathrm{t}}$ and QCD multijet events. The $t \overline{\mathrm{t}}$ background is estimated using simulated events and the QCD multijet background is estimated using control regions in data. Other smaller background sources, designated as "other" and

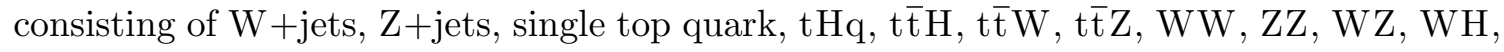



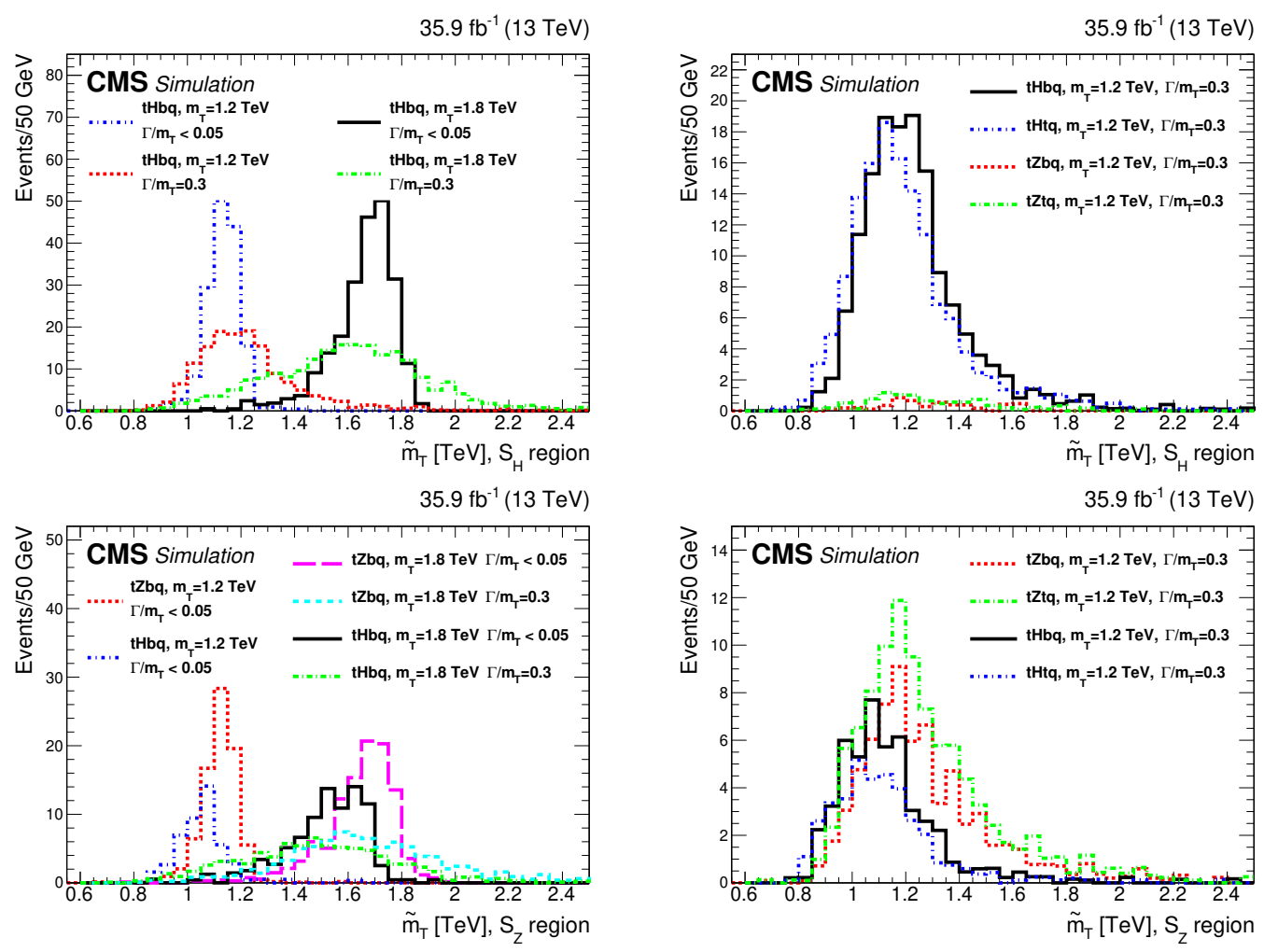

Figure 5. Example $\widetilde{m}_{\mathrm{T}}$ distributions in the signal regions, $\mathrm{S}_{\mathrm{H}}$ (upper row), and $\mathrm{S}_{\mathrm{Z}}$ (lower row). For presentation purposes, the cross sections for tHbq, tZbq, tHtq and tZtq are set equal to $1 \mathrm{pb}$ for all masses and fractional widths and normalized to the integrated luminosity of the data set. The left column illustrates potential signals with a range of masses and widths for the tHbq and tZbq channels. The right column illustrates potential signals for one mass and a large width for all four processes including also tHtq and tZtq.

and $\mathrm{ZH}$, are all estimated from simulation. Background templates are constructed using a smoothing procedure that fits the $\widetilde{m}_{\mathrm{T}}$ distributions with an empirical functional form. A simultaneous fit is then performed in eight regions: four regions designed to test for $\mathrm{tH}$ signal contributions and four regions designed to test for $\mathrm{tZ}$ signal contributions. The fit examines all eight regions and fits the $\widetilde{m}_{\mathrm{T}}$ distributions for the amounts of signal and QCD multijet background contributions using t $\bar{t}$ and other backgrounds predicted from simulation. Fits are performed under three hypothetical signal scenarios, tH only, tZ only, and $\mathrm{tH}+\mathrm{tZ}$. In the latter case, the small difference in cross section for tHbq relative to tZbq is taken from the singlet model calculation. For tHtq relative to tZtq, the difference from the (TB) doublet model calculation is used.

The criteria described in section 6.2 define the main signal regions $\left(\mathrm{S}_{\mathrm{H}}\right.$ and $\left.\mathrm{S}_{\mathrm{Z}}\right)$ using the t-, $\mathrm{H}-$, and Z-tagged jets. The additional six mutually exclusive regions are used as control regions in the fit and to predict the shapes and normalization of the QCD multijet background from data; these are denoted $\mathrm{Q}_{\mathrm{H}}, \mathrm{T}_{\mathrm{H}}, \mathrm{R}_{\mathrm{H}}$ for the tH signal and $\mathrm{Q}_{\mathrm{Z}}, \mathrm{L}_{\mathrm{Z}}, \mathrm{R}_{\mathrm{Z}}$ for the $\mathrm{tZ}$ signal. Regions $\mathrm{Q}_{\mathrm{H}}$ and $\mathrm{Q}_{\mathrm{Z}}$ are control regions for the QCD multijet background. Region $T_{H}$ is a $t \bar{t}$ enriched control region, while region $L_{Z}$ has sensitivity to non-b $\bar{b} Z$ 


\begin{tabular}{|lcccc|}
\hline Region & Channel & First jet & Second jet & $\mathrm{H} / \mathrm{Z}$ tag isolation \\
\hline $\mathrm{Q}_{\mathrm{H}}$ & $\mathrm{tH}$ & reversed-t-tagged & reversed-H-tagged & - \\
$\mathrm{T}_{\mathrm{H}}$ & $\mathrm{tH}$ & $\mathrm{t}$ tag & reversed-H-tagged & - \\
$\mathrm{R}_{\mathrm{H}}$ & $\mathrm{tH}$ & reversed-t-tagged & $\mathrm{H}$ tag & required \\
$\mathrm{S}_{\mathrm{H}}$ & $\mathrm{tH}$ & $\mathrm{t}$ tag & $\mathrm{H}$ tag & required \\
$\mathrm{Q}_{\mathrm{Z}}$ & $\mathrm{tZ}$ & reversed-t-tagged & reversed-Z-tagged & - \\
$\mathrm{L}_{\mathrm{Z}}$ & $\mathrm{tZ}$ & $\mathrm{t}$ tag & reversed-Z-tagged & - \\
$\mathrm{R}_{\mathrm{Z}}$ & $\mathrm{tZ}$ & reversed-t-tagged & $\mathrm{Z}$ tag & required \\
$\mathrm{S}_{\mathrm{Z}}$ & $\mathrm{tZ}$ & $\mathrm{t}$ tag & $\mathrm{Z} \mathrm{tag}$ & required \\
\hline
\end{tabular}

Table 3. Overview of the criteria used to define the mutually exclusive $Q_{H}, T_{H}, R_{H}, S_{H}, Q_{Z}$, $\mathrm{L}_{\mathrm{Z}}, \mathrm{R}_{\mathrm{Z}}$, and $\mathrm{S}_{\mathrm{Z}}$ regions. These are based on the particle tagging criteria for $\mathrm{t}, \mathrm{H}$, and $\mathrm{Z}$ jets and for the reversed-t-tagged, reversed-H-tagged, and reversed-Z-tagged jets using the two highest $p_{\mathrm{T}}$ AK8 jets.

decays. Regions $\mathrm{R}_{\mathrm{H}}$ and $\mathrm{R}_{\mathrm{Z}}$ serve as control regions that test the rejection of QCD multijet events by the $\mathrm{H}$ tag and $\mathrm{Z}$ tag criteria.

For the definition of the regions, only the two highest $p_{\mathrm{T}} \mathrm{AK} 8$ jets are examined and each jet must be either tagged or reversed-tagged. The signal region $\mathrm{S}_{\mathrm{H}}$ requires a t-tagged jet and an $\mathrm{H}$-tagged jet. Region $\mathrm{Q}_{\mathrm{H}}$ requires a reversed-t-tagged jet and a reversed-H-tagged jet. Region $\mathrm{T}_{\mathrm{H}}$ requires a t-tagged jet and a reversed-H-tagged jet. Region $R_{H}$ requires a reversed-t-tagged jet and an $H$-tagged jet. Regions $R_{H}$ and $S_{H}$ include the isolation requirement on the $\mathrm{H}$-tagged jet, while regions $\mathrm{Q}_{\mathrm{H}}$ and $\mathrm{T}_{\mathrm{H}}$ do not include an isolation requirement around the reversed-H-tagged jet. These choices define a $T_{H}$ region that is enhanced in $t \bar{t}$ events, thus providing a suitable control region. The order of assigning events starts with region $S_{H}$, then proceeds with region $T_{H}, R_{H}$, and then $\mathrm{Q}_{\mathrm{H}}$, where each subsequent region is not allowed to contain any of the events assigned to the previous region. In ambiguous cases, as is done for the signal region, the higher- $p_{\mathrm{T}}$ jet is assigned as the t-tagged or reversed-t-tagged jet.

There are four analogous regions designated by $\mathrm{Q}_{\mathrm{Z}}, \mathrm{L}_{\mathrm{Z}}, \mathrm{R}_{\mathrm{Z}}$, and $\mathrm{S}_{\mathrm{Z}}$ for events with $\mathrm{t}-$ and Z-tagged jets for events that do not satisfy the $\mathrm{Q}_{\mathrm{H}}, \mathrm{T}_{\mathrm{H}}, \mathrm{R}_{\mathrm{H}}$, or $\mathrm{S}_{\mathrm{H}}$ region definitions. The $\mathrm{Q}_{Z}$ region has a reversed-t-tagged jet and a reversed-Z-tagged jet. The $\mathrm{L}_{\mathrm{Z}}$ region has a t-tagged jet and a reversed-Z-tagged jet. The $R_{Z}$ region has a reversed-t-tagged jet and a $\mathrm{Z}$-tagged jet. These criteria make region $\mathrm{L}_{\mathrm{Z}}$ sensitive to hadronic decays of $\mathrm{Z}$ bosons other than $\mathrm{b} \overline{\mathrm{b}}$ from both the $\mathrm{T} \rightarrow \mathrm{tZ}$ signal and the background. Regions $R_{Z}$ and $\mathrm{S}_{Z}$ include the isolation requirement on the $\mathrm{Z}$-tagged jet, while regions $\mathrm{Q}_{\mathrm{Z}}$ and $\mathrm{L}_{\mathrm{Z}}$ do not include an isolation requirement around the reversed-Z-tagged jet. Events can only be assigned to one of the eight regions. These criteria lead to a well-defined $\widetilde{m}_{\mathrm{T}}$ value for each event corresponding to the mass assignments implicit in the tagging criteria. Table 3 summarizes the criteria for the eight regions.

A simultaneous fit is performed to the $\widetilde{m}_{\mathrm{T}}$ distributions in each of the eight regions to determine the amount of signal present. The signal templates are taken directly from the 
simulated signal samples. The t $\bar{t}$ and other background contributions are found using the smoothed templates. The smoothed QCD multijet background shape is determined from the data in region $Q_{H}$ for the regions $Q_{H}, T_{H}, R_{H}$, and $S_{H}$ and in region $Q_{Z}$ for the $Q_{Z}$, $\mathrm{L}_{\mathrm{Z}}, \mathrm{R}_{\mathrm{Z}}$, and $\mathrm{S}_{\mathrm{Z}}$ regions. A binned likelihood fit is performed using the $\widetilde{m}_{\mathrm{T}}$ variable, with 50 bins of $50 \mathrm{GeV}$ width over the range $0.6-3.1 \mathrm{TeV}$. All background components, except QCD multijet, are constrained within uncertainties using predictions from MC simulations. The numbers of QCD multijet events in regions $\mathrm{S}_{\mathrm{H}}$ and $\mathrm{S}_{\mathrm{Z}}$ are estimated using the control regions. The amount of QCD multijet background in each of the control regions $\mathrm{Q}_{\mathrm{H}}$, $T_{H}, R_{H}, Q_{Z}, L_{Z}$, and $R_{Z}$ is found from the following. If the expected numbers of QCD multijet events in the four regions are $N_{\mathrm{Q}_{\mathrm{H}}}, N_{\mathrm{T}_{\mathrm{H}}}, N_{\mathrm{R}_{\mathrm{H}}}, N_{\mathrm{S}_{\mathrm{H}}}$ then, if the Higgs boson and reversed-Higgs boson tagging are independent of the top quark tagging and reversed-top quark tagging criteria, one may write:

$$
\frac{N_{\mathrm{S}_{\mathrm{H}}}}{N_{\mathrm{T}_{\mathrm{H}}}}=\frac{N_{\mathrm{R}_{\mathrm{H}}}}{N_{\mathrm{Q}_{\mathrm{H}}}} \Rightarrow N_{\mathrm{S}_{\mathrm{H}}}=N_{\mathrm{T}_{\mathrm{H}}} \frac{N_{\mathrm{R}_{\mathrm{H}}}}{N_{\mathrm{Q}_{\mathrm{H}}}} .
$$

Using eq. (6.2), one may then make a data-based prediction of the number of QCD multijet events in the signal region $\mathrm{S}_{\mathrm{H}}$. Similarly, the number of QCD multijet background events in region $\mathrm{S}_{\mathrm{Z}}$ can be estimated from $N_{\mathrm{S}_{\mathrm{Z}}}=N_{\mathrm{L}_{\mathrm{Z}}}\left(N_{\mathrm{R}_{\mathrm{Z}}} / N_{\mathrm{Q}_{\mathrm{Z}}}\right)$. In our fitting method, $N_{\mathrm{Q}_{\mathrm{H}}}$, $N_{\mathrm{T}_{\mathrm{H}}}, N_{\mathrm{R}_{\mathrm{H}}}, N_{\mathrm{Q}_{\mathrm{Z}}}, N_{\mathrm{L}_{\mathrm{Z}}}$, and $N_{\mathrm{R}_{\mathrm{Z}}}$ are free parameters determined by the fit. The fit assumes that the double ratios $\left(N_{\mathrm{Q}_{\mathrm{H}}} / N_{\mathrm{R}_{\mathrm{H}}}\right) /\left(N_{\mathrm{T}_{\mathrm{H}}} / N_{\mathrm{S}_{\mathrm{H}}}\right)$ and $\left(N_{\mathrm{Q}_{\mathrm{Z}}} / N_{\mathrm{R}_{\mathrm{Z}}}\right) /\left(N_{\mathrm{L}_{\mathrm{Z}}} / N_{\mathrm{S}_{\mathrm{Z}}}\right)$ are consistent with unity in order to constrain the number of QCD multijet events in regions $\mathrm{S}_{\mathrm{H}}$ and $\mathrm{S}_{\mathrm{Z}}$.

The double ratios measured from the QCD multijet simulation are

$$
\left(N_{\mathrm{Q}_{\mathrm{H}}} / N_{\mathrm{R}_{\mathrm{H}}}\right) /\left(N_{\mathrm{T}_{\mathrm{H}}} / N_{\mathrm{S}_{\mathrm{H}}}\right)=0.77 \pm 0.39 \pm 0.21
$$

and

$$
\left(N_{\mathrm{Q}_{\mathrm{Z}}} / N_{\mathrm{R}_{\mathrm{Z}}}\right) /\left(N_{\mathrm{L}_{\mathrm{Z}}} / N_{\mathrm{S}_{\mathrm{Z}}}\right)=0.67 \pm 0.35 \pm 0.24
$$

which are both consistent with the expected value of unity within their combined statistical and systematic uncertainties. The systematic uncertainties are discussed in section 7 . The predictions of the QCD multijet contributions, which include the overall effect of the shape and the normalization, have been validated using a fit that uses a different set of eight control regions in data. These control regions are mutually exclusive to the previously defined eight regions, contain larger numbers of events, and are defined using loose b tagging criteria on the $t$ tag and the reversed-t tag in a sample that excludes events with forward $(|\eta|>2.4)$ jets. The double ratio is taken to be fully correlated between the $t \mathrm{H}$ and $\mathrm{tZ}$ regions, with a central value of 1.0 , and an assigned uncertainty of 0.6 . This uncertainty is assessed based on the measured double ratios from the relatively low number of events in the QCD multijet simulation. The fits to data are observed to be insensitive to the exact uncertainty used in that the preferred value for the double ratio tends to be close to unity.

Limits on the signal strength are extracted by fitting the signal and backgrounds to the data. The fit finds the amount of signal as well as the amounts of QCD multijet background in each of the eight regions. The QCD multijet event yields in the regions $\mathrm{Q}_{\mathrm{H}}$, $T_{H}$, and $R_{H}$ are allowed to float freely. The QCD multijet event yield in the region $S_{H}$ 


\begin{tabular}{|lcccccc|}
\hline Data set & $m_{\mathrm{T}}$ & $\sigma$ & $\mathrm{Q}_{\mathrm{H}}$ & $\mathrm{T}_{\mathrm{H}}$ & $\mathrm{R}_{\mathrm{H}}$ & $\mathrm{S}_{\mathrm{H}}$ \\
\hline $\mathrm{t} \overline{\mathrm{t}}+$ jets & - & - & $140 \pm 20$ & $230 \pm 30$ & $16.8 \pm 3.8$ & $21.7 \pm 4.8$ \\
Other background & - & - & $21.7 \pm 9.6$ & $20.5 \pm 7.0$ & $7.4 \pm 4.4$ & $3.0 \pm 1.6$ \\
QCD multijet & - & - & $478 \pm 42$ & $91 \pm 35$ & $125 \pm 12$ & $28.4 \pm 9.1$ \\
Total background & - & - & $640 \pm 28$ & $342 \pm 23$ & $149 \pm 12$ & $53.1 \pm 7.7$ \\
Data & - & - & 640 & 345 & 151 & 52 \\
tHbq & 1.2 & 142 & $6.80 \pm 0.30$ & $10.7 \pm 0.4$ & $11.6 \pm 0.4$ & $20.6 \pm 0.6(0.40)$ \\
$\mathrm{tZbq}$ & 1.2 & 131 & $1.56 \pm 0.15$ & $1.18 \pm 0.13$ & $0.49 \pm 0.08$ & $0.73 \pm 0.11(0.02)$ \\
$\mathrm{tHtq}$ & 1.2 & 40.7 & $2.32 \pm 0.10$ & $3.70 \pm 0.13$ & $3.21 \pm 0.12$ & $5.63 \pm 0.16(0.39)$ \\
$\mathrm{tZtq}$ & 1.2 & 32.9 & $0.47 \pm 0.03$ & $0.49 \pm 0.03$ & $0.13 \pm 0.01$ & $0.14 \pm 0.02(0.01)$ \\
$\mathrm{tHbq}$ & 1.8 & 13.6 & $1.08 \pm 0.04$ & $1.54 \pm 0.05$ & $1.83 \pm 0.05$ & $2.83 \pm 0.07(0.58)$ \\
$\mathrm{tZbq}$ & 1.8 & 11.0 & $0.12 \pm 0.01$ & $0.13 \pm 0.01$ & $0.08 \pm 0.01$ & $0.07 \pm 0.01(0.02)$ \\
$\mathrm{tHtq}$ & 1.8 & 4.0 & $0.33 \pm 0.01$ & $0.49 \pm 0.01$ & $0.50 \pm 0.01$ & $0.76 \pm 0.02(0.53)$ \\
$\mathrm{tZtq}$ & 1.8 & 3.2 & $0.11 \pm 0.01$ & $0.11 \pm 0.01$ & $0.03 \pm 0.01$ & $0.04 \pm 0.01(0.03)$ \\
\hline
\end{tabular}

Table 4. Post-fit numbers of events for the $\mathrm{Q}_{\mathrm{H}}, \mathrm{T}_{\mathrm{H}}, \mathrm{R}_{\mathrm{H}}$, and $\mathrm{S}_{\mathrm{H}}$ regions for the data and specified background sources, for the overall eight-region background-only fit. The uncertainties include both the statistical and systematic components. The fitted background sums depend on the data. The expected event yields for various signal samples are also listed with statistical uncertainties only, along with the corresponding masses $(\mathrm{TeV})$ and cross sections $(\mathrm{fb})$. The fractional width considered is $30 \%$. The percent efficiency in region $\mathrm{S}_{\mathrm{H}}$ is also noted in parentheses, alongside the event yield.

is constrained using eq. (6.2) with the double ratio being modeled with a Gaussian prior. The same procedure is used for regions $\mathrm{Q}_{\mathrm{Z}}, \mathrm{L}_{\mathrm{Z}}, \mathrm{R}_{\mathrm{Z}}$, and $\mathrm{S}_{\mathrm{Z}}$. All other uncertainties are treated either using log-normal priors (for those that change the event yields only), or as Gaussian priors (with shape variations corresponding to the \pm 1 standard deviation change in those uncertainties that affect the $\widetilde{m}_{\mathrm{T}}$ distributions as well as the yields). The fitting method is validated with a data sample based on simulation. The fit uses the modified frequentist approach for confidence levels, taking the profile likelihood ratio as the test statistic [51, 52] and using the asymptotic approximation for limit setting [53].

\subsection{High-mass search results}

Table 4 gives the total number of events in regions $\mathrm{Q}_{\mathrm{H}}, \mathrm{T}_{\mathrm{H}}, \mathrm{R}_{\mathrm{H}}$, and $\mathrm{S}_{\mathrm{H}}$, while table 5 gives the total number of events in regions $\mathrm{Q}_{\mathrm{Z}}, \mathrm{L}_{\mathrm{Z}}, \mathrm{R}_{\mathrm{Z}}$, and $\mathrm{S}_{\mathrm{Z}}$. These tables also show the fitted contributions from each background source for the background-only hypothesis fit when fitting the observed $\widetilde{m}_{\mathrm{T}}$ distributions in the eight regions. Also included in the tables are the expected numbers of events and efficiencies for various signals. The efficiencies are inclusive; they include all decay modes of the $\mathrm{H}, \mathrm{Z}$, and $\mathrm{t}$ quark.

The resulting post-fit $\widetilde{m}_{\mathrm{T}}$ distributions in data based on the background-only hypothesis are shown for the $\mathrm{Q}_{\mathrm{H}}, \mathrm{T}_{\mathrm{H}}, \mathrm{R}_{\mathrm{H}}, \mathrm{S}_{\mathrm{H}}$ and $\mathrm{Q}_{\mathrm{Z}}, \mathrm{L}_{\mathrm{Z}}, \mathrm{R}_{\mathrm{Z}}, \mathrm{S}_{\mathrm{Z}}$ regions in figures 6 and 7 , respectively. It is found that these post-fit distributions are consistent with the backgroundonly model with an acceptable goodness-of-fit. Upper limits are then set on the cross 


\begin{tabular}{|lcccccc|}
\hline Data set & $m_{\mathrm{T}}$ & $\sigma$ & $\mathrm{Q}_{\mathrm{Z}}$ & $\mathrm{L}_{\mathrm{Z}}$ & $\mathrm{R}_{\mathrm{Z}}$ & $\mathrm{S}_{\mathrm{Z}}$ \\
\hline $\mathrm{t} \overline{\mathrm{t}}+$ jets & - & - & $258 \pm 32$ & $421 \pm 53$ & $16.4 \pm 4.4$ & $30.2 \pm 5.8$ \\
Other background & - & - & $271 \pm 64$ & $223 \pm 94$ & $12.1 \pm 4.0$ & $2.4 \pm 1.5$ \\
QCD multijet & - & - & $5710 \pm 150$ & $830 \pm 230$ & $259 \pm 19$ & $45.0 \pm 9.7$ \\
Total background & - & - & $6230 \pm 120$ & $1480 \pm 180$ & $288 \pm 17$ & $77.5 \pm 9.7$ \\
Data & - & - & 6253 & 1475 & 286 & 80 \\
tHbq & 1.2 & 142 & $6.44 \pm 0.30$ & $10.1 \pm 0.4$ & $3.46 \pm 0.22$ & $6.97 \pm 0.33(0.14)$ \\
tZbq & 1.2 & 131 & $27.3 \pm 0.6$ & $45.6 \pm 0.8$ & $6.01 \pm 0.29$ & $9.87 \pm 0.39(0.21)$ \\
tHtq & 1.2 & 40.7 & $2.22 \pm 0.09$ & $3.42 \pm 0.12$ & $0.93 \pm 0.06$ & $1.55 \pm 0.08(0.11)$ \\
tZtq & 1.2 & 32.9 & $4.10 \pm 0.08$ & $6.71 \pm 0.10$ & $0.83 \pm 0.04$ & $1.41 \pm 0.05(0.12)$ \\
tHbq & 1.8 & 13.6 & $1.12 \pm 0.04$ & $1.48 \pm 0.05$ & $0.66 \pm 0.03$ & $1.09 \pm 0.04(0.22)$ \\
tZbq & 1.8 & 11.0 & $3.98 \pm 0.07$ & $5.64 \pm 0.09$ & $0.89 \pm 0.04$ & $1.21 \pm 0.04(0.31)$ \\
tHtq & 1.8 & 4.0 & $0.28 \pm 0.01$ & $0.44 \pm 0.01$ & $0.15 \pm 0.01$ & $0.23 \pm 0.01(0.16)$ \\
tZtq & 1.8 & 3.2 & $1.27 \pm 0.02$ & $1.83 \pm 0.03$ & $0.24 \pm 0.01$ & $0.37 \pm 0.01(0.32)$ \\
\hline
\end{tabular}

Table 5. Post-fit numbers of events for the $\mathrm{Q}_{\mathrm{Z}}, \mathrm{L}_{\mathrm{Z}}, \mathrm{R}_{\mathrm{Z}}$, and $\mathrm{S}_{\mathrm{Z}}$ regions for the data and specified background sources, for the overall eight-region background-only fit. The uncertainties include both the statistical and systematic components. The fitted background sums depend on the data. The expected event yields for various signal samples are also listed with statistical uncertainties only, along with the corresponding masses $(\mathrm{TeV})$ and cross sections $(\mathrm{fb})$. The fractional width considered is $30 \%$. The percent efficiency in region $\mathrm{S}_{\mathrm{Z}}$ is also noted in parentheses, alongside the event yield.

sections for the two production modes ( $\mathrm{pp} \rightarrow \mathrm{Tbq}$ and $\mathrm{pp} \rightarrow \mathrm{Ttq}$ ). These upper limits are reported in section 8 together with the limits from the low-mass search for four fractional width $\left(\Gamma / m_{\mathrm{T}}\right)$ values and the two decay modes $(\mathrm{tH}$ and $\mathrm{tZ})$ as well as their sum $(\mathrm{tH}+\mathrm{tZ})$.

\section{Systematic uncertainties}

The systematic uncertainties can be classified into those that affect the overall yields of the signal and the background processes, and those that affect the invariant mass distributions $m_{\mathrm{T}}$ (for the low-mass search) and $\widetilde{m}_{\mathrm{T}}$ (for the high-mass search). The sources of systematic uncertainties and their effects on the signal and the background are summarized in table 6 .

The trigger efficiency for the low-mass category is measured in data and is found to be about $97 \%$ with an assigned uncertainty of $3 \%$. The trigger efficiency for the high-mass analysis is measured using hadronic triggers to be over $99.5 \%$. There is a mild dependence on $\widetilde{m}_{\mathrm{T}}$ that is evaluated using a muon-based monitor trigger. The maximum variation is $3 \%$ and this is taken as the uncertainty in the overall event yields.

The jet energy scale uncertainties depend on the $p_{\mathrm{T}}$ and $\eta$ of the jets [61]. The jet energy resolution in data is found to be worse than that in simulation, and the discrepancy is corrected by applying an extra smearing to the energy of jets in simulated events. Both the jet energy scale and resolution uncertainties affect the overall scale and shapes of the invariant mass distributions. 

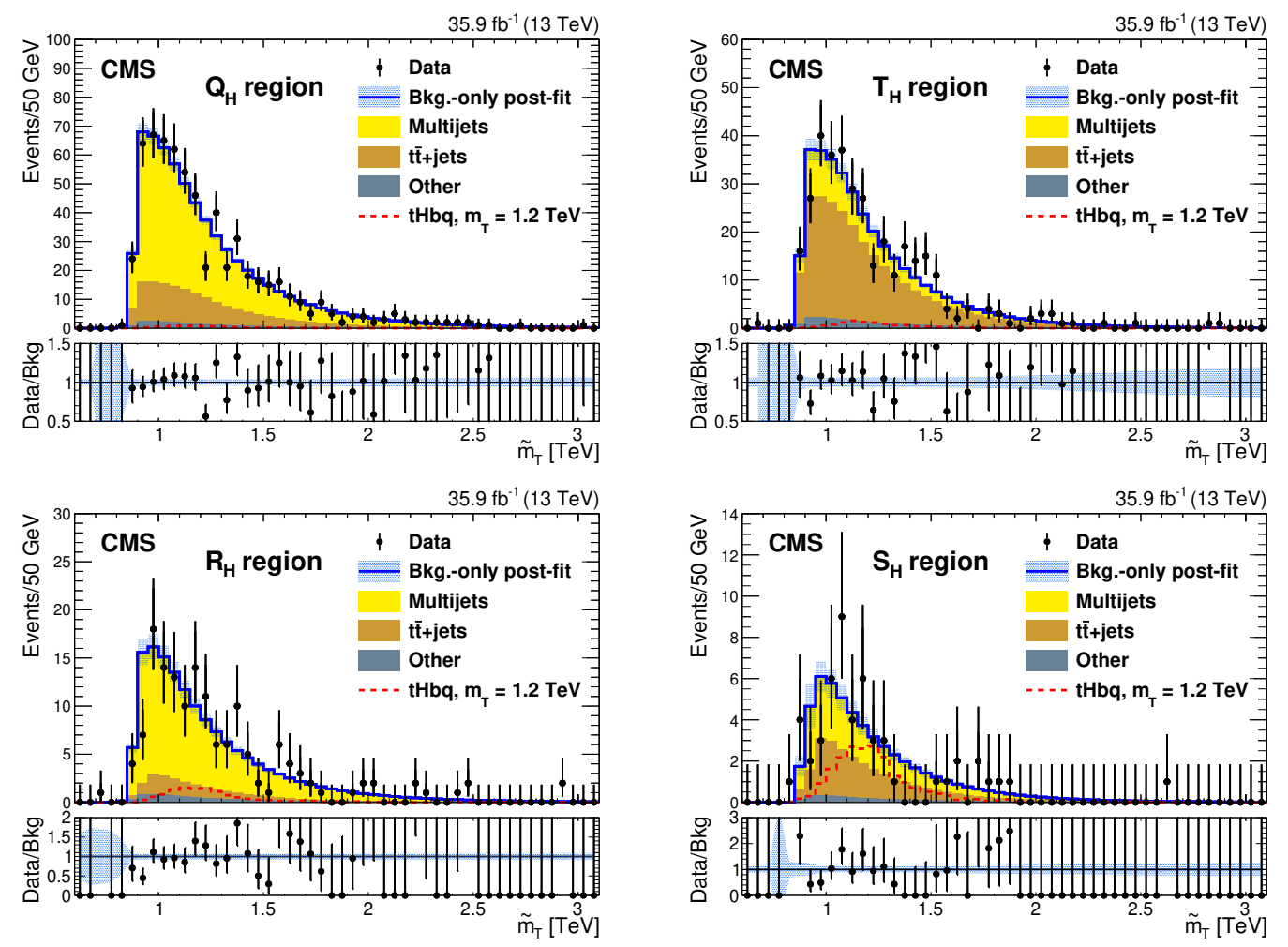

Figure 6. The background-only post-fit distributions in data for the $\mathrm{Q}_{\mathrm{H}}, \mathrm{T}_{\mathrm{H}}, \mathrm{R}_{\mathrm{H}}$, and $\mathrm{S}_{\mathrm{H}}$ regions that are used as signal and control regions primarily for the $\mathrm{T} \rightarrow \mathrm{tH}$ channel. The upper plots show regions $\mathrm{Q}_{\mathrm{H}}$ (left) and $\mathrm{T}_{\mathrm{H}}$ (right), while the lower plots show regions $\mathrm{R}_{\mathrm{H}}$ (left) and $\mathrm{S}_{\mathrm{H}}$ (right). The dashed red histogram is an example $\mathrm{T} \rightarrow \mathrm{tH}$ signal for the tHbq process with a $1.2 \mathrm{TeVT}$ quark mass and a fractional width of $30 \%$ with a cross section from the singlet model of $142 \mathrm{fb}$. The lower panels show the ratio of observed data to fitted background per bin. The error bars on the data represent $68 \%$ CL Poisson intervals. The light blue band in each ratio panel shows the fractional uncertainties in the fitted background.

The uncertainties in the $\mathrm{H}$ and $\mathrm{Z}$ jet mass scale and resolution and the $N$-subjettiness selection [61] affect the high-mass search. The jet mass scale, resolution, and the $\mathrm{N}$ subjettiness selection efficiency were measured in a sample of $t \bar{t}+$ jets events with one top quark decaying leptonically and the other top quark decaying hadronically (semileptonic $t \bar{t}+$ jets events). The hadronically decaying top quark is boosted enough to produce a merged $\mathrm{W} \rightarrow \mathrm{q} \overline{\mathrm{q}}^{\prime}$ jet separated from a b jet. The jet mass scale was evaluated to be unity with an uncertainty of $2 \%$. An additional uncertainty of about $10 \%$ was derived using simulations to account for the difference between the jet showering for a $\mathrm{W} \rightarrow \mathrm{q} \overline{\mathrm{q}}^{\prime}$ jet and an $\mathrm{H} \rightarrow \mathrm{b} \overline{\mathrm{b}}$ or $\mathrm{Z} \rightarrow \mathrm{b} \overline{\mathrm{b}}$ jet, using parton shower models from PYTHIA 8 and HERWIG ++2.7 .1 [62]. The EE5C tune [63] is used for HERWIG ++ . The jet mass resolution was found to be $23 \%$ larger in the data than in the simulation, with an uncertainty of $18 \%$. The ratio of the $N$-subjettiness selection efficiency in the data to the simulation (scale factor) was found to be $1.11 \pm 0.08$ with an additional jet $p_{\mathrm{T}}$-dependent uncertainty. The jet mass scale and $N$-subjettiness scale factor uncertainties affect only the event yields whereas the jet mass resolution affects both the yields and the $\widetilde{m}_{\mathrm{T}}$ distributions. 

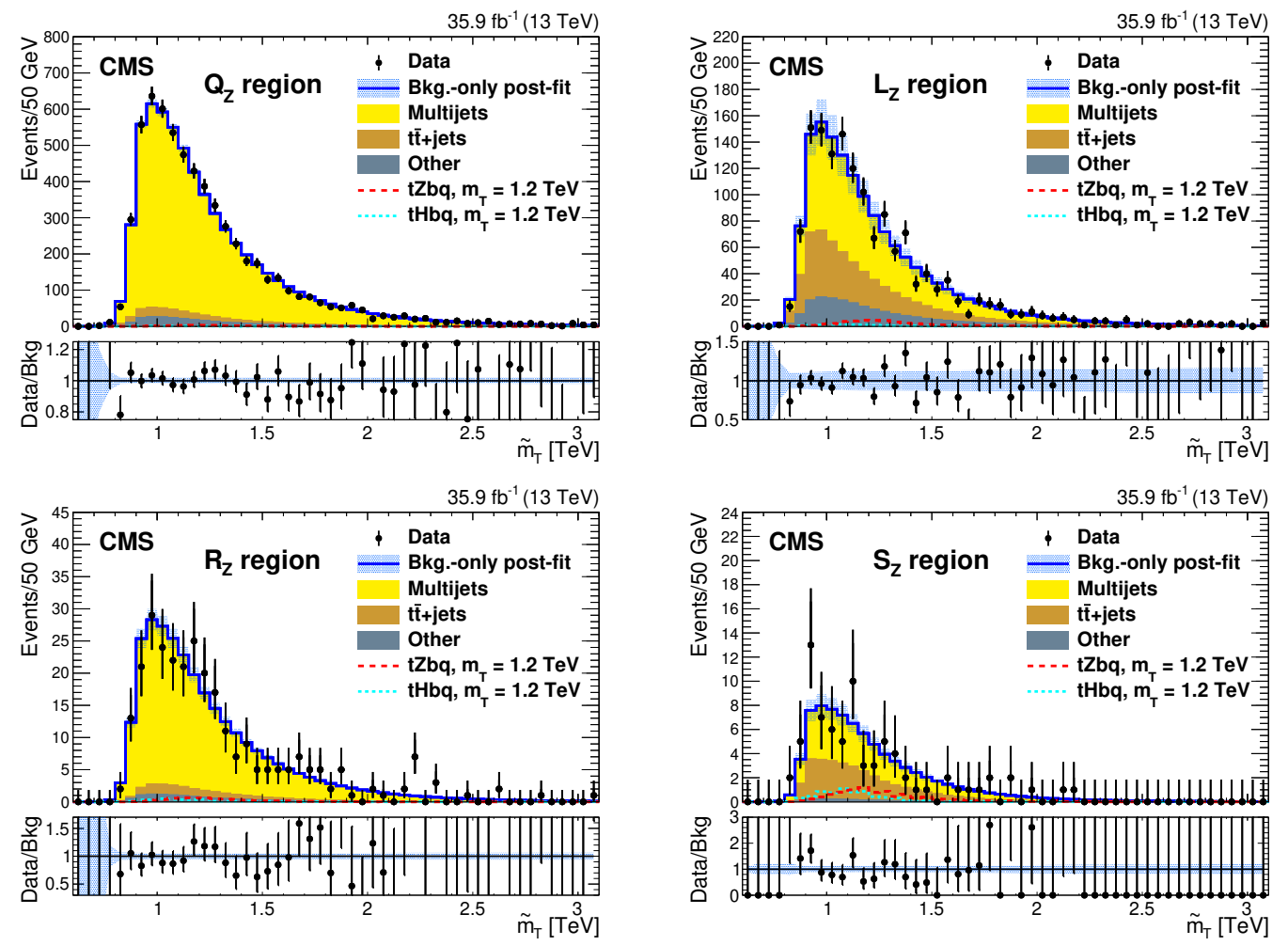

Figure 7. The background-only post-fit distributions in data for the $\mathrm{Q}_{Z}, \mathrm{~L}_{\mathrm{Z}}, \mathrm{R}_{\mathrm{Z}}$, and $\mathrm{S}_{\mathrm{Z}}$ regions that are used as signal and control regions primarily for the $\mathrm{T} \rightarrow \mathrm{tZ}$ channel. The upper plots show regions $\mathrm{Q}_{\mathrm{Z}}$ (left) and $\mathrm{L}_{\mathrm{Z}}$ (right), while the lower plots show regions $\mathrm{R}_{\mathrm{Z}}$ (left) and $\mathrm{S}_{\mathrm{Z}}$ (right). The dashed red histogram is an example $\mathrm{T} \rightarrow \mathrm{tZ}$ signal for the $\mathrm{tZbq}$ process with a 1.2 TeVT quark mass and a fractional width of $30 \%$ with a cross section from the singlet model of $131 \mathrm{fb}$. The shorter dashed cyan histogram is for $\mathrm{T} \rightarrow \mathrm{tH}$ signal for the tHbq process with the model assumptions used in figure 6 . The lower panels show the ratio of observed data to fitted background per bin. The error bars on the data represent $68 \%$ CL Poisson intervals. The light blue band in each ratio panel shows the fractional uncertainties in the fitted background.

The $\mathrm{b}$ tagging [47] efficiency scale factor uncertainties for AK4 jets are measured in multijets and $t \bar{t}+$ jets samples, separately for $\mathrm{b}$ quark jets and for light-quark and gluon jets. These uncertainties affect only the signal event yields for the low-mass analysis. For the high-mass analysis, the $b$ tagging efficiency scale factor uncertainty for subjets is measured using multijet and $t \overline{\mathrm{t}}+$ jets samples and is applied to the subjets for $\mathrm{H}$ and $\mathrm{Z}$ jets. These affect both the event yields and the $\widetilde{m}_{\mathrm{T}}$ distributions.

For the top quark jet tagging used in the high-mass search, the scale factor is measured using semileptonic $t \bar{t}+$ jets events, with a boosted top quark jet, using the method from ref. [58]. The scale factor with uncertainty is $1.07_{-0.03}^{+0.05}$ for each top quark jet, and affects both the event yields and the $\widetilde{m}_{\mathrm{T}}$ distributions.

The PDF uncertainties were evaluated with the PDF4LHC procedure [64], by reweighting the simulated events using the eigenvectors of the NNPDF3.0 PDF set; it was found to change the overall event yields by $0.5-1 \%$. The renormalization and factorization scale 


\begin{tabular}{|c|c|c|}
\hline Source & Low-mass & High-mass \\
\hline \multicolumn{3}{|c|}{ Signal yield (\%) } \\
\hline Trigger efficiency & 3 & 3 \\
\hline Jet energy scale and resolution & Shape & Shape \\
\hline Jet mass scale and resolution & - & 2 \\
\hline $\mathrm{H} / \mathrm{Z}$ tagging correction factor & - & 10 \\
\hline $\mathrm{H} / \mathrm{Z}$ jet $\tau_{21}$ selection & - & $7+$ jet $p_{\mathrm{T}}$-dependence \\
\hline Jet mass resolution & - & Shape \\
\hline Top quark jet tagging & - & $\begin{array}{l}+5 \\
-3\end{array}$ \\
\hline b tagging selection & $5-7$ & Shape \\
\hline PDF & $0.5-1$ & $0.5-1$ \\
\hline Pileup modeling & $2-4$ & 2 \\
\hline Integrated luminosity & 2.5 & 2.5 \\
\hline \multirow[t]{2}{*}{ Renorm./fact. scales } & $1-2$ & Shape \\
\hline & \multicolumn{2}{|c|}{ Background yield (\%) } \\
\hline Low-mass background & Shape & - \\
\hline$t \bar{t}+$ jets cross section & - & ${ }_{-5.5}^{+6.1}$ \\
\hline $\mathrm{W}+$ jets cross section & - & 3.8 \\
\hline QCD multijet background & - & $23-25$ \\
\hline Renorm./fact. scales & - & Shape \\
\hline
\end{tabular}

Table 6. The systematic uncertainties in the signal and background yields for each search. The uncertainties marked "Shape" affect both the event yields and the distributions.

uncertainties were estimated by doubling and halving the nominal values used in the simulations. These were found to affect the high-mass search event yields for both the signal and the $\mathrm{t} \overline{\mathrm{t}}+$ jets background.

The uncertainty in the measurement of the integrated luminosity amounts to $2.5 \%$ [65] and affects the overall event yields of all simulated processes. The uncertainty associated with the mismodeling of pileup is evaluated based on a $4.6 \%$ variation on the pp total inelastic cross section [66] and affects the overall simulated event yields for both analyses.

The systematic uncertainties in the background estimation for the low-mass search are taken into account by inflating the uncertainty in the slope parameter of the transfer functions. This includes an uncertainty component justified from the difference observed when the weights are computed on light-flavor versus heavy-flavor jets and an uncertainty component coming from the uncertainties in the fit arising from the $\mathrm{b}$ tagging efficiency. Based on the observed differences, the slope parameter uncertainty is increased by a factor of four for the $2 M 1 L$ to $3 M$ region transfer function and by a factor of three for the $3 M$ to $3 T$ region transfer function. For the high-mass search, the uncertainty in the background prediction includes the systematic uncertainty in the double ratio used to constrain the QCD multijet event yields in the different control and the signal regions (section 6.3). While the initial uncertainty in this double ratio is assigned to be $60 \%$, the postfit uncertainty ranges from $23-25 \%$, depending on signal model. 
The $t \overline{\mathrm{t}}+$ jets cross section uncertainty is ${ }_{-5.5}^{+6.1 \%}$ at next-to-next-to-leading order [67], while that in the $\mathrm{W}+$ jets cross section is $3.8 \%[68,69]$. These uncertainties affect only the high-mass search where both these backgrounds are taken from simulations. These uncertainties are propagated to the estimation of the QCD multijet background from the data, where the non-QCD multijet background components are subtracted from the data. The uncertainties also include $\widetilde{m}_{\mathrm{T}}$-dependent modeling uncertainties for the various background components.

\section{Search results}

The low-mass search is sensitive to masses from 0.6 to $1.2 \mathrm{TeV}$, while the high-mass search is sensitive to masses from 0.7 to $2.6 \mathrm{TeV}$, but with its main sensitivity starting around $1 \mathrm{TeV}$. The limits presented here correspond to a confidence level of $95 \%$. They are based on whichever of the two searches for each considered mass has the best estimated expected sensitivity for each decay channel ( $\mathrm{tH}, \mathrm{tZ}$, and the sum), production mode, and fractional width. The limits from each search considered separately are presented for completeness in appendix A.

Upper limits are set on the cross sections for the two production modes ( $\mathrm{pp} \rightarrow \mathrm{Tbq}$ and $\mathrm{pp} \rightarrow \mathrm{Ttq}$ ) with the two decay modes ( $\mathrm{tH}$ and $\mathrm{tZ})$ as well as their sum $(\mathrm{tH}+\mathrm{tZ})$, for four fractional width $\left(\Gamma / m_{\mathrm{T}}\right)$ values. The individual limits for the tH decay mode neglect potential contributions from the $\mathrm{tZ}$ and $\mathrm{bW}$ decay modes and, similarly, the individual limits for the $\mathrm{tZ}$ decay mode neglect potential contributions from the $\mathrm{tH}$ and $\mathrm{bW}$ decay modes. The $(\mathrm{tH}+\mathrm{tZ})$ sum limits are computed assuming the relative cross sections for the two channels calculated in the respective model for a particular width based on ref. [70]. For $\mathrm{pp} \rightarrow \mathrm{Tbq}$, this corresponds to the $\mathrm{T}$ singlet model with $\kappa_{\mathrm{W}}=\kappa_{\mathrm{H}}=\kappa_{\mathrm{Z}}$ and for $\mathrm{pp} \rightarrow \mathrm{Ttq}$, to the (TB) doublet model with $\kappa_{\mathrm{H}}=\kappa_{\mathrm{Z}}$ and $\kappa_{\mathrm{W}}=0$, where $\kappa_{\mathrm{W}}, \kappa_{\mathrm{H}}$, and $\kappa_{\mathrm{Z}}$ denote the $\mathrm{T}$ coupling parameter to $\mathrm{bW}, \mathrm{tH}$, and $\mathrm{tZ}$, respectively. Only potential contributions from $\mathrm{bW}$ are neglected for the $(\mathrm{tH}+\mathrm{tZ})$ experimental limits. The calculated cross sections for $\mathrm{tH}$ and $\mathrm{tZ}$ are approximately the same but unequal. This is due both to mass effects explicit in the decay width expressions of ref. [5] and to additional amplitude contributions associated with the $2 \rightarrow 4$ finite width calculation arising from $\mathrm{T}$ quarkmediated Feynman diagrams relevant to the large-width regime, such as the t-channel exchange of a $\mathrm{T}$ quark. The fractional width values include 10, 20, and $30 \%$, and the narrow-width case. Given the estimated effective Gaussian mass resolution of about $5 \%$, the experimental limits set using the narrow-width simulated signal samples are applicable to (Breit-Wigner) fractional widths of up to about 5\%. The experimental upper limits on cross sections are generally more restrictive for smaller widths given the narrower line shapes. The computed cross sections are found to depend approximately linearly on the fractional width.

Figure 8 shows the cross section upper limits for Tbq production for the tHbq and tZbq channels, and their sum. The figure includes results for a narrow fractional width, corresponding to $\Gamma / m_{\mathrm{T}} \leq 0.05$, and for a fractional width of $10 \%$. Results for the same quantities are shown in figure 9 for fractional widths of 20 and $30 \%$. Similar results for 
Ttq production for the tHtq and tZtq channels, and their sum are shown in figure 10 for narrow fractional width and for $10 \%$ fractional width, and the corresponding results for fractional widths of 20 and $30 \%$ are shown in figure 11. Superimposed on these results are the expected cross sections for the T singlet model and for the (TB) doublet model. The $\mathrm{tH}$ and $\mathrm{tZ}$ branching fractions for a narrow width are both approximately $25 \%$ for the $\mathrm{T}$ singlet model and $50 \%$ for the (TB) doublet model.

The presented results are evaluated using left-handed chirality for the $\mathrm{T}$ in the $\mathrm{pp} \rightarrow$ Tbq cases and right-handed chirality for the pp $\rightarrow$ Ttq cases. Studies with the opposite chirality for narrow width $\mathrm{T} \rightarrow \mathrm{tH}$ and $\mathrm{T} \rightarrow \mathrm{tZ}$ have shown similar sensitivity with the differences being small for the low-mass search and at most $10 \%$ for the high-mass search.

The results of this search show that the observed limits are consistent with the expected limits arising from the background-only hypothesis. Depending on the fractional width, it can be seen that this search has expected sensitivity for $\mathrm{T}$ masses within the $\mathrm{T}$ singlet model up to $1.28 \mathrm{TeV}$ (for tHbq + tZbq with $30 \%$ fractional width). For $\mathrm{T}$ masses below $1 \mathrm{TeV}$, the models of the associated production with a bottom quark are strongly constrained by the observed limits from the low-mass search signature, which are generally more stringent than expected above $0.75 \mathrm{TeV}$; for the $\mathrm{T}$ singlet model masses in the range 0.70 to $1.00 \mathrm{TeV}$ are excluded at 95\% CL for some fractional widths between 5 and $30 \%$. For the T quark masses above $1.00 \mathrm{TeV}$, the observed limits are above model predictions, and so no exclusion at $95 \%$ CL is possible with this data set in this mass range. The models with the associated production with a top quark have lower cross sections with a median expected sensitivity for $\mathrm{T}$ quark masses within the (TB) doublet model of $0.82 \mathrm{TeV}$ for the largest fractional width of 30\%. However, for this model, no range of masses is excluded at 95\% CL for any of the masses and fractional widths considered here.

The presented experimental upper limits on the cross sections are of general interest in more model independent approaches and demonstrate the great potential of electroweak single production searches to test vector-like quark production at mass scales far beyond those accessible with pair production.

\section{Summary}

A search for a vector-like top quark partner $\mathrm{T}$ in the electroweak single production mode with fully hadronic final states has been performed using pp collision events at $\sqrt{s}=13 \mathrm{TeV}$ collected by the CMS experiment in 2016. The data sample corresponds to an integrated luminosity of $35.9 \mathrm{fb}^{-1}$. The $\mathrm{T}$ quarks are assumed to couple only to the standard model third-generation quarks. The decay channels exploited are $\mathrm{T} \rightarrow \mathrm{tH}$ and $\mathrm{T} \rightarrow \mathrm{tZ}$ with the hadronic decay of the top quark and primarily the $\mathrm{b} \overline{\mathrm{b}}$ decay of the Higgs and $\mathrm{Z}$ bosons. This search is designed to be sensitive to $\mathrm{T}$ quark fractional widths of up to $30 \%$ and a wide range of masses. The background is mostly due to standard model $t \bar{t}+$ jets and QCD multijet events with some contributions from $\mathrm{W}+$ jets processes. No significant excess of data above the standard model background is observed and upper limits at $95 \%$ confidence level are set on $\sigma \mathcal{B}(\mathrm{T} \rightarrow \mathrm{tH})$ and $\sigma \mathcal{B}(\mathrm{T} \rightarrow \mathrm{tZ})$, which vary between $2 \mathrm{pb}$ and $20 \mathrm{fb}$ for $\mathrm{T}$ masses ranging from 0.6 to $2.6 \mathrm{TeV}$ in the $\mathrm{Tbq}$ and $\mathrm{Ttq}$ production channels. Results from 

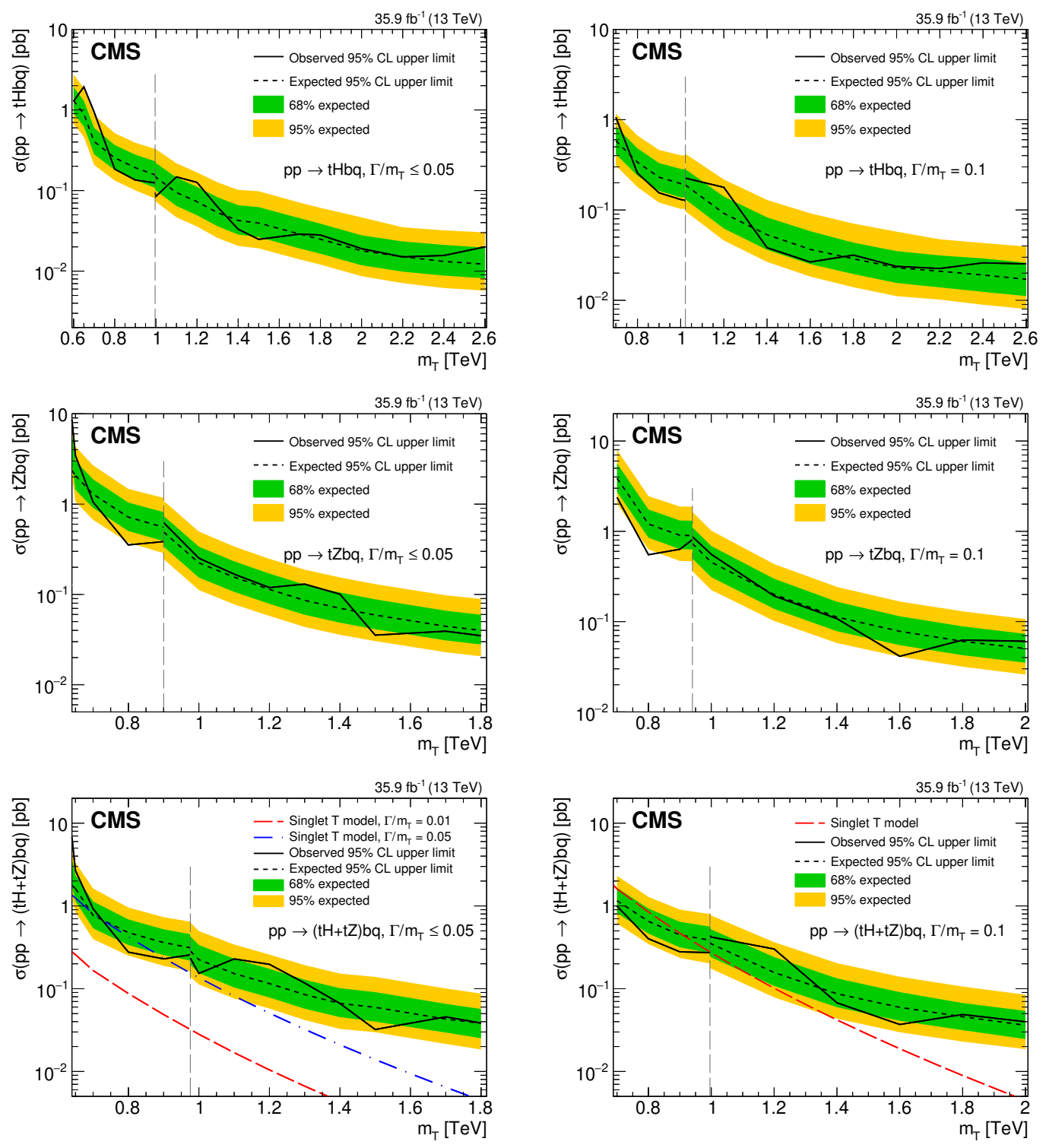

Figure 8. The observed and median expected upper limits at 95\% CL on the cross sections for production associated with a bottom quark for the tHbq (upper row) and tZbq (middle row) channels, and their sum, tHbq + tZbq (lower row), for different assumed values of the $\mathrm{T}$ quark mass. The inner (green) band and the outer (yellow) band indicate the regions containing 68 and $95 \%$, respectively, of the distribution of limits expected under the background-only hypothesis. The left column is for a narrow fractional width $\left(\Gamma / m_{\mathrm{T}} \leq 0.05\right)$ and the right column is for a fractional width of $\Gamma / m_{\mathrm{T}}=0.1$. The vertical dashed lines are the crossover points in sensitivity that indicate the mass intervals used for presenting the low-mass and high-mass search results. The dashed red and dot-dashed blue curves are for the $\mathrm{T}$ singlet model. Given the specified width, the couplings are implicit in the model. Two curves corresponding to $\Gamma / m_{\mathrm{T}}=0.05$ (dot-dashed blue) and $\Gamma / m_{\mathrm{T}}=0.01$ (dashed red) are shown for the narrow fractional width. 

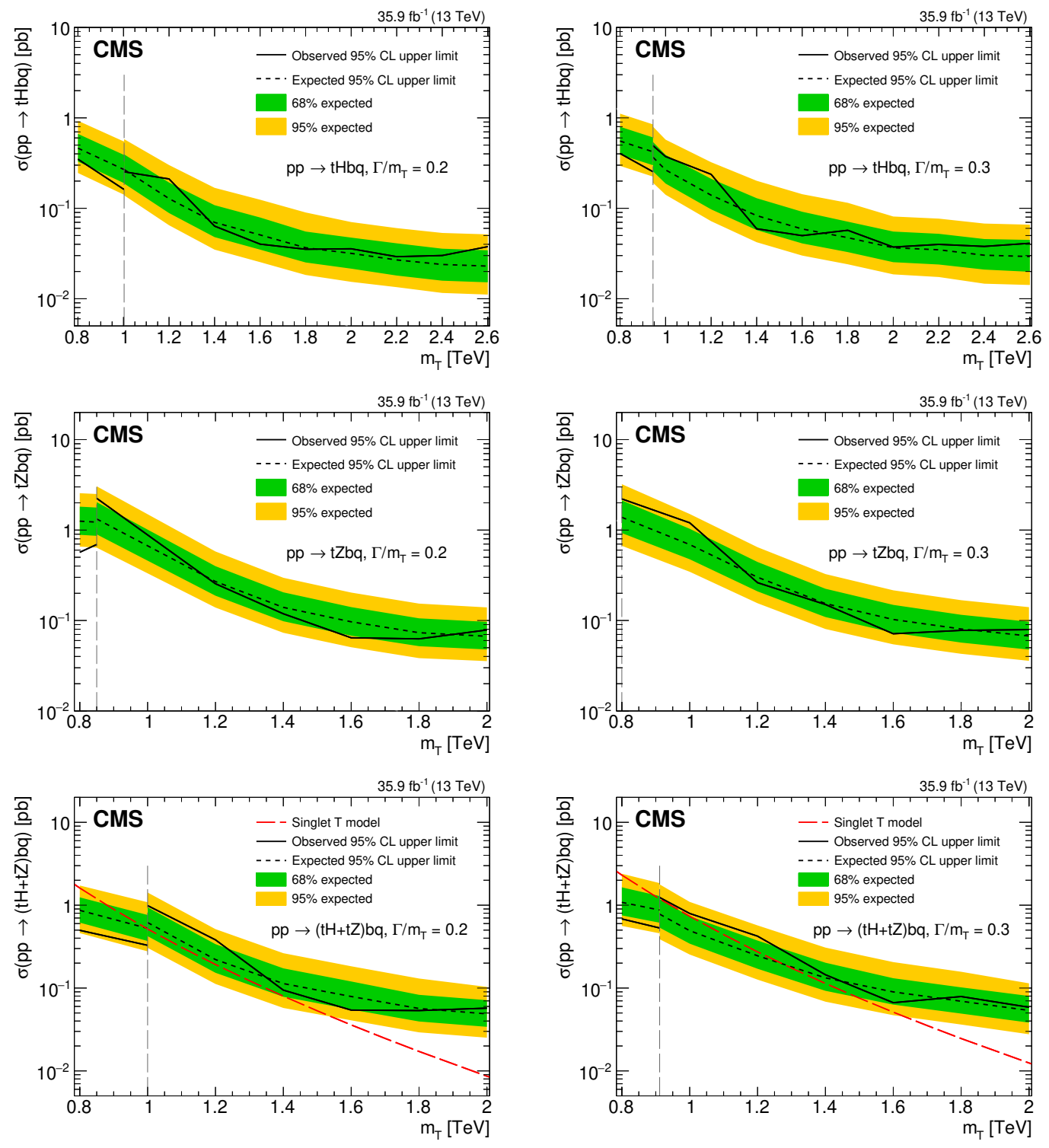

Figure 9. The observed and median expected upper limits at 95\% CL on the cross sections for production associated with a bottom quark for the tHbq (upper row) and tZbq (middle row) channels, and their sum, tHbq + tZbq (lower row), for different assumed values of the $\mathrm{T}$ quark mass. The inner (green) band and the outer (yellow) band indicate the regions containing 68 and $95 \%$, respectively, of the distribution of limits expected under the background-only hypothesis. The left column is for a fractional width of $20 \%$ and the right column is for a fractional width of $30 \%$. The vertical dashed lines are the crossover points in sensitivity that indicate the mass intervals used for presenting the low-mass and high-mass search results. The dashed red curves are for the $\mathrm{T}$ singlet model. Given the specified width, the couplings are implicit in the model. 

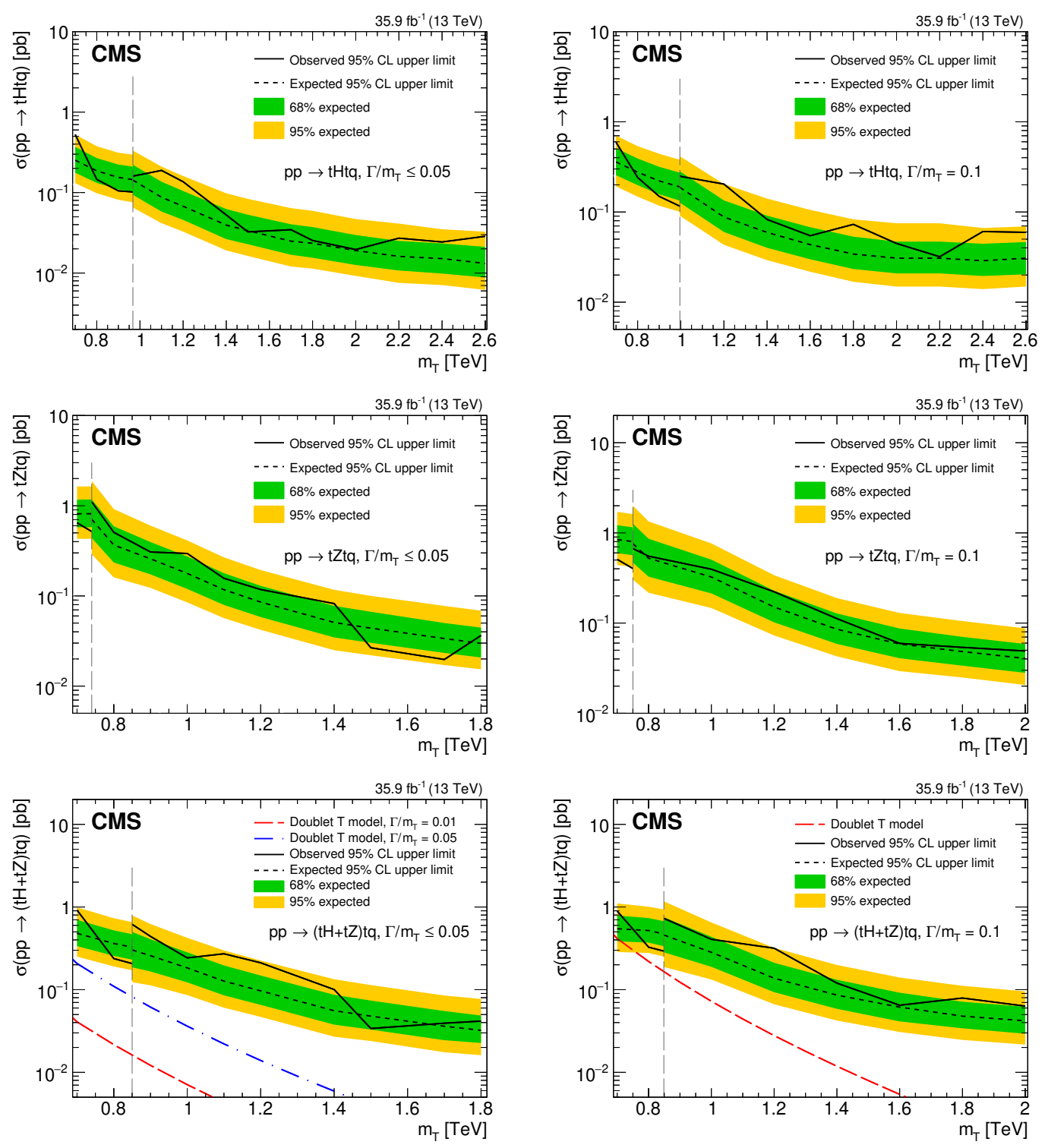

Figure 10. The observed and median expected upper limits at 95\% CL on the cross sections for production associated with a top quark for the tHtq (upper row) and tZtq (middle row) channels, and their sum, tHtq + tZtq (lower row), for different assumed values of the T quark mass. The inner (green) band and the outer (yellow) band indicate the regions containing 68 and 95\%, respectively, of the distribution of limits expected under the background-only hypothesis. The left column is for a narrow fractional width $\left(\Gamma / m_{\mathrm{T}} \leq 0.05\right)$ and the right column is for a fractional width of $\Gamma / m_{\mathrm{T}}=0.1$. The vertical dashed lines are the crossover points in sensitivity that indicate the mass intervals used for presenting the low-mass and high-mass search results. The dashed red and dot-dashed blue curves are for the (TB) doublet model. Given the specified width, the couplings are implicit in the model. Two curves corresponding to $\Gamma / m_{\mathrm{T}}=0.05$ (dot-dashed blue) and $\Gamma / m_{\mathrm{T}}=0.01$ (dashed red) are shown for the narrow fractional width. 

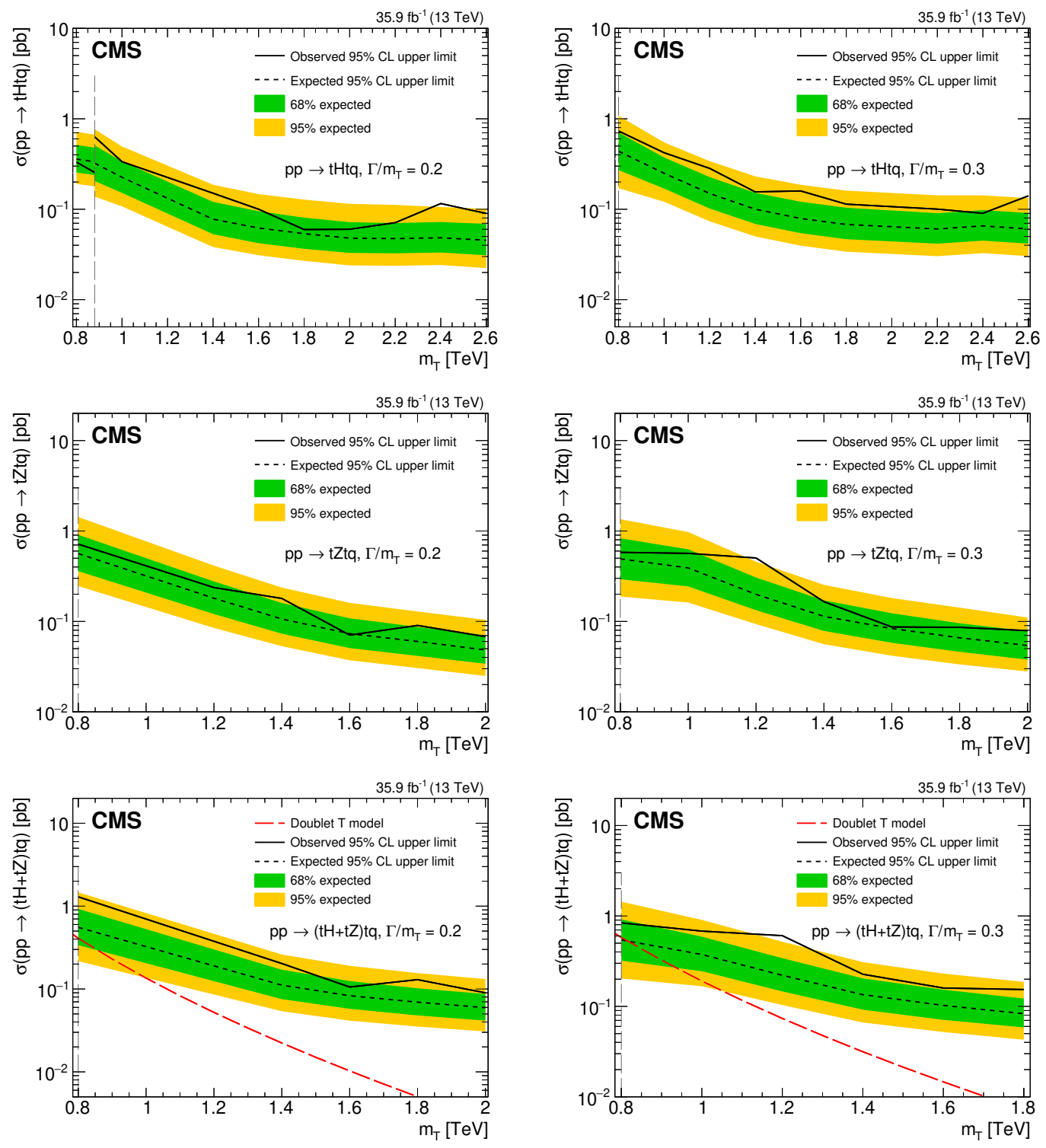

Figure 11. The observed and median expected upper limits at 95\% CL on the cross sections for production associated with a top quark for the tHtq (upper row) and tZtq (middle row) channels, and their sum, tHtq + tZtq (lower row), for different assumed values of the T quark mass. The inner (green) band and the outer (yellow) band indicate the regions containing 68 and 95\%, respectively, of the distribution of limits expected under the background-only hypothesis. The left column is for a fractional width of $20 \%$ and the right column is for a fractional width of $30 \%$. The vertical dashed lines are the crossover points in sensitivity that indicate the mass intervals used for presenting the low-mass and high-mass search results. The dashed red curves are for the (TB) doublet model. Given the specified width, the couplings are implicit in the model. 
combining the two decay channels assuming equal couplings are also reported. Compared with prior electroweak single production searches, this search is significantly more sensitive for $\mathrm{T} \rightarrow \mathrm{tH}$. The search gives the first constraints using this production mode on $\mathrm{T} \rightarrow \mathrm{tZ}$ for hadronic decays of the $\mathrm{Z}$ boson. These results are competitive with those from searches for $\mathrm{T} \rightarrow \mathrm{tZ}$ using other $\mathrm{Z}$ decay modes. The combined $\mathrm{T} \rightarrow \mathrm{tH}$ and $\mathrm{T} \rightarrow \mathrm{tZ}$ results for associated production with a bottom quark lead to constraints on $\mathrm{T}$ quarks in the $\mathrm{T}$ singlet model for masses below $1.00 \mathrm{TeV}$. The expected sensitivity for this model extends to $1.28 \mathrm{TeV}$ (for $30 \%$ fractional width), which is comparable to the mass reach of the most stringent pair production searches.

\section{Acknowledgments}

We congratulate our colleagues in the CERN accelerator departments for the excellent performance of the LHC and thank the technical and administrative staffs at CERN and at other CMS institutes for their contributions to the success of the CMS effort. In addition, we gratefully acknowledge the computing centers and personnel of the Worldwide LHC Computing Grid for delivering so effectively the computing infrastructure essential to our analyses. Finally, we acknowledge the enduring support for the construction and operation of the LHC and the CMS detector provided by the following funding agencies: BMBWF and FWF (Austria); FNRS and FWO (Belgium); CNPq, CAPES, FAPERJ, FAPERGS, and FAPESP (Brazil); MES (Bulgaria); CERN; CAS, MoST, and NSFC (China); COLCIENCIAS (Colombia); MSES and CSF (Croatia); RPF (Cyprus); SENESCYT (Ecuador); MoER, ERC IUT, PUT and ERDF (Estonia); Academy of Finland, MEC, and HIP (Finland); CEA and CNRS/IN2P3 (France); BMBF, DFG, and HGF (Germany); GSRT (Greece); NKFIA (Hungary); DAE and DST (India); IPM (Iran); SFI (Ireland); INFN (Italy); MSIP and NRF (Republic of Korea); MES (Latvia); LAS (Lithuania); MOE and UM (Malaysia); BUAP, CINVESTAV, CONACYT, LNS, SEP, and UASLP-FAI (Mexico); MOS (Montenegro); MBIE (New Zealand); PAEC (Pakistan); MSHE and NSC (Poland); FCT (Portugal); JINR (Dubna); MON, RosAtom, RAS, RFBR, and NRC KI (Russia); MESTD (Serbia); SEIDI, CPAN, PCTI, and FEDER (Spain); MOSTR (Sri Lanka); Swiss Funding Agencies (Switzerland); MST (Taipei); ThEPCenter, IPST, STAR, and NSTDA (Thailand); TUBITAK and TAEK (Turkey); NASU and SFFR (Ukraine); STFC (United Kingdom); DOE and NSF (U.S.A.).

Individuals have received support from the Marie-Curie program and the European Research Council and Horizon 2020 Grant, contract Nos. 675440, 752730, and 765710 (European Union); the Leventis Foundation; the A.P. Sloan Foundation; the Alexander von Humboldt Foundation; the Belgian Federal Science Policy Office; the Fonds pour la Formation à la Recherche dans l'Industrie et dans l'Agriculture (FRIA-Belgium); the Agentschap voor Innovatie door Wetenschap en Technologie (IWT-Belgium); the F.R.S.-FNRS and FWO (Belgium) under the "Excellence of Science — EOS" — be.h project n. 30820817; the Beijing Municipal Science \& Technology Commission, No. Z181100004218003; the Ministry of Education, Youth and Sports (MEYS) of the Czech Republic; the Lendület ("Momentum") Program and the János Bolyai Research Scholarship of the Hungarian 
Academy of Sciences, the New National Excellence Program ÚNKP, the NKFIA research grants 123842, 123959, 124845, 124850, 125105, 128713, 128786, and 129058 (Hungary); the Council of Science and Industrial Research, India; the HOMING PLUS program of the Foundation for Polish Science, cofinanced from European Union, Regional Development Fund, the Mobility Plus program of the Ministry of Science and Higher Education, the National Science Center (Poland), contracts Harmonia 2014/14/M/ST2/00428, Opus 2014/13/B/ST2/02543, 2014/15/B/ST2/03998, and 2015/19/B/ST2/02861, Sonatabis 2012/07/E/ST2/01406; the National Priorities Research Program by Qatar National Research Fund; the Ministry of Science and Education, grant no. 3.2989.2017 (Russia); the Programa Estatal de Fomento de la Investigación Científica y Técnica de Excelencia María de Maeztu, grant MDM-2015-0509 and the Programa Severo Ochoa del Principado de Asturias; the Thalis and Aristeia programs cofinanced by EU-ESF and the Greek NSRF; the Rachadapisek Sompot Fund for Postdoctoral Fellowship, Chulalongkorn University and the Chulalongkorn Academic into Its 2nd Century Project Advancement Project (Thailand); the Nvidia Corporation; the Welch Foundation, contract C-1845; and the Weston Havens Foundation (U.S.A.). 


\section{A Low-mass and high-mass search limits}

For information, we show here separately the limits obtained with each of the two search signatures that were used to give the final search limit results presented in figures 8-11 of the paper.
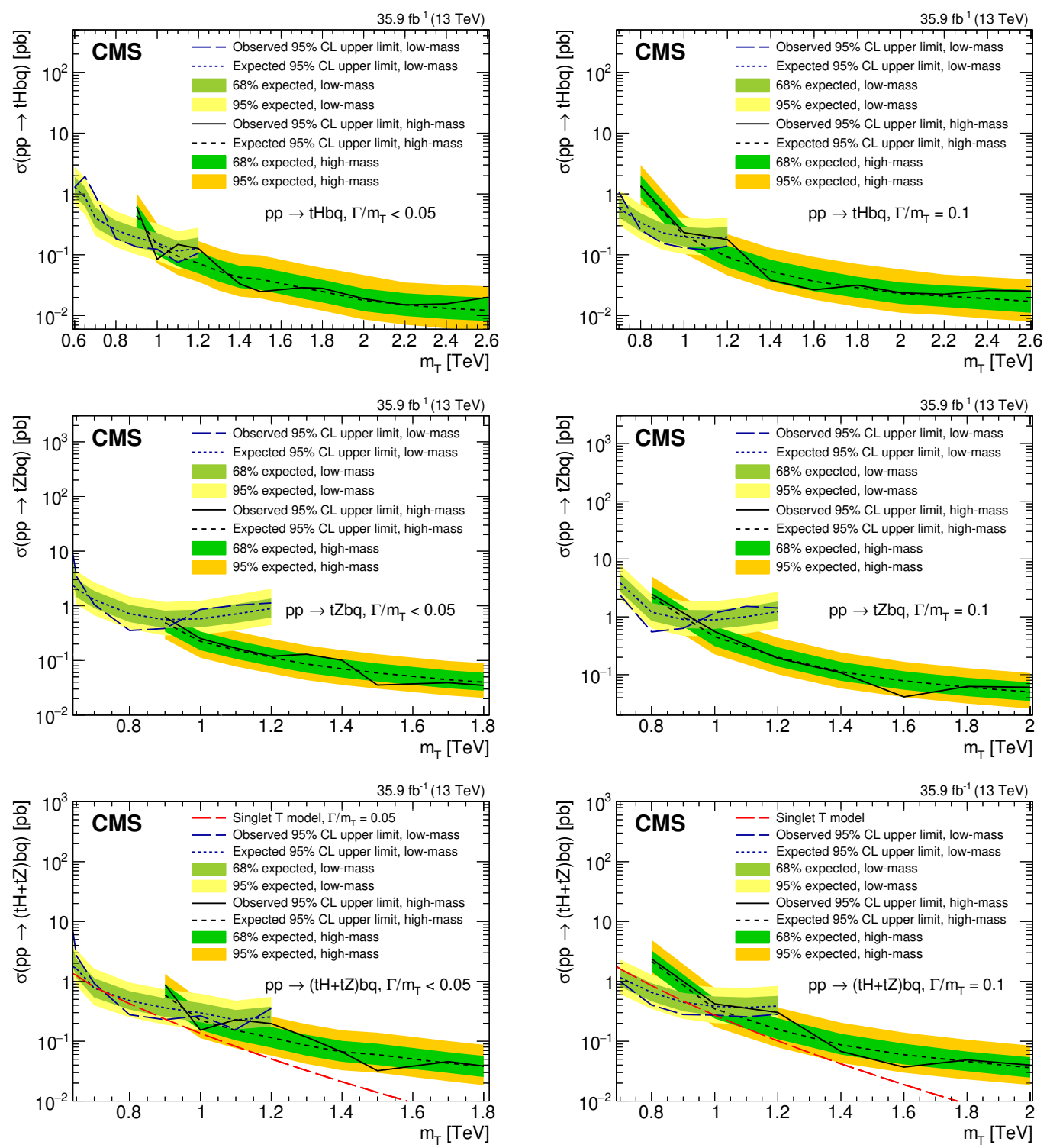

Figure 12. The observed and median expected upper limits at 95\% CL on the cross sections for production associated with a bottom quark for the tHbq (upper row) and tZbq (middle row) channels, and their sum, tHbq + tZbq (lower row), for different assumed values of the T quark mass. The inner (green) bands and the outer (yellow) bands indicate the regions containing 68 and $95 \%$, respectively, of the distribution of limits expected under the background-only hypothesis. The left column is for a narrow fractional width $\left(\Gamma / m_{\mathrm{T}} \leq 0.05\right)$ and the right column is for a fractional width of $\Gamma / m_{\mathrm{T}}=0.1$. The dashed red curves are for the $\mathrm{T}$ singlet model. Given the specified width, the couplings are implicit in the model. 

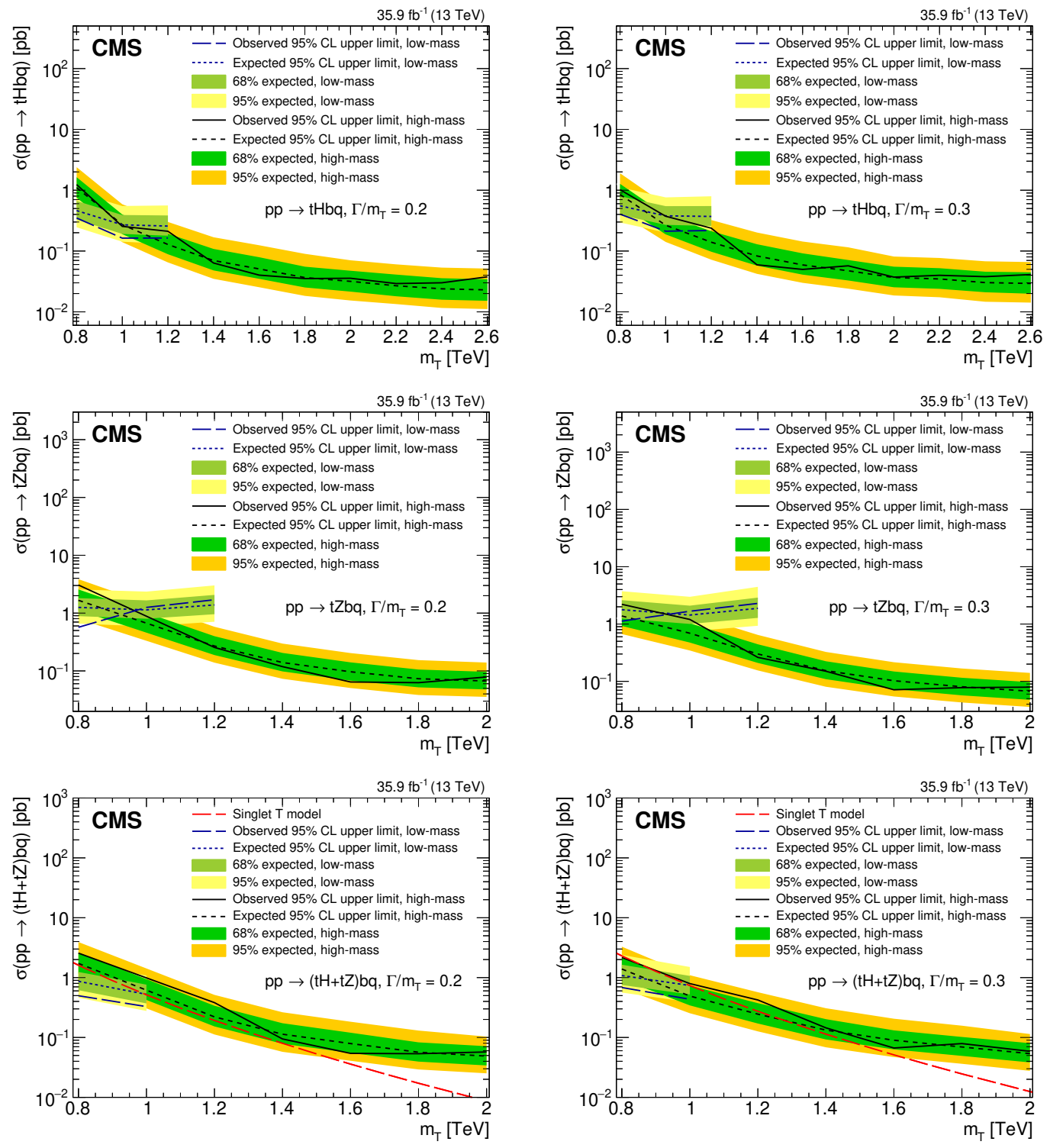

Figure 13. The observed and median expected upper limits at 95\% CL on the cross sections for production associated with a bottom quark for the tHbq (upper row) and tZbq (middle row) channels, and their sum, tHbq + tZbq (lower row), for different assumed values of the $\mathrm{T}$ quark mass. The inner (green) bands and the outer (yellow) bands indicate the regions containing 68 and $95 \%$, respectively, of the distribution of limits expected under the background-only hypothesis. The left column is for a fractional width of $20 \%$ and the right column is for a fractional width of $30 \%$. The dashed red curves are for the $\mathrm{T}$ singlet model. Given the specified width, the couplings are implicit in the model. 

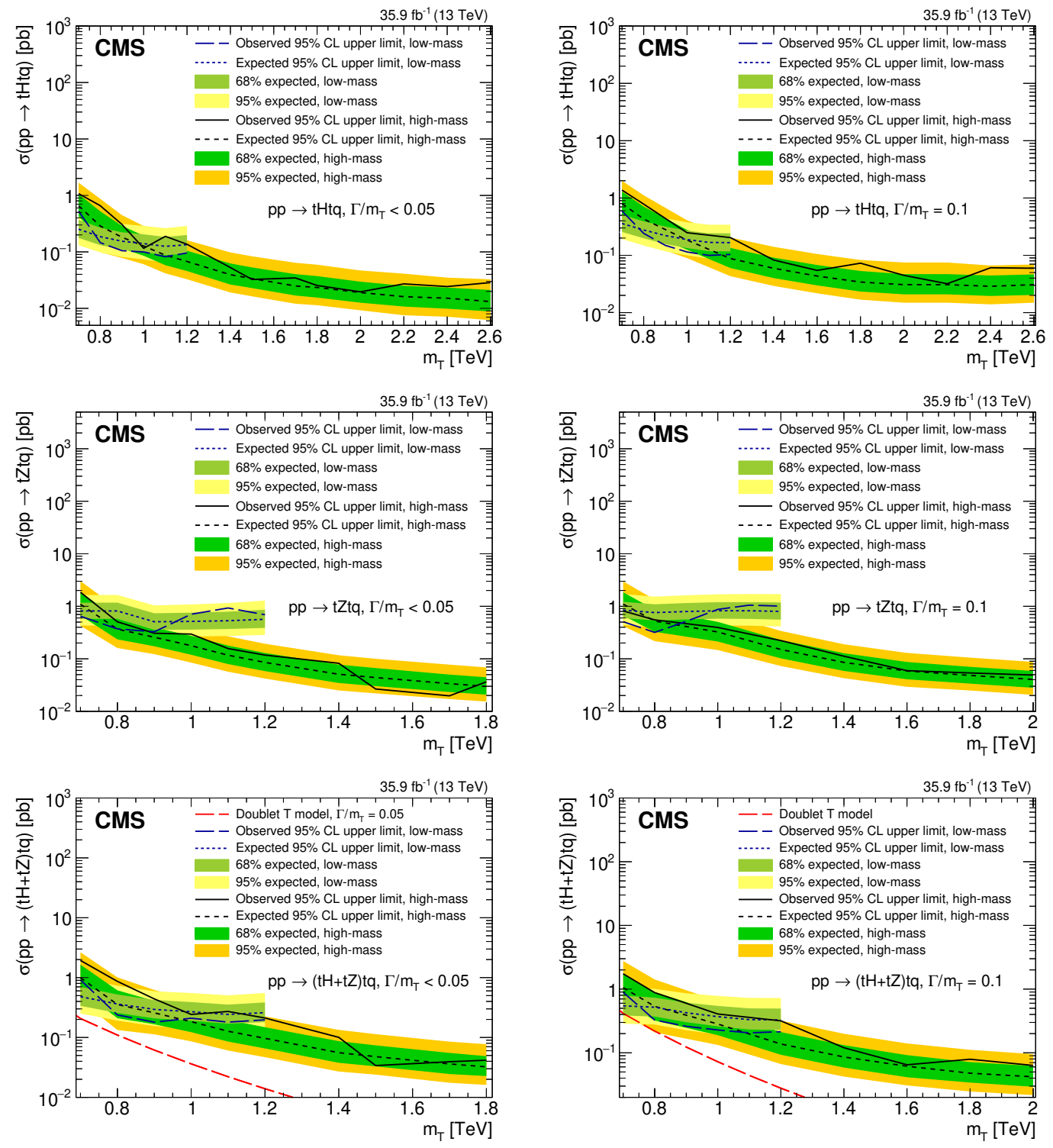

Figure 14. The observed and median expected upper limits at 95\% CL on the cross sections for production associated with a top quark for the tHtq (upper row) and tZtq (middle row) channels, and their sum, tHtq + tZtq (lower row), for different assumed values of the T quark mass. The inner (green) bands and the outer (yellow) bands indicate the regions containing 68 and 95\%, respectively, of the distribution of limits expected under the background-only hypothesis. The left column is for a narrow fractional width $\left(\Gamma / m_{\mathrm{T}} \leq 0.05\right)$ and the right column is for a fractional width of $\Gamma / m_{\mathrm{T}}=0.1$. The dashed red curves are for the (TB) doublet model. Given the specified width, the couplings are implicit in the model. 

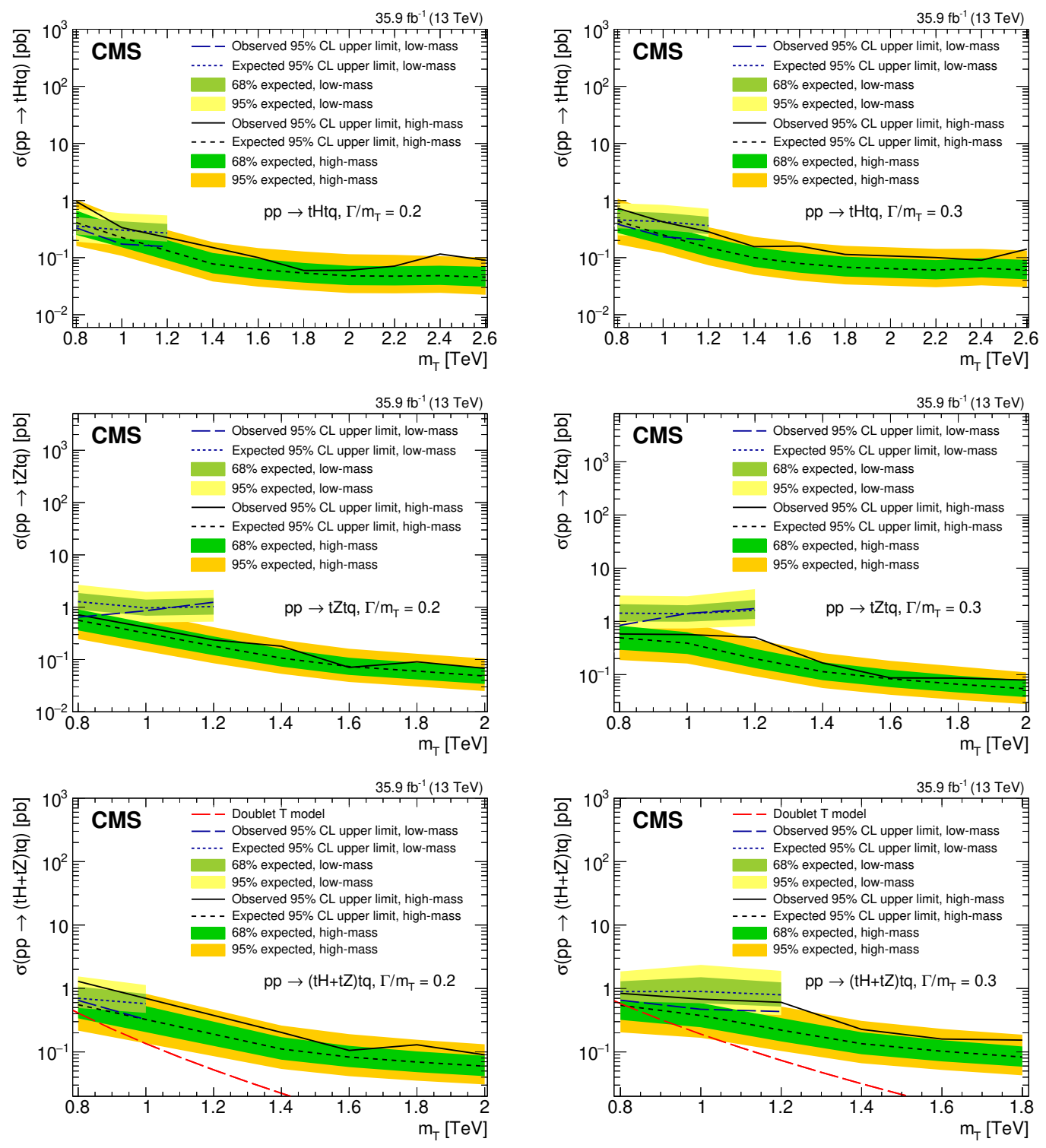

Figure 15. The observed and median expected upper limits at 95\% CL on the cross sections for production associated with a top quark for the tHtq (upper row) and tZtq (middle row) channels, and their sum, tHtq + tZtq (lower row), for different assumed values of the T quark mass. The inner (green) bands and the outer (yellow) bands indicate the regions containing 68 and 95\%, respectively, of the distribution of limits expected under the background-only hypothesis. The left column is for a fractional width of $20 \%$ and the right column is for a fractional width of $30 \%$. The dashed red curves are for the (TB) doublet model. Given the specified width, the couplings are implicit in the model. 
Open Access. This article is distributed under the terms of the Creative Commons Attribution License (CC-BY 4.0), which permits any use, distribution and reproduction in any medium, provided the original author(s) and source are credited.

\section{References}

[1] Y. Okada and L. Panizzi, LHC signatures of vector-like quarks, Adv. High Energy Phys. 2013 (2013) 364936 [arXiv:1207.5607] [INSPIRE].

[2] J.A. Aguilar-Saavedra, R. Benbrik, S. Heinemeyer and M. Pérez-Victoria, Handbook of vectorlike quarks: Mixing and single production, Phys. Rev. D 88 (2013) 094010 [arXiv: 1306.0572] [INSPIRE].

[3] A. De Simone, O. Matsedonskyi, R. Rattazzi and A. Wulzer, A first top partner hunter's guide, JHEP 04 (2013) 004 [arXiv:1211.5663] [INSPIRE].

[4] M. Buchkremer, G. Cacciapaglia, A. Deandrea and L. Panizzi, Model independent framework for searches of top partners, Nucl. Phys. B 876 (2013) 376 [arXiv:1305.4172] [InSPIRE].

[5] J.A. Aguilar-Saavedra, Identifying top partners at LHC, JHEP 11 (2009) 030 [arXiv: 0907.3155] [INSPIRE].

[6] B.A. Dobrescu, K. Kong and R. Mahbubani, Prospects for top-prime quark discovery at the Tevatron, JHEP 06 (2009) 001 [arXiv: 0902.0792] [INSPIRE].

[7] N. Vignaroli, Early discovery of top partners and test of the Higgs nature, Phys. Rev. D 86 (2012) 075017 [arXiv: 1207.0830] [INSPIRE].

[8] T. Han, H.E. Logan, B. McElrath and L.-T. Wang, Phenomenology of the little Higgs model, Phys. Rev. D 67 (2003) 095004 [hep-ph/0301040] [INSPIRE].

[9] ATLAS collaboration, Search for pair production of vector-like top quarks in events with one lepton, jets and missing transverse momentum in $\sqrt{s}=13 \mathrm{TeV} p p$ collisions with the ATLAS detector, JHEP 08 (2017) 052 [arXiv:1705.10751] [INSPIRE].

[10] CMS collaboration, Search for pair production of vector-like $T$ and $B$ quarks in single-lepton final states using boosted jet substructure in proton-proton collisions at $\sqrt{s}=13 \mathrm{TeV}$, JHEP 11 (2017) 085 [arXiv: 1706. 03408] [INSPIRE].

[11] ATLAS collaboration, Search for pair production of heavy vector-like quarks decaying to high-p $p_{T} W$ bosons and $b$ quarks in the lepton-plus-jets final state in pp collisions at $\sqrt{s}=13$ TeV with the ATLAS detector, JHEP 10 (2017) 141 [arXiv:1707.03347] [INSPIRE].

[12] CMS collaboration, Search for pair production of vector-like quarks in the $b W \bar{b} W$ channel from proton-proton collisions at $\sqrt{s}=13 \mathrm{TeV}$, Phys. Lett. B 779 (2018) 82 [arXiv: 1710.01539] [INSPIRE].

[13] ATLAS collaboration, Search for pair production of up-type vector-like quarks and for four-top-quark events in final states with multiple b-jets with the ATLAS detector, JHEP 07 (2018) 089 [arXiv : 1803.09678] [INSPIRE].

[14] CMS collaboration, Search for vector-like $T$ and $B$ quark pairs in final states with leptons at $\sqrt{s}=13 \mathrm{TeV}$, JHEP 08 (2018) 177 [arXiv: 1805.04758] [INSPIRE].

[15] ATLAS collaboration, Search for pair- and single-production of vector-like quarks in final states with at least one $Z$ boson decaying into a pair of electrons or muons in pp collision data collected with the ATLAS detector at $\sqrt{s}=13 \mathrm{TeV}$, Phys. Rev. D 98 (2018) 112010 [arXiv: 1806.10555] [INSPIRE]. 
[16] ATLAS collaboration, Combination of the searches for pair-produced vector-like partners of the third-generation quarks at $\sqrt{s}=13$ TeV with the ATLAS detector, Phys. Rev. Lett. 121 (2018) 211801 [arXiv: 1808.02343] [INSPIRE].

[17] CMS collaboration, Search for vector-like quarks in events with two oppositely charged leptons and jets in proton-proton collisions at $\sqrt{s}=13$ TeV, Eur. Phys. J. C 79 (2019) 364 [arXiv: 1812.09768] [INSPIRE].

[18] CMS collaboration, Search for pair production of vectorlike quarks in the fully hadronic final state, Phys. Rev. D 100 (2019) 072001 [arXiv: 1906.11903] [InSPIRE].

[19] ATLAS collaboration, Search for pair and single production of new heavy quarks that decay to a $Z$ boson and a third-generation quark in pp collisions at $\sqrt{s}=8$ TeV with the ATLAS detector, JHEP 11 (2014) 104 [arXiv:1409.5500] [INSPIRE].

[20] CMS collaboration, Search for single production of a heavy vector-like $T$ quark decaying to a Higgs boson and a top quark with a lepton and jets in the final state, Phys. Lett. $\mathbf{B} 771$ (2017) 80 [arXiv: 1612.00999] [INSPIRE].

[21] CMS collaboration, Search for electroweak production of a vector-like quark decaying to a top quark and a Higgs boson using boosted topologies in fully hadronic final states, JHEP 04 (2017) 136 [arXiv:1612.05336] [INSPIRE].

[22] CMS collaboration, Search for single production of vector-like quarks decaying into a $b$ quark and a $W$ boson in proton-proton collisions at $\sqrt{s}=13$ TeV, Phys. Lett. B 772 (2017) 634 [arXiv: 1701.08328] [INSPIRE].

[23] ATLAS collaboration, Search for single production of vector-like quarks decaying into Wb in pp collisions at $\sqrt{s}=13$ TeV with the ATLAS detector, JHEP 05 (2019) 164 [arXiv: 1812.07343] [INSPIRE].

[24] CMS collaboration, Search for single production of vector-like quarks decaying to a $Z$ boson and a top or a bottom quark in proton-proton collisions at $\sqrt{s}=13$ TeV, JHEP 05 (2017) 029 [arXiv: 1701.07409] [INSPIRE].

[25] CMS collaboration, Search for single production of a vector-like $T$ quark decaying to a $Z$ boson and a top quark in proton-proton collisions at $\sqrt{s}=13$ TeV, Phys. Lett. B 781 (2018) 574 [arXiv: 1708.01062] [INSPIRE].

[26] ATLAS collaboration, Search for large missing transverse momentum in association with one top-quark in proton-proton collisions at $\sqrt{s}=13$ TeV with the ATLAS detector, JHEP 05 (2019) 041 [arXiv: 1812.09743] [INSPIRE].

[27] CMS collaboration, Description and performance of track and primary-vertex reconstruction with the CMS tracker, 2014 JINST 9 P10009 [arXiv:1405.6569] [INSPIRE].

[28] CMS collaboration, The CMS trigger system, 2017 JINST 12 P01020 [arXiv:1609.02366] [INSPIRE].

[29] M. Cacciari, G.P. Salam and G. Soyez, The anti- $k_{t}$ jet clustering algorithm, JHEP 04 (2008) 063 [arXiv:0802.1189] [INSPIRE].

[30] M. Cacciari, G.P. Salam and G. Soyez, FastJet user manual, Eur. Phys. J. C 72 (2012) 1896 [arXiv:1111.6097] [INSPIRE].

[31] CMS collaboration, Particle-flow reconstruction and global event description with the CMS detector, 2017 JINST 12 P10003 [arXiv:1706.04965] [INSPIRE].

[32] CMS collaboration, Jet energy scale and resolution in the CMS experiment in pp collisions at $8 \mathrm{TeV}, 2017$ JINST 12 P02014 [arXiv: 1607.03663] [INSPIRE]. 
[33] CMS collaboration, The CMS experiment at the CERN LHC, 2008 JINST 3 S08004 [INSPIRE].

[34] J. Alwall et al., The automated computation of tree-level and next-to-leading order differential cross sections and their matching to parton shower simulations, JHEP 07 (2014) 079 [arXiv: 1405.0301] [INSPIRE].

[35] NNPDF collaboration, Parton distributions for the LHC Run II, JHEP 04 (2015) 040 [arXiv: 1410.8849] [INSPIRE].

[36] T. Sjöstrand, S. Mrenna and P.Z. Skands, A brief introduction to PYTHIA 8.1, Comput. Phys. Commun. 178 (2008) 852 [arXiv:0710.3820] [INSPIRE].

[37] CMS collaboration, Investigations of the impact of the parton shower tuning in PYTHIA 8 in the modelling of $t \bar{t}$ at $\sqrt{s}=8$ and 13 TeV, CMS-PAS-TOP-16-021 (2016).

[38] CMS collaboration, Event generator tunes obtained from underlying event and multiparton scattering measurements, Eur. Phys. J. C 76 (2016) 155 [arXiv:1512.00815] [INSPIRE].

[39] P. Nason, A new method for combining NLO QCD with shower Monte Carlo algorithms, JHEP 11 (2004) 040 [hep-ph/0409146] [INSPIRE].

[40] S. Frixione, P. Nason and C. Oleari, Matching NLO QCD computations with Parton Shower simulations: the POWHEG method, JHEP 11 (2007) 070 [arXiv:0709.2092] [INSPIRE].

[41] S. Alioli, P. Nason, C. Oleari and E. Re, A general framework for implementing NLO calculations in shower Monte Carlo programs: the POWHEG BOX, JHEP 06 (2010) 043 [arXiv: 1002.2581] [INSPIRE].

[42] S. Frixione, P. Nason and G. Ridolfi, A positive-weight next-to-leading-order Monte Carlo for heavy flavour hadroproduction, JHEP 09 (2007) 126 [arXiv:0707.3088] [INSPIRE].

[43] P. Artoisenet, R. Frederix, O. Mattelaer and R. Rietkerk, Automatic spin-entangled decays of heavy resonances in Monte Carlo simulations, JHEP 03 (2013) 015 [arXiv:1212.3460] [INSPIRE].

[44] R. Frederix and S. Frixione, Merging meets matching in MC@NLO, JHEP 12 (2012) 061 [arXiv: 1209.6215] [INSPIRE].

[45] J. Alwall et al., Comparative study of various algorithms for the merging of parton showers and matrix elements in hadronic collisions, Eur. Phys. J. C 53 (2008) 473 [arXiv:0706.2569] [INSPIRE].

[46] R.D. Ball et al., Parton distributions with LHC data, Nucl. Phys. B 867 (2013) 244 [arXiv: 1207.1303] [INSPIRE].

[47] CMS collaboration, Identification of heavy-flavour jets with the CMS detector in pp collisions at $13 \mathrm{TeV}, 2018$ JINST 13 P05011 [arXiv:1712.07158] [INSPIRE].

[48] A.J. Larkoski, S. Marzani, G. Soyez and J. Thaler, Soft drop, JHEP 05 (2014) 146 [arXiv: 1402.2657] [INSPIRE].

[49] Particle Data Group collaboration, Review of particle physics, Phys. Rev. D 98 (2018) 030001 [INSPIRE].

[50] G. Punzi, Sensitivity of searches for new signals and its optimization, eConf C 030908 (2003) MODT002 [physics/0308063] [INSPIRE].

[51] T. Junk, Confidence level computation for combining searches with small statistics, Nucl. Instrum. Meth. A 434 (1999) 435 [hep-ex/9902006] [INSPIRE].

[52] A.L. Read, Presentation of search results: the CL technique, J. Phys. G 28 (2002) 2693 [INSPIRE]. 
[53] G. Cowan, K. Cranmer, E. Gross and O. Vitells, Asymptotic formulae for likelihood-based tests of new physics, Eur. Phys. J. C 71 (2011) 1554 [Erratum ibid. C 73 (2013) 2501] [arXiv: 1007.1727] [INSPIRE].

[54] G.P. Salam, Towards jetography, Eur. Phys. J. C 67 (2010) 637 [arXiv:0906.1833] [INSPIRE].

[55] M. Dasgupta, A. Fregoso, S. Marzani and G.P. Salam, Towards an understanding of jet substructure, JHEP 09 (2013) 029 [arXiv:1307.0007] [INSPIRE].

[56] S.D. Ellis, C.K. Vermilion and J.R. Walsh, Recombination algorithms and jet substructure: pruning as a tool for heavy particle searches, Phys. Rev. D 81 (2010) 094023 [arXiv: 0912.0033] [INSPIRE].

[57] J. Thaler and K. Van Tilburg, Identifying boosted objects with $N$-subjettiness, JHEP 03 (2011) 015 [arXiv: 1011.2268] [INSPIRE].

[58] CMS collaboration, Top tagging with new approaches, CMS-PAS-JME-15-002 (2015).

[59] CMS collaboration, Search for Higgs boson pair production in the $\gamma \gamma b \bar{b}$ final state in $p p$ collisions at $\sqrt{s}=13 \mathrm{TeV}$, Phys. Lett. B 788 (2019) 7 [arXiv: 1806.00408] [INSPIRE].

[60] N. Kumar and S.P. Martin, LHC search for di-Higgs decays of stoponium and other scalars in events with two photons and two bottom jets, Phys. Rev. D 90 (2014) 055007 [arXiv: 1404.0996] [INSPIRE].

[61] CMS collaboration, Jet algorithms performance in $13 \mathrm{TeV}$ data, CMS-PAS-JME-16-003 (2016).

[62] M. Bahr et al., HERWIG++ physics and manual, Eur. Phys. J. C 58 (2008) 639 [arXiv: 0803.0883] [INSPIRE].

[63] S. Gieseke, C. Rohr and A. Siodmok, Colour reconnections in HERWIG++, Eur. Phys. J. C 72 (2012) 2225 [arXiv:1206.0041] [INSPIRE].

[64] J. Butterworth et al., PDF4LHC recommendations for LHC Run II, J. Phys. G 43 (2016) 023001 [arXiv: 1510.03865] [INSPIRE].

[65] CMS collaboration, CMS luminosity measurements for the 2016 data taking period, CMS-PAS-LUM-17-001 (2017).

[66] CMS collaboration, Measurement of the inelastic proton-proton cross section at $\sqrt{s}=13$ TeV, JHEP 07 (2018) 161 [arXiv: 1802.02613] [INSPIRE].

[67] M. Czakon, P. Fiedler and A. Mitov, Total top-quark pair-production cross section at hadron colliders through $O\left(\alpha_{s}^{4}\right)$, Phys. Rev. Lett. 110 (2013) 252004 [arXiv:1303.6254] [InSPIRE].

[68] K. Melnikov and F. Petriello, Electroweak gauge boson production at hadron colliders through $O\left(\alpha_{s}^{2}\right)$, Phys. Rev. D 74 (2006) 114017 [hep-ph/0609070] [INSPIRE].

[69] Y. Li and F. Petriello, Combining QCD and electroweak corrections to dilepton production in FEWZ, Phys. Rev. D 86 (2012) 094034 [arXiv:1208.5967] [INSPIRE].

[70] A. Carvalho et al., Single production of vectorlike quarks with large width at the Large Hadron Collider, Phys. Rev. D 98 (2018) 015029 [arXiv:1805.06402] [INSPIRE]. 


\section{The CMS collaboration}

Yerevan Physics Institute, Yerevan, Armenia

A.M. Sirunyan ${ }^{\dagger}$, A. Tumasyan

\section{Institut für Hochenergiephysik, Wien, Austria}

W. Adam, F. Ambrogi, T. Bergauer, J. Brandstetter, M. Dragicevic, J. Erö, A. Escalante Del Valle, M. Flechl, R. Frühwirth ${ }^{1}$, M. Jeitler ${ }^{1}$, N. Krammer, I. Krätschmer, D. Liko, T. Madlener, I. Mikulec, N. Rad, J. Schieck ${ }^{1}$, R. Schöfbeck, M. Spanring, D. Spitzbart, W. Waltenberger, C.-E. Wulz ${ }^{1}$, M. Zarucki

Institute for Nuclear Problems, Minsk, Belarus

V. Drugakov, V. Mossolov, J. Suarez Gonzalez

\section{Universiteit Antwerpen, Antwerpen, Belgium}

M.R. Darwish, E.A. De Wolf, D. Di Croce, X. Janssen, A. Lelek, M. Pieters, H. Rejeb Sfar, H. Van Haevermaet, P. Van Mechelen, S. Van Putte, N. Van Remortel

\section{Vrije Universiteit Brussel, Brussel, Belgium}

F. Blekman, E.S. Bols, S.S. Chhibra, J. D'Hondt, J. De Clercq, D. Lontkovskyi, S. Lowette, I. Marchesini, S. Moortgat, Q. Python, K. Skovpen, S. Tavernier, W. Van Doninck, P. Van Mulders

\section{Université Libre de Bruxelles, Bruxelles, Belgium}

D. Beghin, B. Bilin, H. Brun, B. Clerbaux, G. De Lentdecker, H. Delannoy, B. Dorney, L. Favart, A. Grebenyuk, A.K. Kalsi, A. Popov, N. Postiau, E. Starling, L. Thomas, C. Vander Velde, P. Vanlaer, D. Vannerom

\section{Ghent University, Ghent, Belgium}

T. Cornelis, D. Dobur, I. Khvastunov ${ }^{2}$, M. Niedziela, C. Roskas, D. Trocino, M. Tytgat, W. Verbeke, B. Vermassen, M. Vit, N. Zaganidis

Université Catholique de Louvain, Louvain-la-Neuve, Belgium

O. Bondu, G. Bruno, C. Caputo, P. David, C. Delaere, M. Delcourt, A. Giammanco, V. Lemaitre, A. Magitteri, J. Prisciandaro, A. Saggio, M. Vidal Marono, P. Vischia, J. Zobec

Centro Brasileiro de Pesquisas Fisicas, Rio de Janeiro, Brazil

F.L. Alves, G.A. Alves, G. Correia Silva, C. Hensel, A. Moraes, P. Rebello Teles

Universidade do Estado do Rio de Janeiro, Rio de Janeiro, Brazil

E. Belchior Batista Das Chagas, W. Carvalho, J. Chinellato ${ }^{3}$, E. Coelho, E.M. Da Costa, G.G. Da Silveira ${ }^{4}$, D. De Jesus Damiao, C. De Oliveira Martins, S. Fonseca De Souza, L.M. Huertas Guativa, H. Malbouisson, J. Martins ${ }^{5}$, D. Matos Figueiredo, M. Medina Jaime ${ }^{6}$, M. Melo De Almeida, C. Mora Herrera, L. Mundim, H. Nogima, W.L. Prado Da Silva, L.J. Sanchez Rosas, A. Santoro, A. Sznajder, M. Thiel, E.J. Tonelli Manganote ${ }^{3}$, F. Torres Da Silva De Araujo, A. Vilela Pereira 
Universidade Estadual Paulista ${ }^{a}$, Universidade Federal do $\mathrm{ABC}^{b}$, São Paulo, Brazil

C.A. Bernardes ${ }^{a}$, L. Calligaris ${ }^{a}$, T.R. Fernandez Perez Tomei ${ }^{a}$, E.M. Gregores ${ }^{b}$, D.S. Lemos, P.G. Mercadante ${ }^{b}$, S.F. Novaes ${ }^{a}$, SandraS. Padula ${ }^{a}$

Institute for Nuclear Research and Nuclear Energy, Bulgarian Academy of Sciences, Sofia, Bulgaria

A. Aleksandrov, G. Antchev, R. Hadjiiska, P. Iaydjiev, M. Misheva, M. Rodozov, M. Shopova, G. Sultanov

University of Sofia, Sofia, Bulgaria

M. Bonchev, A. Dimitrov, T. Ivanov, L. Litov, B. Pavlov, P. Petkov

Beihang University, Beijing, China

W. Fang ${ }^{7}, \mathrm{X} . \mathrm{Gao}^{7}, \mathrm{~L}$. Yuan

Institute of High Energy Physics, Beijing, China

M. Ahmad, G.M. Chen, H.S. Chen, M. Chen, C.H. Jiang, D. Leggat, H. Liao, Z. Liu, S.M. Shaheen ${ }^{8}$, A. Spiezia, J. Tao, E. Yazgan, H. Zhang, S. Zhang ${ }^{8}$, J. Zhao

State Key Laboratory of Nuclear Physics and Technology, Peking University, Beijing, China

A. Agapitos, Y. Ban, G. Chen, A. Levin, J. Li, L. Li, Q. Li, Y. Mao, S.J. Qian, D. Wang, Q. Wang

Tsinghua University, Beijing, China

Z. Hu, Y. Wang

Zhejiang University, Hangzhou, China

M. Xiao

Universidad de Los Andes, Bogota, Colombia

C. Avila, A. Cabrera, C. Florez, C.F. González Hernández, M.A. Segura Delgado

Universidad de Antioquia, Medellin, Colombia

J. Mejia Guisao, J.D. Ruiz Alvarez, C.A. Salazar González, N. Vanegas Arbelaez

University of Split, Faculty of Electrical Engineering, Mechanical Engineering and Naval Architecture, Split, Croatia

D. Giljanović, N. Godinovic, D. Lelas, I. Puljak, T. Sculac

University of Split, Faculty of Science, Split, Croatia

Z. Antunovic, M. Kovac

Institute Rudjer Boskovic, Zagreb, Croatia

V. Brigljevic, S. Ceci, D. Ferencek, K. Kadija, B. Mesic, M. Roguljic, A. Starodumov ${ }^{9}$, T. Susa 
University of Cyprus, Nicosia, Cyprus

M.W. Ather, A. Attikis, E. Erodotou, A. Ioannou, M. Kolosova, S. Konstantinou, G. Mavromanolakis, J. Mousa, C. Nicolaou, F. Ptochos, P.A. Razis, H. Rykaczewski, D. Tsiakkouri

Charles University, Prague, Czech Republic

M. Finger ${ }^{10}$, M. Finger Jr. ${ }^{10}$, A. Kveton, J. Tomsa

Escuela Politecnica Nacional, Quito, Ecuador

E. Ayala

Universidad San Francisco de Quito, Quito, Ecuador

E. Carrera Jarrin

Academy of Scientific Research and Technology of the Arab Republic of Egypt, Egyptian Network of High Energy Physics, Cairo, Egypt

S. Elgammal ${ }^{11}$, E. Salama ${ }^{11,12}$

National Institute of Chemical Physics and Biophysics, Tallinn, Estonia

S. Bhowmik, A. Carvalho Antunes De Oliveira, R.K. Dewanjee, K. Ehataht, M. Kadastik, M. Raidal, C. Veelken

Department of Physics, University of Helsinki, Helsinki, Finland

P. Eerola, L. Forthomme, H. Kirschenmann, K. Osterberg, M. Voutilainen

Helsinki Institute of Physics, Helsinki, Finland

F. Garcia, J. Havukainen, J.K. Heikkilä, T. Järvinen, V. Karimäki, M.S. Kim, R. Kinnunen,

T. Lampén, K. Lassila-Perini, S. Laurila, S. Lehti, T. Lindén, P. Luukka, T. Mäenpää,

H. Siikonen, E. Tuominen, J. Tuominiemi

Lappeenranta University of Technology, Lappeenranta, Finland

T. Tuuva

IRFU, CEA, Université Paris-Saclay, Gif-sur-Yvette, France

M. Besancon, F. Couderc, M. Dejardin, D. Denegri, B. Fabbro, J.L. Faure, F. Ferri, S. Ganjour, A. Givernaud, P. Gras, G. Hamel de Monchenault, P. Jarry, C. Leloup, E. Locci, J. Malcles, J. Rander, A. Rosowsky, M.Ö. Sahin, A. Savoy-Navarro ${ }^{13}$, M. Titov

Laboratoire Leprince-Ringuet, Ecole polytechnique, CNRS/IN2P3, Université Paris-Saclay, Palaiseau, France

S. Ahuja, C. Amendola, F. Beaudette, P. Busson, C. Charlot, B. Diab, G. Falmagne,

R. Granier de Cassagnac, I. Kucher, A. Lobanov, C. Martin Perez, M. Nguyen, C. Ochando,

P. Paganini, J. Rembser, R. Salerno, J.B. Sauvan, Y. Sirois, A. Zabi, A. Zghiche

Université de Strasbourg, CNRS, IPHC UMR 7178, Strasbourg, France

J.-L. Agram ${ }^{14}$, J. Andrea, D. Bloch, G. Bourgatte, J.-M. Brom, E.C. Chabert, C. Collard,

E. Conte ${ }^{14}$, J.-C. Fontaine ${ }^{14}$, D. Gelé, U. Goerlach, M. Jansová, A.-C. Le Bihan, N. Tonon, P. Van Hove 
Centre de Calcul de l'Institut National de Physique Nucleaire et de Physique des Particules, CNRS/IN2P3, Villeurbanne, France

S. Gadrat

Université de Lyon, Université Claude Bernard Lyon 1, CNRS-IN2P3, Institut de Physique Nucléaire de Lyon, Villeurbanne, France

S. Beauceron, C. Bernet, G. Boudoul, C. Camen, A. Carle, N. Chanon, R. Chierici, D. Contardo, P. Depasse, H. El Mamouni, J. Fay, S. Gascon, M. Gouzevitch, B. Ille, Sa. Jain, F. Lagarde, I.B. Laktineh, H. Lattaud, A. Lesauvage, M. Lethuillier, L. Mirabito, S. Perries, V. Sordini, L. Torterotot, G. Touquet, M. Vander Donckt, S. Viret

Georgian Technical University, Tbilisi, Georgia

T. Toriashvili ${ }^{15}$

Tbilisi State University, Tbilisi, Georgia

Z. Tsamalaidze ${ }^{10}$

RWTH Aachen University, I. Physikalisches Institut, Aachen, Germany

C. Autermann, L. Feld, M.K. Kiesel, K. Klein, M. Lipinski, D. Meuser, A. Pauls, M. Preuten, M.P. Rauch, C. Schomakers, J. Schulz, M. Teroerde, B. Wittmer

RWTH Aachen University, III. Physikalisches Institut A, Aachen, Germany

A. Albert, M. Erdmann, B. Fischer, S. Ghosh, T. Hebbeker, K. Hoepfner, H. Keller, L. Mastrolorenzo, M. Merschmeyer, A. Meyer, P. Millet, G. Mocellin, S. Mondal, S. Mukherjee, D. Noll, A. Novak, T. Pook, A. Pozdnyakov, T. Quast, M. Radziej, Y. Rath, H. Reithler, J. Roemer, A. Schmidt, S.C. Schuler, A. Sharma, S. Wiedenbeck, S. Zaleski

RWTH Aachen University, III. Physikalisches Institut B, Aachen, Germany

G. Flügge, W. Haj Ahmad ${ }^{16}$, O. Hlushchenko, T. Kress, T. Müller, A. Nehrkorn, A. Nowack, C. Pistone, O. Pooth, D. Roy, H. Sert, A. Stahl ${ }^{17}$

Deutsches Elektronen-Synchrotron, Hamburg, Germany

M. Aldaya Martin, P. Asmuss, I. Babounikau, H. Bakhshiansohi, K. Beernaert, O. Behnke, A. Bermúdez Martínez, D. Bertsche, A.A. Bin Anuar, K. Borras ${ }^{18}$, V. Botta, A. Campbell, A. Cardini, P. Connor, S. Consuegra Rodríguez, C. Contreras-Campana, V. Danilov, A. De Wit, M.M. Defranchis, C. Diez Pardos, D. Domínguez Damiani, G. Eckerlin, D. Eckstein, T. Eichhorn, A. Elwood, E. Eren, E. Gallo ${ }^{19}$, A. Geiser, A. Grohsjean, M. Guthoff, M. Haranko, A. Harb, A. Jafari, N.Z. Jomhari, H. Jung, A. Kasem ${ }^{18}$, M. Kasemann, H. Kaveh, J. Keaveney, C. Kleinwort, J. Knolle, D. Krücker, W. Lange, T. Lenz, J. Leonard, J. Lidrych, K. Lipka, W. Lohmann ${ }^{20}$, R. Mankel, I.-A. Melzer-Pellmann, A.B. Meyer, M. Meyer, M. Missiroli, G. Mittag, J. Mnich, A. Mussgiller, V. Myronenko, D. Pérez Adán, S.K. Pflitsch, D. Pitzl, A. Raspereza, A. Saibel, M. Savitskyi, V. Scheurer, P. Schütze, C. Schwanenberger, R. Shevchenko, A. Singh, H. Tholen, O. Turkot, A. Vagnerini, M. Van De Klundert, R. Walsh, Y. Wen, K. Wichmann, C. Wissing, O. Zenaiev, R. Zlebcik University of Hamburg, Hamburg, Germany

R. Aggleton, S. Bein, L. Benato, A. Benecke, V. Blobel, T. Dreyer, A. Ebrahimi, F. Feindt, A. Fröhlich, C. Garbers, E. Garutti, D. Gonzalez, P. Gunnellini, J. Haller, A. Hinzmann, 
A. Karavdina, G. Kasieczka, R. Klanner, R. Kogler, N. Kovalchuk, S. Kurz, V. Kutzner, J. Lange, T. Lange, A. Malara, J. Multhaup, C.E.N. Niemeyer, A. Perieanu, A. Reimers, O. Rieger, C. Scharf, P. Schleper, S. Schumann, J. Schwandt, J. Sonneveld, H. Stadie, G. Steinbrück, F.M. Stober, M. Stöver, B. Vormwald, I. Zoi

Karlsruher Institut fuer Technologie, Karlsruhe, Germany

M. Akbiyik, C. Barth, M. Baselga, S. Baur, T. Berger, E. Butz, R. Caspart, T. Chwalek, W. De Boer, A. Dierlamm, K. El Morabit, N. Faltermann, M. Giffels, P. Goldenzweig, A. Gottmann, M.A. Harrendorf, F. Hartmann ${ }^{17}$, U. Husemann, S. Kudella, S. Mitra, M.U. Mozer, D. Müller, Th. Müller, M. Musich, A. Nürnberg, G. Quast, K. Rabbertz, M. Schröder, I. Shvetsov, H.J. Simonis, R. Ulrich, M. Wassmer, M. Weber, C. Wöhrmann, R. Wolf

Institute of Nuclear and Particle Physics (INPP), NCSR Demokritos, Aghia Paraskevi, Greece

G. Anagnostou, P. Asenov, G. Daskalakis, T. Geralis, A. Kyriakis, D. Loukas, G. Paspalaki

National and Kapodistrian University of Athens, Athens, Greece

M. Diamantopoulou, G. Karathanasis, P. Kontaxakis, A. Manousakis-katsikakis, A. Panagiotou, I. Papavergou, N. Saoulidou, A. Stakia, K. Theofilatos, K. Vellidis, E. Vourliotis

National Technical University of Athens, Athens, Greece

G. Bakas, K. Kousouris, I. Papakrivopoulos, G. Tsipolitis

University of Ioánnina, Ioánnina, Greece

I. Evangelou, C. Foudas, P. Gianneios, P. Katsoulis, P. Kokkas, S. Mallios, K. Manitara, N. Manthos, I. Papadopoulos, J. Strologas, F.A. Triantis, D. Tsitsonis

MTA-ELTE Lendület CMS Particle and Nuclear Physics Group, Eötvös Loránd University, Budapest, Hungary

M. Bartók ${ }^{21}$, R. Chudasama, M. Csanad, P. Major, K. Mandal, A. Mehta, M.I. Nagy, G. Pasztor, O. Surányi, G.I. Veres

Wigner Research Centre for Physics, Budapest, Hungary

G. Bencze, C. Hajdu, D. Horvath ${ }^{22}$, F. Sikler, T.Á. Vámi, V. Veszpremi, G. Vesztergombi ${ }^{\dagger}$

Institute of Nuclear Research ATOMKI, Debrecen, Hungary

N. Beni, S. Czellar, J. Karancsi ${ }^{21}$, A. Makovec, J. Molnar, Z. Szillasi

Institute of Physics, University of Debrecen, Debrecen, Hungary

P. Raics, D. Teyssier, Z.L. Trocsanyi, B. Ujvari

Eszterhazy Karoly University, Karoly Robert Campus, Gyongyos, Hungary

T. Csorgo, W.J. Metzger, F. Nemes, T. Novak

Indian Institute of Science (IISc), Bangalore, India

S. Choudhury, J.R. Komaragiri, P.C. Tiwari 
National Institute of Science Education and Research, HBNI, Bhubaneswar, India

S. Bahinipati ${ }^{24}$, C. Kar, G. Kole, P. Mal, V.K. Muraleedharan Nair Bindhu, A. Nayak ${ }^{25}$, D.K. Sahoo ${ }^{24}$, S.K. Swain

Panjab University, Chandigarh, India

S. Bansal, S.B. Beri, V. Bhatnagar, S. Chauhan, R. Chawla, N. Dhingra, R. Gupta,

A. Kaur, M. Kaur, S. Kaur, P. Kumari, M. Lohan, M. Meena, K. Sandeep, S. Sharma, J.B. Singh, A.K. Virdi, G. Walia

University of Delhi, Delhi, India

A. Bhardwaj, B.C. Choudhary, R.B. Garg, M. Gola, S. Keshri, Ashok Kumar, S. Malhotra, M. Naimuddin, P. Priyanka, K. Ranjan, Aashaq Shah, R. Sharma

Saha Institute of Nuclear Physics, HBNI, Kolkata, India

R. Bhardwaj ${ }^{26}$, M. Bharti ${ }^{26}$, R. Bhattacharya, S. Bhattacharya, U. Bhawandeep ${ }^{26}$, D. Bhowmik, S. Dutta, S. Ghosh, M. Maity ${ }^{27}$, K. Mondal, S. Nandan, A. Purohit, P.K. Rout, G. Saha, S. Sarkar, T. Sarkar ${ }^{27}$, M. Sharan, B. Singh ${ }^{26}$, S. Thakur ${ }^{26}$

Indian Institute of Technology Madras, Madras, India

P.K. Behera, P. Kalbhor, A. Muhammad, P.R. Pujahari, A. Sharma, A.K. Sikdar

Bhabha Atomic Research Centre, Mumbai, India

D. Dutta, V. Jha, V. Kumar, D.K. Mishra, P.K. Netrakanti, L.M. Pant, P. Shukla

Tata Institute of Fundamental Research-A, Mumbai, India

T. Aziz, M.A. Bhat, S. Dugad, G.B. Mohanty, N. Sur, RavindraKumar Verma

Tata Institute of Fundamental Research-B, Mumbai, India

S. Banerjee, S. Bhattacharya, S. Chatterjee, P. Das, M. Guchait, S. Karmakar, S. Kumar,

G. Majumder, K. Mazumdar, N. Sahoo, S. Sawant

Indian Institute of Science Education and Research (IISER), Pune, India

S. Chauhan, S. Dube, V. Hegde, B. Kansal, A. Kapoor, K. Kothekar, S. Pandey, A. Rane, A. Rastogi, S. Sharma

Institute for Research in Fundamental Sciences (IPM), Tehran, Iran

S. Chenarani ${ }^{28}$, E. Eskandari Tadavani, S.M. Etesami ${ }^{28}$, M. Khakzad, M. Mohammadi Najafabadi, M. Naseri, F. Rezaei Hosseinabadi

University College Dublin, Dublin, Ireland

M. Felcini, M. Grunewald

INFN Sezione di Bari ${ }^{a}$, Università di Bari ${ }^{b}$, Politecnico di Bari ${ }^{c}$, Bari, Italy

M. Abbrescia ${ }^{a, b}$, R. Aly ${ }^{a, b, 29}$, C. Calabria ${ }^{a, b}$, A. Colaleo $^{a}$, D. Creanza $^{a, c}$, L. Cristella $^{a, b}$, N. De Filippis ${ }^{a, c}$, M. De Palma ${ }^{a, b}$, A. Di Florio ${ }^{a, b}$, L. Fiore $^{a}$, A. Gelmi $^{a, b}$, G. Iaselli ${ }^{a, c}$, M. Ince ${ }^{a, b}$, S. Lezki ${ }^{a, b}$, G. Maggi ${ }^{a, c}$, M. Maggi ${ }^{a}$, G. Miniello ${ }^{a, b}$, S. My ${ }^{a, b}$, S. Nuzzo ${ }^{a, b}$, A. Pompili ${ }^{a, b}$, G. Pugliese ${ }^{a, c}$, R. Radogna ${ }^{a}$, A. Ranieri ${ }^{a}$, G. Selvaggi ${ }^{a, b}$, L. Silvestris ${ }^{a}$,

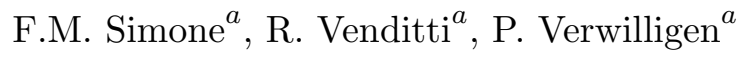


INFN Sezione di Bologna ${ }^{a}$, Università di Bologna ${ }^{b}$, Bologna, Italy

G. Abbiendi ${ }^{a}$, C. Battilana ${ }^{a, b}$, D. Bonacorsi ${ }^{a, b}$, L. Borgonovi $^{a, b}$, S. Braibant-Giacomelli $^{a, b}$, R. Campanini ${ }^{a, b}$, P. Capiluppi ${ }^{a, b}$, A. Castro ${ }^{a, b}$, F.R. Cavallo ${ }^{a}$, C. Ciocca $^{a}$, G. Codispoti $^{a, b}{ }^{,}$, M. Cuffiani ${ }^{a, b}$, G.M. Dallavalle ${ }^{a}$, F. Fabbri ${ }^{a}$, A. Fanfani ${ }^{a, b}$, E. Fontanesi ${ }^{a, b}$, P. Giacomelli ${ }^{a}$, C. Grandi ${ }^{a}$, L. Guiducci ${ }^{a, b}$, F. Iemmi ${ }^{a, b}$, S. Lo Meo ${ }^{a, 30}$, S. Marcellini ${ }^{a}$, G. Masetti ${ }^{a}$,

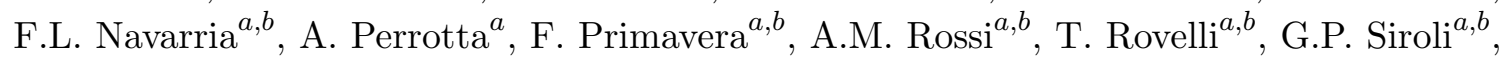
N. $\operatorname{Tosi}^{a}$

INFN Sezione di Catania ${ }^{a}$, Università di Catania ${ }^{b}$, Catania, Italy

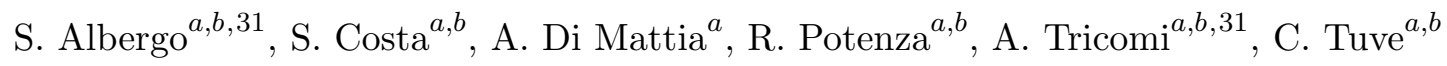

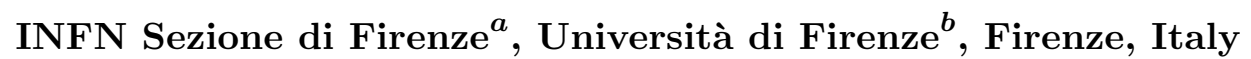

G. Barbagli ${ }^{a}$, A. Cassese, R. Ceccarelli, K. Chatterjee ${ }^{a, b}$, V. Ciulli ${ }^{a, b}$, C. Civinini ${ }^{a}$, R. D’Alessandro ${ }^{a, b}$, E. Focardi ${ }^{a, b}$, G. Latino ${ }^{a, b}$, P. Lenzi $^{a, b}$, M. Meschini ${ }^{a}$, S. Paoletti $^{a}$, G. Sguazzoni ${ }^{a}$, L. Viliani ${ }^{a}$

INFN Laboratori Nazionali di Frascati, Frascati, Italy

L. Benussi, S. Bianco, D. Piccolo

INFN Sezione di Genova ${ }^{a}$, Università di Genova ${ }^{b}$, Genova, Italy

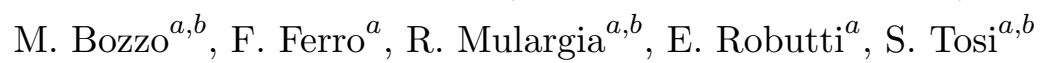

INFN Sezione di Milano-Bicocca $^{a}$, Università di Milano-Bicocca ${ }^{b}$, Milano, Italy

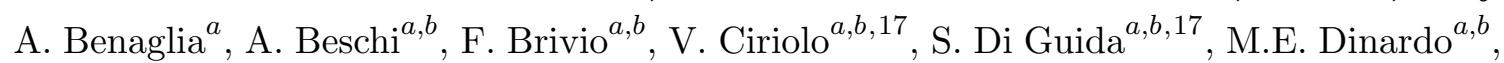
P. Dini ${ }^{a}$, S. Gennai ${ }^{a}$, A. Ghezzi ${ }^{a, b}$, P. Govoni ${ }^{a, b}$, L. Guzzi $^{a} b$, M. Malberti ${ }^{a}$, S. Malvezzi ${ }^{a}$, D. Menasce ${ }^{a}$, F. Monti ${ }^{a, b}$, L. Moroni ${ }^{a}$, M. Paganoni ${ }^{a, b}$, D. Pedrini ${ }^{a}$, S. Ragazzi ${ }^{a, b}$, T. Tabarelli de Fatis ${ }^{a, b}$, D. Zuolo ${ }^{a, b}$

INFN Sezione di Napoli ${ }^{a}$, Università di Napoli 'Federico II ${ }^{b}$, Napoli, Italy, Università della Basilicata $^{c}$, Potenza, Italy, Università G. Marconi ${ }^{d}$, Roma, Italy

S. Buontempo ${ }^{a}$, N. Cavallo $^{a, c}$, A. De Iorio ${ }^{a, b}$, A. Di Crescenzo ${ }^{a, b}$, F. Fabozzi $^{a, c}$, F. Fienga $^{a}$,

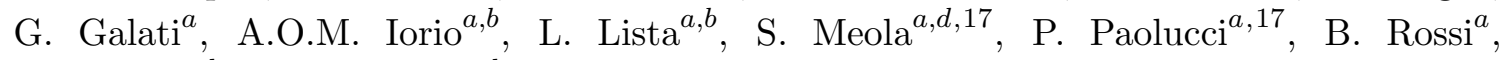
C. Sciacca ${ }^{a, b}$, E. Voevodina ${ }^{a, b}$

INFN Sezione di Padova ${ }^{a}$, Università di Padova ${ }^{b}$, Padova, Italy, Università di Trento $^{c}$, Trento, Italy

P. $\operatorname{Azzi}^{a}$, N. Bacchetta ${ }^{a}$, D. Bisello ${ }^{a, b}$, A. Boletti ${ }^{a, b}$, A. Bragagnolo ${ }^{a, b}$, R. Carlin ${ }^{a, b}$, P. Checchia ${ }^{a}$, P. De Castro Manzano ${ }^{a}$, T. Dorigo ${ }^{a}$, U. Dosselli ${ }^{a}$, F. Gasparini ${ }^{a, b}$, U. Gasparini $^{a, b}$, A. Gozzelino ${ }^{a}$, S.Y. Hoh ${ }^{a, b}$, P. Lujan ${ }^{a}$, M. Margoni $^{a, b}$, A.T. Meneguzzo $^{a, b}$, J. Pazzini ${ }^{a, b}$, M. Presilla ${ }^{b}$, P. Ronchese ${ }^{a, b}$, R. Rossin ${ }^{a, b}$, F. Simonetto ${ }^{a, b}$, A. Tiko ${ }^{a}$, M. Tosi ${ }^{a, b}$, M. Zanetti ${ }^{a, b}$, P. Zotto ${ }^{a, b}$, G. Zumerle ${ }^{a, b}$

INFN Sezione di Pavia ${ }^{a}$, Università di Pavia ${ }^{b}$, Pavia, Italy

A. Braghieri ${ }^{a}$, D. Fiorina ${ }^{a, b}$, P. Montagna ${ }^{a, b}$, S.P. Ratti ${ }^{a, b}$, V. Re ${ }^{a}$, M. Ressegotti $^{a, b}$,

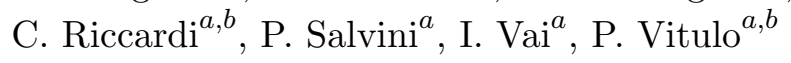


INFN Sezione di Perugia ${ }^{a}$, Università di Perugia ${ }^{b}$, Perugia, Italy M. Biasini ${ }^{a, b}$, G.M. Bilei ${ }^{a}$, D. Ciangottini ${ }^{a, b}$, L. Fanò ${ }^{a, b}$, P. Lariccia ${ }^{a, b}$, R. Leonardi ${ }^{a, b}$, G. Mantovani ${ }^{a, b}$, V. Mariani ${ }^{a, b}$, M. Menichelli ${ }^{a}$, A. Rossi ${ }^{a, b}$, A. Santocchia $^{a, b}$, D. Spiga $^{a}$

INFN Sezione di Pisa ${ }^{a}$, Università di Pisa ${ }^{b}$, Scuola Normale Superiore di Pisa ${ }^{c}$, Pisa, Italy

K. Androsov $^{a}$, P. Azzurri ${ }^{a}$, G. Bagliesi ${ }^{a}$, V. Bertacchi ${ }^{a, c}$, L. Bianchini $^{a}$, T. Boccali ${ }^{a}$,

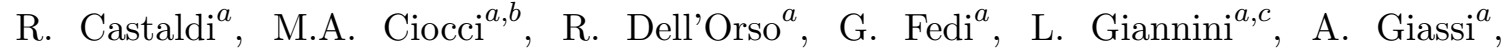
M.T. Grippo ${ }^{a}$, F. Ligabue ${ }^{a, c}$, E. Manca ${ }^{a, c}$, G. Mandorli ${ }^{a, c}$, A. Messineo ${ }^{a, b}$, F. Palla $^{a}$, A. Rizzi ${ }^{a, b}$, G. Rolandi ${ }^{32}$, S. Roy Chowdhury, A. Scribano ${ }^{a}$, P. Spagnolo $^{a}$, R. Tenchini ${ }^{a}$, G. Tonelli ${ }^{a, b}$, N. Turini, A. Venturi ${ }^{a}$, P.G. Verdini ${ }^{a}$

INFN Sezione di Roma ${ }^{a}$, Sapienza Università di Roma ${ }^{b}$, Rome, Italy

F. Cavallari ${ }^{a}$, M. Cipriani ${ }^{a, b}$, D. Del Re ${ }^{a, b}$, E. Di Marco ${ }^{a, b}$, M. Diemoz $^{a}$, E. Longo ${ }^{a, b}$, B. Marzocchi ${ }^{a, b}$, P. Meridiani ${ }^{a}$, G. Organtini ${ }^{a, b}$, F. Pandolfi ${ }^{a}$, R. Paramatti ${ }^{a, b}$,

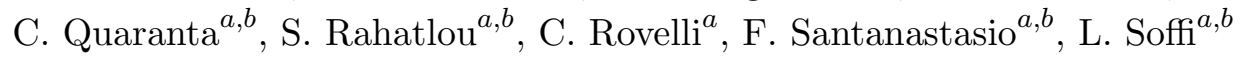

INFN Sezione di Torino ${ }^{a}$, Università di Torino ${ }^{b}$, Torino, Italy, Università del Piemonte Orientale ${ }^{c}$, Novara, Italy

N. Amapane ${ }^{a, b}$, R. Arcidiacono ${ }^{a, c}$, S. Argiro ${ }^{a, b}$, M. Arneodo $^{a, c}$, N. Bartosik ${ }^{a}$, R. Bellan $^{a, b}$, A. Bellora, C. Biino ${ }^{a}$, A. Cappati ${ }^{a, b}$, N. Cartiglia ${ }^{a}$, S. Cometti $^{a},{\text { M. } \text { Costa }^{a}, b}^{a}$, R. Covarelli $^{a, b}$, N. Demaria ${ }^{a}$, B. Kiani ${ }^{a, b}$, C. Mariotti $^{a}$, S. Maselli ${ }^{a}$, E. Migliore ${ }^{a, b}$, V. Monaco ${ }^{a, b}$, E. Monteil $^{a, b}$, M. Monteno ${ }^{a}$, M.M. Obertino ${ }^{a, b}$, G. Ortona ${ }^{a, b}$, L. Pacher $^{a, b}$, N. Pastrone ${ }^{a}$, M. Pelliccioni $^{a}$, G.L. Pinna Angioni ${ }^{a, b}$, A. Romero ${ }^{a, b}$, M. Ruspa $^{a, c}$, R. Salvatico $^{a, b}$, V. Sola $^{a}$, A. Solano ${ }^{a, b}$, D. Soldi ${ }^{a, b}$, A. Staiano ${ }^{a}$

INFN Sezione di Trieste ${ }^{a}$, Università di Trieste ${ }^{b}$, Trieste, Italy

S. Belforte ${ }^{a}$, V. Candelise ${ }^{a, b}$, M. Casarsa ${ }^{a}$, F. Cossutti ${ }^{a}$, A. Da Rold ${ }^{a, b}$, G. Della Ricca ${ }^{a, b}$, F. Vazzoler ${ }^{a, b}$, A. Zanetti ${ }^{a}$

Kyungpook National University, Daegu, Korea

B. Kim, D.H. Kim, G.N. Kim, J. Lee, S.W. Lee, C.S. Moon, Y.D. Oh, S.I. Pak, S. Sekmen, D.C. Son, Y.C. Yang

Chonnam National University, Institute for Universe and Elementary Particles, Kwangju, Korea

H. Kim, D.H. Moon, G. Oh

Hanyang University, Seoul, Korea

B. Francois, T.J. Kim, J. Park

Korea University, Seoul, Korea

S. Cho, S. Choi, Y. Go, D. Gyun, S. Ha, B. Hong, K. Lee, K.S. Lee, J. Lim, J. Park, S.K. Park, Y. Roh, J. Yoo

Kyung Hee University, Department of Physics

J. Goh 
Sejong University, Seoul, Korea

H.S. Kim

Seoul National University, Seoul, Korea

J. Almond, J.H. Bhyun, J. Choi, S. Jeon, J. Kim, J.S. Kim, H. Lee, K. Lee, S. Lee, K. Nam, M. Oh, S.B. Oh, B.C. Radburn-Smith, U.K. Yang, H.D. Yoo, I. Yoon, G.B. Yu

University of Seoul, Seoul, Korea

D. Jeon, H. Kim, J.H. Kim, J.S.H. Lee, I.C. Park, I.J Watson

Sungkyunkwan University, Suwon, Korea

Y. Choi, C. Hwang, Y. Jeong, J. Lee, Y. Lee, I. Yu

Riga Technical University, Riga, Latvia

V. Veckalns ${ }^{33}$

Vilnius University, Vilnius, Lithuania

V. Dudenas, A. Juodagalvis, G. Tamulaitis, J. Vaitkus

National Centre for Particle Physics, Universiti Malaya, Kuala Lumpur, Malaysia

Z.A. Ibrahim, F. Mohamad Idris ${ }^{34}$, W.A.T. Wan Abdullah, M.N. Yusli, Z. Zolkapli

Universidad de Sonora (UNISON), Hermosillo, Mexico

J.F. Benitez, A. Castaneda Hernandez, J.A. Murillo Quijada, L. Valencia Palomo

Centro de Investigacion y de Estudios Avanzados del IPN, Mexico City, Mexico

H. Castilla-Valdez, E. De La Cruz-Burelo, I. Heredia-De La Cruz ${ }^{35}$, R. Lopez-Fernandez,

A. Sanchez-Hernandez

Universidad Iberoamericana, Mexico City, Mexico

S. Carrillo Moreno, C. Oropeza Barrera, M. Ramirez-Garcia, F. Vazquez Valencia

Benemerita Universidad Autonoma de Puebla, Puebla, Mexico

J. Eysermans, I. Pedraza, H.A. Salazar Ibarguen, C. Uribe Estrada

Universidad Autónoma de San Luis Potosí, San Luis Potosí, Mexico

A. Morelos Pineda

University of Montenegro, Podgorica, Montenegro

J. Mijuskovic, N. Raicevic

University of Auckland, Auckland, New Zealand

D. Krofcheck

University of Canterbury, Christchurch, New Zealand

S. Bheesette, P.H. Butler

National Centre for Physics, Quaid-I-Azam University, Islamabad, Pakistan

A. Ahmad, M. Ahmad, Q. Hassan, H.R. Hoorani, W.A. Khan, M.A. Shah, M. Shoaib, M. Waqas 
AGH University of Science and Technology Faculty of Computer Science, Electronics and Telecommunications, Krakow, Poland

V. Avati, L. Grzanka, M. Malawski

National Centre for Nuclear Research, Swierk, Poland

H. Bialkowska, M. Bluj, B. Boimska, M. Górski, M. Kazana, M. Szleper, P. Zalewski

Institute of Experimental Physics, Faculty of Physics, University of Warsaw, Warsaw, Poland

K. Bunkowski, A. Byszuk ${ }^{36}$, K. Doroba, A. Kalinowski, M. Konecki, J. Krolikowski, M. Misiura, M. Olszewski, M. Walczak

Laboratório de Instrumentação e Física Experimental de Partículas, Lisboa, Portugal

M. Araujo, P. Bargassa, D. Bastos, A. Di Francesco, P. Faccioli, B. Galinhas, M. Gallinaro, J. Hollar, N. Leonardo, J. Seixas, K. Shchelina, G. Strong, O. Toldaiev, J. Varela

Joint Institute for Nuclear Research, Dubna, Russia

V. Alexakhin, M. Gavrilenko, A. Golunov, I. Golutvin, N. Gorbounov, I. Gorbunov, V. Karjavine, V. Korenkov, A. Lanev, A. Malakhov, V. Matveev ${ }^{37,38}$, P. Moisenz, V. Palichik, V. Perelygin, M. Savina, S. Shmatov, S. Shulha, N. Voytishin, B.S. Yuldashev ${ }^{39}$, A. Zarubin

Petersburg Nuclear Physics Institute, Gatchina (St. Petersburg), Russia

L. Chtchipounov, V. Golovtcov, Y. Ivanov, V. Kim ${ }^{40}$, E. Kuznetsova ${ }^{41}$, P. Levchenko, V. Murzin, V. Oreshkin, I. Smirnov, D. Sosnov, V. Sulimov, L. Uvarov, A. Vorobyev

Institute for Nuclear Research, Moscow, Russia

Yu. Andreev, A. Dermenev, S. Gninenko, N. Golubev, A. Karneyeu, M. Kirsanov, N. Krasnikov, A. Pashenkov, D. Tlisov, A. Toropin

Institute for Theoretical and Experimental Physics named by A.I. Alikhanov of NRC 'Kurchatov Institute', Moscow, Russia

V. Epshteyn, V. Gavrilov, N. Lychkovskaya, A. Nikitenko ${ }^{42}$, V. Popov, I. Pozdnyakov, G. Safronov, A. Spiridonov, A. Stepennov, M. Toms, E. Vlasov, A. Zhokin

Moscow Institute of Physics and Technology, Moscow, Russia

T. Aushev

National Research Nuclear University 'Moscow Engineering Physics Institute' (MEPhI), Moscow, Russia

M. Chadeeva ${ }^{43}$, P. Parygin, D. Philippov, E. Popova, V. Rusinov

P.N. Lebedev Physical Institute, Moscow, Russia

V. Andreev, M. Azarkin, I. Dremin, M. Kirakosyan, A. Terkulov

Skobeltsyn Institute of Nuclear Physics, Lomonosov Moscow State University, Moscow, Russia

A. Baskakov, A. Belyaev, E. Boos, V. Bunichev, M. Dubinin ${ }^{44}$, L. Dudko, A. Ershov, V. Klyukhin, O. Kodolova, I. Lokhtin, S. Obraztsov, M. Perfilov, V. Savrin 
Novosibirsk State University (NSU), Novosibirsk, Russia

A. Barnyakov ${ }^{45}$, V. Blinov ${ }^{45}$, T. Dimova ${ }^{45}$, L. Kardapoltsev ${ }^{45}$, Y. Skovpen ${ }^{45}$

Institute for High Energy Physics of National Research Centre 'Kurchatov Institute', Protvino, Russia

I. Azhgirey, I. Bayshev, S. Bitioukov, V. Kachanov, D. Konstantinov, P. Mandrik, V. Petrov, R. Ryutin, S. Slabospitskii, A. Sobol, S. Troshin, N. Tyurin, A. Uzunian, A. Volkov

National Research Tomsk Polytechnic University, Tomsk, Russia

A. Babaev, A. Iuzhakov, V. Okhotnikov

Tomsk State University, Tomsk, Russia

V. Borchsh, V. Ivanchenko, E. Tcherniaev

University of Belgrade: Faculty of Physics and VINCA Institute of Nuclear Sciences

P. Adzic ${ }^{46}$, P. Cirkovic, D. Devetak, M. Dordevic, P. Milenovic, J. Milosevic, M. Stojanovic

Centro de Investigaciones Energéticas Medioambientales y Tecnológicas (CIEMAT), Madrid, Spain

M. Aguilar-Benitez, J. Alcaraz Maestre, A. Álvarez Fernández, I. Bachiller, M. Barrio Luna, J.A. Brochero Cifuentes, C.A. Carrillo Montoya, M. Cepeda, M. Cerrada, N. Colino, B. De La Cruz, A. Delgado Peris, C. Fernandez Bedoya, J.P. Fernández Ramos, J. Flix, M.C. Fouz, O. Gonzalez Lopez, S. Goy Lopez, J.M. Hernandez, M.I. Josa, D. Moran, Á. Navarro Tobar, A. Pérez-Calero Yzquierdo, J. Puerta Pelayo, I. Redondo, L. Romero, S. Sánchez Navas, M.S. Soares, A. Triossi, C. Willmott

Universidad Autónoma de Madrid, Madrid, Spain

C. Albajar, J.F. de Trocóniz, R. Reyes-Almanza

Universidad de Oviedo, Instituto Universitario de Ciencias y Tecnologías Espaciales de Asturias (ICTEA), Oviedo, Spain

B. Alvarez Gonzalez, J. Cuevas, C. Erice, J. Fernandez Menendez, S. Folgueras, I. Gonzalez Caballero, J.R. González Fernández, E. Palencia Cortezon, V. Rodríguez Bouza, S. Sanchez Cruz

Instituto de Física de Cantabria (IFCA), CSIC-Universidad de Cantabria, Santander, Spain

I.J. Cabrillo, A. Calderon, B. Chazin Quero, J. Duarte Campderros, M. Fernandez, P.J. Fernández Manteca, A. García Alonso, G. Gomez, C. Martinez Rivero, P. Martinez Ruiz del Arbol, F. Matorras, J. Piedra Gomez, C. Prieels, T. Rodrigo, A. Ruiz-Jimeno, L. Russo $^{47}$, L. Scodellaro, N. Trevisani, I. Vila, J.M. Vizan Garcia

University of Colombo, Colombo, Sri Lanka

K. Malagalage

University of Ruhuna, Department of Physics, Matara, Sri Lanka

W.G.D. Dharmaratna, N. Wickramage 


\section{CERN, European Organization for Nuclear Research, Geneva, Switzerland}

D. Abbaneo, B. Akgun, E. Auffray, G. Auzinger, J. Baechler, P. Baillon, A.H. Ball, D. Barney, J. Bendavid, M. Bianco, A. Bocci, P. Bortignon, E. Bossini, C. Botta, E. Brondolin, T. Camporesi, A. Caratelli, G. Cerminara, E. Chapon, G. Cucciati, D. d'Enterria, A. Dabrowski, N. Daci, V. Daponte, A. David, O. Davignon, A. De Roeck, N. Deelen, M. Deile, M. Dobson, M. Dünser, N. Dupont, A. Elliott-Peisert, N. Emriskova, F. Fallavollita ${ }^{48}$, D. Fasanella, S. Fiorendi, G. Franzoni, J. Fulcher, W. Funk, S. Giani, D. Gigi, A. Gilbert, K. Gill, F. Glege, M. Gruchala, M. Guilbaud, D. Gulhan, J. Hegeman, C. Heidegger, Y. Iiyama, V. Innocente, P. Janot, O. Karacheban ${ }^{20}$, J. Kaspar, J. Kieseler, M. Krammer ${ }^{1}$, N. Kratochwil, C. Lange, P. Lecoq, C. Lourenço, L. Malgeri, M. Mannelli, A. Massironi, F. Meijers, J.A. Merlin, S. Mersi, E. Meschi, F. Moortgat, M. Mulders, J. Ngadiuba, J. Niedziela, S. Nourbakhsh, S. Orfanelli, L. Orsini, F. Pantaleo ${ }^{17}$, L. Pape, E. Perez, M. Peruzzi, A. Petrilli, G. Petrucciani, A. Pfeiffer, M. Pierini, F.M. Pitters, D. Rabady, A. Racz, M. Rieger, M. Rovere, H. Sakulin, C. Schäfer, C. Schwick, M. Selvaggi, A. Sharma, P. Silva, W. Snoeys, P. Sphicas ${ }^{49}$, J. Steggemann, S. Summers, V.R. Tavolaro, D. Treille, A. Tsirou, G.P. Van Onsem, A. Vartak, M. Verzetti, W.D. Zeuner

\section{Paul Scherrer Institut, Villigen, Switzerland}

L. Caminada ${ }^{50}$, K. Deiters, W. Erdmann, R. Horisberger, Q. Ingram, H.C. Kaestli, D. Kotlinski, U. Langenegger, T. Rohe, S.A. Wiederkehr

ETH Zurich - Institute for Particle Physics and Astrophysics (IPA), Zurich, Switzerland

M. Backhaus, P. Berger, N. Chernyavskaya, G. Dissertori, M. Dittmar, M. Donegà, C. Dorfer, T.A. Gómez Espinosa, C. Grab, D. Hits, T. Klijnsma, W. Lustermann, R.A. Manzoni, M. Marionneau, M.T. Meinhard, F. Micheli, P. Musella, F. Nessi-Tedaldi, F. Pauss, G. Perrin, L. Perrozzi, S. Pigazzini, M.G. Ratti, M. Reichmann, C. Reissel, T. Reitenspiess, D. Ruini, D.A. Sanz Becerra, M. Schönenberger, L. Shchutska, M.L. Vesterbacka Olsson, R. Wallny, D.H. Zhu

\section{Universität Zürich, Zurich, Switzerland}

T.K. Aarrestad, C. Amsler ${ }^{51}$, D. Brzhechko, M.F. Canelli, A. De Cosa, R. Del Burgo, S. Donato, B. Kilminster, S. Leontsinis, V.M. Mikuni, I. Neutelings, G. Rauco, P. Robmann, D. Salerno, K. Schweiger, C. Seitz, Y. Takahashi, S. Wertz, A. Zucchetta

National Central University, Chung-Li, Taiwan

C. Adloff ${ }^{52}$, T.H. Doan, C.M. Kuo, W. Lin, A. Roy, S.S. Yu

National Taiwan University (NTU), Taipei, Taiwan

P. Chang, Y. Chao, K.F. Chen, P.H. Chen, W.-S. Hou, Y.y. Li, R.-S. Lu, E. Paganis, A. Psallidas, A. Steen

Chulalongkorn University, Faculty of Science, Department of Physics, Bangkok, Thailand

B. Asavapibhop, C. Asawatangtrakuldee, N. Srimanobhas, N. Suwonjandee 
Çukurova University, Physics Department, Science and Art Faculty, Adana, Turkey

A. Bat, F. Boran, A. Celik ${ }^{53}$, S. Cerci ${ }^{54}$, S. Damarseckin ${ }^{55}$, Z.S. Demiroglu, F. Dolek, C. Dozen ${ }^{56}$, I. Dumanoglu, G. Gokbulut, EmineGurpinar Guler ${ }^{57}$, Y. Guler, I. Hos ${ }^{58}$, C. Isik, E.E. Kangal ${ }^{59}$, O. Kara, A. Kayis Topaksu, U. Kiminsu, M. Oglakci, G. Onengut, K. Ozdemir $^{60}$, S. Ozturk ${ }^{61}$, A.E. Simsek, D. Sunar Cerci ${ }^{54}$, U.G. Tok, S. Turkcapar, I.S. Zorbakir, C. Zorbilmez

Middle East Technical University, Physics Department, Ankara, Turkey B. Isildak ${ }^{62}$, G. Karapinar ${ }^{63}$, M. Yalvac

Bogazici University, Istanbul, Turkey

I.O. Atakisi, E. Gülmez, M. Kaya ${ }^{64}$, O. Kaya ${ }^{65}$, Ö. Özçelik, S. Tekten, E.A. Yetkin ${ }^{66}$

Istanbul Technical University, Istanbul, Turkey

A. Cakir, Y. Komurcu, S. Sen ${ }^{67}$

Istanbul University, Istanbul, Turkey

B. Kaynak, S. Ozkorucuklu

Institute for Scintillation Materials of National Academy of Science of Ukraine, Kharkov, Ukraine

B. Grynyov

National Scientific Center, Kharkov Institute of Physics and Technology, Kharkov, Ukraine

L. Levchuk

University of Bristol, Bristol, United Kingdom

F. Ball, E. Bhal, S. Bologna, J.J. Brooke, D. Burns ${ }^{68}$, E. Clement, D. Cussans, H. Flacher, J. Goldstein, G.P. Heath, H.F. Heath, L. Kreczko, S. Paramesvaran, B. Penning, T. Sakuma, S. Seif El Nasr-Storey, V.J. Smith, J. Taylor, A. Titterton

Rutherford Appleton Laboratory, Didcot, United Kingdom

K.W. Bell, A. Belyaev ${ }^{69}$, C. Brew, R.M. Brown, D. Cieri, D.J.A. Cockerill, J.A. Coughlan, K. Harder, S. Harper, J. Linacre, K. Manolopoulos, D.M. Newbold, E. Olaiya, D. Petyt, T. Reis, T. Schuh, C.H. Shepherd-Themistocleous, A. Thea, I.R. Tomalin, T. Williams, W.J. Womersley

\section{Imperial College, London, United Kingdom}

R. Bainbridge, P. Bloch, J. Borg, S. Breeze, O. Buchmuller, A. Bundock, GurpreetSingh CHAHAL ${ }^{70}$, D. Colling, P. Dauncey, G. Davies, M. Della Negra, R. Di Maria, P. Everaerts, G. Hall, G. Iles, T. James, M. Komm, C. Laner, L. Lyons, A.-M. Magnan, S. Malik, A. Martelli, V. Milosevic, J. Nash ${ }^{71}$, V. Palladino, M. Pesaresi, D.M. Raymond, A. Richards, A. Rose, E. Scott, C. Seez, A. Shtipliyski, M. Stoye, T. Strebler, A. Tapper, K. Uchida, T. Virdee ${ }^{17}$, N. Wardle, D. Winterbottom, J. Wright, A.G. Zecchinelli, S.C. Zenz 
Brunel University, Uxbridge, United Kingdom

J.E. Cole, P.R. Hobson, A. Khan, P. Kyberd, C.K. Mackay, A. Morton, I.D. Reid, L. Teodorescu, S. Zahid

Baylor University, Waco, U.S.A.

K. Call, B. Caraway, J. Dittmann, K. Hatakeyama, C. Madrid, B. McMaster, N. Pastika, C. Smith

Catholic University of America, Washington, DC, U.S.A.

R. Bartek, A. Dominguez, R. Uniyal, A.M. Vargas Hernandez

The University of Alabama, Tuscaloosa, U.S.A.

A. Buccilli, S.I. Cooper, C. Henderson, P. Rumerio, C. West

Boston University, Boston, U.S.A.

D. Arcaro, Z. Demiragli, D. Gastler, D. Pinna, C. Richardson, J. Rohlf, D. Sperka, I. Suarez, L. Sulak, D. Zou

Brown University, Providence, U.S.A.

G. Benelli, B. Burkle, X. Coubez ${ }^{18}$, D. Cutts, Y.t. Duh, M. Hadley, J. Hakala, U. Heintz, J.M. $\operatorname{Hogan}^{72}$, K.H.M. Kwok, E. Laird, G. Landsberg, J. Lee, Z. Mao, M. Narain, S. Sagir ${ }^{73}$, R. Syarif, E. Usai, D. Yu, W. Zhang

University of California, Davis, Davis, U.S.A.

R. Band, C. Brainerd, R. Breedon, M. Calderon De La Barca Sanchez, M. Chertok, J. Conway, R. Conway, P.T. Cox, R. Erbacher, C. Flores, G. Funk, F. Jensen, W. Ko, O. Kukral, R. Lander, M. Mulhearn, D. Pellett, J. Pilot, M. Shi, D. Taylor, K. Tos, M. Tripathi, Z. Wang, F. Zhang

University of California, Los Angeles, U.S.A.

M. Bachtis, C. Bravo, R. Cousins, A. Dasgupta, A. Florent, J. Hauser, M. Ignatenko, N. Mccoll, W.A. Nash, S. Regnard, D. Saltzberg, C. Schnaible, B. Stone, V. Valuev

University of California, Riverside, Riverside, U.S.A.

K. Burt, Y. Chen, R. Clare, J.W. Gary, S.M.A. Ghiasi Shirazi, G. Hanson, G. Karapostoli, E. Kennedy, O.R. Long, M. Olmedo Negrete, M.I. Paneva, W. Si, L. Wang, S. Wimpenny, B.R. Yates, Y. Zhang

University of California, San Diego, La Jolla, U.S.A.

J.G. Branson, P. Chang, S. Cittolin, M. Derdzinski, R. Gerosa, D. Gilbert, B. Hashemi, D. Klein, V. Krutelyov, J. Letts, M. Masciovecchio, S. May, S. Padhi, M. Pieri, V. Sharma, M. Tadel, F. Würthwein, A. Yagil, G. Zevi Della Porta

University of California, Santa Barbara - Department of Physics, Santa Barbara, U.S.A.

N. Amin, R. Bhandari, C. Campagnari, M. Citron, V. Dutta, M. Franco Sevilla, L. Gouskos, J. Incandela, B. Marsh, H. Mei, A. Ovcharova, H. Qu, J. Richman, U. Sarica, D. Stuart, S. Wang 
California Institute of Technology, Pasadena, U.S.A.

D. Anderson, A. Bornheim, O. Cerri, I. Dutta, J.M. Lawhorn, N. Lu, J. Mao, H.B. Newman, T.Q. Nguyen, J. Pata, M. Spiropulu, J.R. Vlimant, S. Xie, Z. Zhang, R.Y. Zhu

Carnegie Mellon University, Pittsburgh, U.S.A.

M.B. Andrews, T. Ferguson, T. Mudholkar, M. Paulini, M. Sun, I. Vorobiev, M. Weinberg

University of Colorado Boulder, Boulder, U.S.A.

J.P. Cumalat, W.T. Ford, A. Johnson, E. MacDonald, T. Mulholland, R. Patel, A. Perloff, K. Stenson, K.A. Ulmer, S.R. Wagner

Cornell University, Ithaca, U.S.A.

J. Alexander, J. Chaves, Y. Cheng, J. Chu, A. Datta, A. Frankenthal, K. Mcdermott, J.R. Patterson, D. Quach, A. Rinkevicius ${ }^{74}$, A. Ryd, S.M. Tan, Z. Tao, J. Thom, P. Wittich, M. Zientek

\section{Fermi National Accelerator Laboratory, Batavia, U.S.A.}

S. Abdullin, M. Albrow, M. Alyari, G. Apollinari, A. Apresyan, A. Apyan, S. Banerjee, L.A.T. Bauerdick, A. Beretvas, D. Berry, J. Berryhill, P.C. Bhat, K. Burkett, J.N. Butler, A. Canepa, G.B. Cerati, H.W.K. Cheung, F. Chlebana, M. Cremonesi, J. Duarte, V.D. Elvira, J. Freeman, Z. Gecse, E. Gottschalk, L. Gray, D. Green, S. Grünendahl, O. Gutsche, AllisonReinsvold Hall, J. Hanlon, R.M. Harris, S. Hasegawa, R. Heller, J. Hirschauer, B. Jayatilaka, S. Jindariani, M. Johnson, U. Joshi, B. Klima, M.J. Kortelainen, B. Kreis, S. Lammel, J. Lewis, D. Lincoln, R. Lipton, M. Liu, T. Liu, J. Lykken, K. Maeshima, J.M. Marraffino, D. Mason, P. McBride, P. Merkel, S. Mrenna, S. Nahn, V. O'Dell, V. Papadimitriou, K. Pedro, C. Pena, G. Rakness, F. Ravera, L. Ristori, B. Schneider, E. Sexton-Kennedy, N. Smith, A. Soha, W.J. Spalding, L. Spiegel, S. Stoynev, J. Strait, N. Strobbe, L. Taylor, S. Tkaczyk, N.V. Tran, L. Uplegger, E.W. Vaandering, C. Vernieri, R. Vidal, M. Wang, H.A. Weber

\section{University of Florida, Gainesville, U.S.A.}

D. Acosta, P. Avery, D. Bourilkov, A. Brinkerhoff, L. Cadamuro, A. Carnes, V. Cherepanov, D. Curry, F. Errico, R.D. Field, S.V. Gleyzer, B.M. Joshi, M. Kim, J. Konigsberg, A. Korytov, K.H. Lo, P. Ma, K. Matchev, N. Menendez, G. Mitselmakher, D. Rosenzweig, K. Shi, J. Wang, S. Wang, X. Zuo

Florida International University, Miami, U.S.A.

Y.R. Joshi

Florida State University, Tallahassee, U.S.A.

T. Adams, A. Askew, S. Hagopian, V. Hagopian, K.F. Johnson, R. Khurana, T. Kolberg, G. Martinez, T. Perry, H. Prosper, C. Schiber, R. Yohay, J. Zhang

Florida Institute of Technology, Melbourne, U.S.A.

M.M. Baarmand, M. Hohlmann, D. Noonan, M. Rahmani, M. Saunders, F. Yumiceva 
University of Illinois at Chicago (UIC), Chicago, U.S.A.

M.R. Adams, L. Apanasevich, R.R. Betts, R. Cavanaugh, X. Chen, S. Dittmer, O. Evdokimov, C.E. Gerber, D.A. Hangal, D.J. Hofman, K. Jung, C. Mills, T. Roy, M.B. Tonjes, N. Varelas, J. Viinikainen, H. Wang, X. Wang, Z. Wu

\section{The University of Iowa, Iowa City, U.S.A.}

M. Alhusseini, B. Bilki ${ }^{57}$, W. Clarida, K. Dilsiz ${ }^{75}$, S. Durgut, R.P. Gandrajula, M. Haytmyradov, V. Khristenko, O.K. Köseyan, J.-P. Merlo, A. Mestvirishvili ${ }^{76}$, A. Moeller, J. Nachtman, H. Ogul ${ }^{77}$, Y. Onel, F. Ozok ${ }^{78}$, A. Penzo, C. Snyder, E. Tiras, J. Wetzel

Johns Hopkins University, Baltimore, U.S.A.

B. Blumenfeld, A. Cocoros, N. Eminizer, D. Fehling, L. Feng, A.V. Gritsan, W.T. Hung, P. Maksimovic, J. Roskes, M. Swartz

The University of Kansas, Lawrence, U.S.A.

C. Baldenegro Barrera, P. Baringer, A. Bean, S. Boren, J. Bowen, A. Bylinkin, T. Isidori, S. Khalil, J. King, G. Krintiras, A. Kropivnitskaya, C. Lindsey, D. Majumder, W. Mcbrayer, N. Minafra, M. Murray, C. Rogan, C. Royon, S. Sanders, E. Schmitz, J.D. Tapia Takaki, Q. Wang, J. Williams, G. Wilson

Kansas State University, Manhattan, U.S.A.

S. Duric, A. Ivanov, K. Kaadze, D. Kim, Y. Maravin, D.R. Mendis, T. Mitchell, A. Modak, A. Mohammadi

Lawrence Livermore National Laboratory, Livermore, U.S.A.

F. Rebassoo, D. Wright

University of Maryland, College Park, U.S.A.

A. Baden, O. Baron, A. Belloni, S.C. Eno, Y. Feng, N.J. Hadley, S. Jabeen, G.Y. Jeng, R.G. Kellogg, J. Kunkle, A.C. Mignerey, S. Nabili, F. Ricci-Tam, M. Seidel, Y.H. Shin, A. Skuja, S.C. Tonwar, K. Wong

\section{Massachusetts Institute of Technology, Cambridge, U.S.A.}

D. Abercrombie, B. Allen, A. Baty, R. Bi, S. Brandt, W. Busza, I.A. Cali, M. D'Alfonso,

G. Gomez Ceballos, M. Goncharov, P. Harris, D. Hsu, M. Hu, M. Klute, D. Kovalskyi, Y.-J. Lee, P.D. Luckey, B. Maier, A.C. Marini, C. Mcginn, C. Mironov, S. Narayanan, X. Niu, C. Paus, D. Rankin, C. Roland, G. Roland, Z. Shi, G.S.F. Stephans, K. Sumorok, K. Tatar, D. Velicanu, J. Wang, T.W. Wang, B. Wyslouch

University of Minnesota, Minneapolis, U.S.A.

A.C. Benvenuti ${ }^{\dagger}$, R.M. Chatterjee, A. Evans, S. Guts, P. Hansen, J. Hiltbrand, Y. Kubota, Z. Lesko, J. Mans, R. Rusack, M.A. Wadud

University of Mississippi, Oxford, U.S.A.

J.G. Acosta, S. Oliveros

University of Nebraska-Lincoln, Lincoln, U.S.A.

K. Bloom, D.R. Claes, C. Fangmeier, L. Finco, F. Golf, R. Gonzalez Suarez, R. Kamalieddin, I. Kravchenko, J.E. Siado, G.R. Snow ${ }^{\dagger}$, B. Stieger, W. Tabb 
State University of New York at Buffalo, Buffalo, U.S.A.

G. Agarwal, C. Harrington, I. Iashvili, A. Kharchilava, C. McLean, D. Nguyen, A. Parker, J. Pekkanen, S. Rappoccio, B. Roozbahani

Northeastern University, Boston, U.S.A.

G. Alverson, E. Barberis, C. Freer, Y. Haddad, A. Hortiangtham, G. Madigan, D.M. Morse,

T. Orimoto, L. Skinnari, A. Tishelman-Charny, T. Wamorkar, B. Wang, A. Wisecarver,

D. Wood

Northwestern University, Evanston, U.S.A.

S. Bhattacharya, J. Bueghly, T. Gunter, K.A. Hahn, N. Odell, M.H. Schmitt, K. Sung, M. Trovato, M. Velasco

University of Notre Dame, Notre Dame, U.S.A.

R. Bucci, N. Dev, R. Goldouzian, M. Hildreth, K. Hurtado Anampa, C. Jessop, D.J. Karmgard, K. Lannon, W. Li, N. Loukas, N. Marinelli, I. Mcalister, F. Meng, C. Mueller, Y. Musienko ${ }^{37}$, M. Planer, R. Ruchti, P. Siddireddy, G. Smith, S. Taroni, M. Wayne, A. Wightman, M. Wolf, A. Woodard

The Ohio State University, Columbus, U.S.A.

J. Alimena, B. Bylsma, L.S. Durkin, S. Flowers, B. Francis, C. Hill, W. Ji, A. Lefeld, T.Y. Ling, B.L. Winer

Princeton University, Princeton, U.S.A.

S. Cooperstein, G. Dezoort, P. Elmer, J. Hardenbrook, N. Haubrich, S. Higginbotham, A. Kalogeropoulos, S. Kwan, D. Lange, M.T. Lucchini, J. Luo, D. Marlow, K. Mei, I. Ojalvo, J. Olsen, C. Palmer, P. Piroué, J. Salfeld-Nebgen, D. Stickland, C. Tully, Z. Wang

University of Puerto Rico, Mayaguez, U.S.A.

S. Malik, S. Norberg

Purdue University, West Lafayette, U.S.A.

A. Barker, V.E. Barnes, S. Das, L. Gutay, M. Jones, A.W. Jung, A. Khatiwada, B. Mahakud, D.H. Miller, G. Negro, N. Neumeister, C.C. Peng, S. Piperov, H. Qiu, J.F. Schulte, J. Sun, F. Wang, R. Xiao, W. Xie

Purdue University Northwest, Hammond, U.S.A.

T. Cheng, J. Dolen, N. Parashar

Rice University, Houston, U.S.A.

U. Behrens, K.M. Ecklund, S. Freed, F.J.M. Geurts, M. Kilpatrick, Arun Kumar, W. Li, B.P. Padley, R. Redjimi, J. Roberts, J. Rorie, W. Shi, A.G. Stahl Leiton, Z. Tu, A. Zhang

University of Rochester, Rochester, U.S.A.

A. Bodek, P. de Barbaro, R. Demina, J.L. Dulemba, C. Fallon, T. Ferbel, M. Galanti,

A. Garcia-Bellido, O. Hindrichs, A. Khukhunaishvili, E. Ranken, P. Tan, R. Taus 
Rutgers, The State University of New Jersey, Piscataway, U.S.A.

B. Chiarito, J.P. Chou, A. Gandrakota, Y. Gershtein, E. Halkiadakis, A. Hart, M. Heindl, E. Hughes, S. Kaplan, S. Kyriacou, I. Laflotte, A. Lath, R. Montalvo, K. Nash, M. Osherson, H. Saka, S. Salur, S. Schnetzer, S. Somalwar, R. Stone, S. Thomas

University of Tennessee, Knoxville, U.S.A.

H. Acharya, A.G. Delannoy, G. Riley, S. Spanier

Texas A\&M University, College Station, U.S.A.

O. Bouhali ${ }^{79}$, M. Dalchenko, M. De Mattia, A. Delgado, S. Dildick, R. Eusebi, J. Gilmore, T. Huang, T. Kamon ${ }^{80}$, S. Luo, D. Marley, R. Mueller, D. Overton, L. Perniè, D. Rathjens,

A. Safonov

Texas Tech University, Lubbock, U.S.A.

N. Akchurin, J. Damgov, F. De Guio, S. Kunori, K. Lamichhane, S.W. Lee, T. Mengke, S. Muthumuni, T. Peltola, S. Undleeb, I. Volobouev, Z. Wang, A. Whitbeck

\section{Vanderbilt University, Nashville, U.S.A.}

S. Greene, A. Gurrola, R. Janjam, W. Johns, C. Maguire, A. Melo, H. Ni, K. Padeken, F. Romeo, P. Sheldon, S. Tuo, J. Velkovska, M. Verweij

University of Virginia, Charlottesville, U.S.A.

M.W. Arenton, P. Barria, B. Cox, G. Cummings, R. Hirosky, M. Joyce, A. Ledovskoy, C. Neu, B. Tannenwald, Y. Wang, E. Wolfe, F. Xia

Wayne State University, Detroit, U.S.A.

R. Harr, P.E. Karchin, N. Poudyal, J. Sturdy, P. Thapa

University of Wisconsin - Madison, Madison, WI, U.S.A.

T. Bose, J. Buchanan, C. Caillol, D. Carlsmith, S. Dasu, I. De Bruyn, L. Dodd, F. Fiori, C. Galloni, B. Gomber ${ }^{81}$, H. He, M. Herndon, A. Hervé, U. Hussain, P. Klabbers, A. Lanaro, A. Loeliger, K. Long, R. Loveless, J. Madhusudanan Sreekala, T. Ruggles, A. Savin, V. Sharma, W.H. Smith, D. Teague, S. Trembath-reichert, N. Woods

$\dagger$ : Deceased

1: Also at Vienna University of Technology, Vienna, Austria

2: Also at IRFU, CEA, Université Paris-Saclay, Gif-sur-Yvette, France

3: Also at Universidade Estadual de Campinas, Campinas, Brazil

4: Also at Federal University of Rio Grande do Sul, Porto Alegre, Brazil

5: Also at UFMS, Nova Andradina, Brazil

6: Also at Universidade Federal de Pelotas, Pelotas, Brazil

7: Also at Université Libre de Bruxelles, Bruxelles, Belgium

8: Also at University of Chinese Academy of Sciences, Beijing, China

9: Also at Institute for Theoretical and Experimental Physics named by A.I. Alikhanov of NRC 'Kurchatov Institute', Moscow, Russia

10: Also at Joint Institute for Nuclear Research, Dubna, Russia

11: Now at British University in Egypt, Cairo, Egypt

12: Now at Ain Shams University, Cairo, Egypt 
13: Also at Purdue University, West Lafayette, U.S.A.

14: Also at Université de Haute Alsace, Mulhouse, France

15: Also at Tbilisi State University, Tbilisi, Georgia

16: Also at Erzincan Binali Yildirim University, Erzincan, Turkey

17: Also at CERN, European Organization for Nuclear Research, Geneva, Switzerland

18: Also at RWTH Aachen University, III. Physikalisches Institut A, Aachen, Germany

19: Also at University of Hamburg, Hamburg, Germany

20: Also at Brandenburg University of Technology, Cottbus, Germany

21: Also at Institute of Physics, University of Debrecen, Debrecen, Hungary, Debrecen, Hungary

22: Also at Institute of Nuclear Research ATOMKI, Debrecen, Hungary

23: Also at MTA-ELTE Lendület CMS Particle and Nuclear Physics Group, Eötvös Loránd University, Budapest, Hungary, Budapest, Hungary

24: Also at IIT Bhubaneswar, Bhubaneswar, India, Bhubaneswar, India

25: Also at Institute of Physics, Bhubaneswar, India

26: Also at Shoolini University, Solan, India

27: Also at University of Visva-Bharati, Santiniketan, India

28: Also at Isfahan University of Technology, Isfahan, Iran

29: Now at INFN Sezione di Bari ${ }^{a}$, Università di Bari ${ }^{b}$, Politecnico di Bari ${ }^{c}$, Bari, Italy

30: Also at Italian National Agency for New Technologies, Energy and Sustainable Economic Development, Bologna, Italy

31: Also at Centro Siciliano di Fisica Nucleare e di Struttura Della Materia, Catania, Italy

32: Also at Scuola Normale e Sezione dell'INFN, Pisa, Italy

33: Also at Riga Technical University, Riga, Latvia, Riga, Latvia

34: Also at Malaysian Nuclear Agency, MOSTI, Kajang, Malaysia

35: Also at Consejo Nacional de Ciencia y Tecnología, Mexico City, Mexico

36: Also at Warsaw University of Technology, Institute of Electronic Systems, Warsaw, Poland

37: Also at Institute for Nuclear Research, Moscow, Russia

38: Now at National Research Nuclear University 'Moscow Engineering Physics Institute' (MEPhI), Moscow, Russia

39: Also at Institute of Nuclear Physics of the Uzbekistan Academy of Sciences, Tashkent, Uzbekistan

40: Also at St. Petersburg State Polytechnical University, St. Petersburg, Russia

41: Also at University of Florida, Gainesville, U.S.A.

42: Also at Imperial College, London, United Kingdom

43: Also at P.N. Lebedev Physical Institute, Moscow, Russia

44: Also at California Institute of Technology, Pasadena, U.S.A.

45: Also at Budker Institute of Nuclear Physics, Novosibirsk, Russia

46: Also at Faculty of Physics, University of Belgrade, Belgrade, Serbia

47: Also at Università degli Studi di Siena, Siena, Italy

48: Also at INFN Sezione di Pavia ${ }^{a}$, Università di Pavia ${ }^{b}$, Pavia, Italy, Pavia, Italy

49: Also at National and Kapodistrian University of Athens, Athens, Greece

50: Also at Universität Zürich, Zurich, Switzerland

51: Also at Stefan Meyer Institute for Subatomic Physics, Vienna, Austria, Vienna, Austria

52: Also at Laboratoire d'Annecy-le-Vieux de Physique des Particules, IN2P3-CNRS, Annecyle-Vieux, France

53: Also at Burdur Mehmet Akif Ersoy University, BURDUR, Turkey

54: Also at Adiyaman University, Adiyaman, Turkey

55: Also at Şrnak University, Sirnak, Turkey 
56: Also at Tsinghua University, Beijing, China

57: Also at Beykent University, Istanbul, Turkey, Istanbul, Turkey

58: Also at Istanbul Aydin University, Istanbul, Turkey

59: Also at Mersin University, Mersin, Turkey

60: Also at Piri Reis University, Istanbul, Turkey

61: Also at Gaziosmanpasa University, Tokat, Turkey

62: Also at Ozyegin University, Istanbul, Turkey

63: Also at Izmir Institute of Technology, Izmir, Turkey

64: Also at Marmara University, Istanbul, Turkey

65: Also at Kafkas University, Kars, Turkey

66: Also at Istanbul Bilgi University, Istanbul, Turkey

67: Also at Hacettepe University, Ankara, Turkey

68: Also at Vrije Universiteit Brussel, Brussel, Belgium

69: Also at School of Physics and Astronomy, University of Southampton, Southampton, United Kingdom

70: Also at IPPP Durham University, Durham, United Kingdom

71: Also at Monash University, Faculty of Science, Clayton, Australia

72: Also at Bethel University, St. Paul, Minneapolis, U.S.A., St. Paul, U.S.A.

73: Also at Karamanoğlu Mehmetbey University, Karaman, Turkey

74: Also at Vilnius University, Vilnius, Lithuania

75: Also at Bingol University, Bingol, Turkey

76: Also at Georgian Technical University, Tbilisi, Georgia

77: Also at Sinop University, Sinop, Turkey

78: Also at Mimar Sinan University, Istanbul, Istanbul, Turkey

79: Also at Texas A\&M University at Qatar, Doha, Qatar

80: Also at Kyungpook National University, Daegu, Korea, Daegu, Korea

81: Also at University of Hyderabad, Hyderabad, India 